\title{
Untersuchungen zu kubischen metaplektischen Formen
}

\author{
Dissertation \\ zur Erlangung des Doktorgrades \\ der Mathematisch-Naturwissenschaftlichen Fakultäten \\ der Georg-August-Universität zu Göttingen
}

vorgelegt von

Leonhard Möhring

aus Göttingen

Göttingen 2003 
D 7

Referent: Professor S. J. Patterson

Korreferent: Professor U. Stuhler

Tag der mündlichen Prüfung: 4. Dezember 2003 


\section{Inhaltsverzeichnis}

1 Einleitung 11

2 Gruppentheorie und automorphe Formen $\quad 13$

2.1 Die Automorphe Gruppe . . . . . . . . . . . . . . . . . . . 13

2.2 Automorphe Formen auf $\operatorname{PSL}(2, \mathrm{Z}[\omega]) \ldots \ldots \ldots \ldots \ldots$

2.3 Die Metaplektische Gruppe . . . . . . . . . . . . . . . . . . . . . 21

2.4 Automorphe Formen auf $\tilde{\Gamma} \ldots \ldots \ldots \ldots \ldots$

3 Eigenwerte von $\Delta$ auf der Bianchigruppe zu $D=-3 \quad 35$

3.1 Numerische Bestimmung der Eigenfunktionen . . . . . . . . . . . . 36

3.2 Ausführung des Algorithmus . . . . . . . . . . . . . . . . . . . . . 39

4 Fourierkoeffizienten metaplektischer Formen $\quad 47$

4.1 Erste Vereinfachung der Fourierdarstellung . . . . . . . . . . . . . . . . . 48

4.2 Zweite Vereinfachung der Fourierdarstellung . . . . . . . . . . . . . 50

4.3 Dritte Vereinfachung der Fourierdarstellung . . . . . . . . . . . . . 52

4.4 Ausführung der Kollokation . . . . . . . . . . . . . . . . . . 54

5 Bestimmung der metaplektischen Formen $\quad 59$

5.1 Numerische Bestimmung . . . . . . . . . . . . . . . . . . . 59

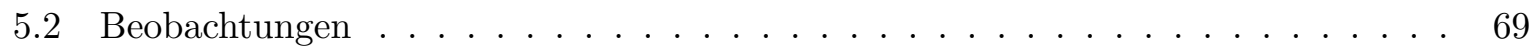

6 Numerischer Anhang $\quad 75$

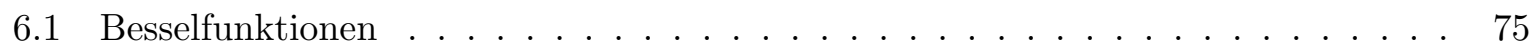


6.2 Integral der Besselfunktion $\ldots \ldots \ldots \ldots \ldots \ldots$

6.3 Kubisches Restsymbol . . . . . . . . . . . . . . . . . . 77

Bibliographie................................... 79 


\section{Abbildungsverzeichnis}

2.1 Der Fundamentalbereich $\mathcal{F}_{3}$ von $\Gamma=\operatorname{PSL}(2, \mathrm{Z}[\omega]) \ldots \ldots \ldots \ldots \ldots$

2.2 (a) Die Bereiche $\mathcal{F}_{3}, R \mathcal{F}_{3}, R^{2} \mathcal{F}_{3}$, und $2.2\left(\right.$ b) der Bereich $R S \mathcal{F}_{3} \ldots \ldots \ldots$

2.3 Der Fundamentalbereich $\widetilde{\mathcal{F}}_{3}=\bigcup_{i} \gamma_{i} \mathcal{F}_{3} \ldots \ldots \ldots \ldots \ldots$

2.4 Die Numerierung der Bereiche $13-18$ und $22-27 \quad \ldots \ldots \ldots$. . . . . . . . . . 24

2.5 (a) Projektion der zu $\infty$ gehörenden Bereiche . . . . . . . . . . . . 25

2.5 (b), (c) Projektion der zu $-\omega_{6}$ und $\omega_{6}$ gehörenden Bereiche $\ldots \ldots \ldots \ldots$

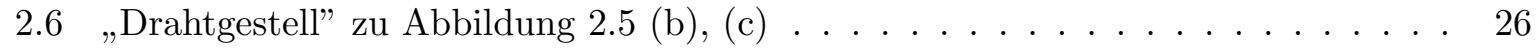

2.7 (a), (b) Die 27 Bereiche mit ihren Nachbarn . . . . . . . . . . . . . . . . 27

2.8 Der "Nachbarschaftsgraph" von $\tilde{\Gamma} \backslash \mathbb{H}^{3} \ldots \ldots \ldots \ldots \ldots \ldots \ldots \ldots$

2.9 (a) Alternative der zu $\infty$ gehörenden Bereiche . . . . . . . . . . . . . . . 29

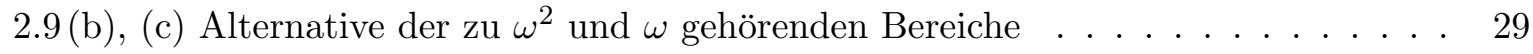

$3.1 \quad$ Die Eigenwerte $R_{1}^{-}-R_{8}^{-} \quad \ldots \ldots \ldots \ldots \ldots \ldots \ldots$

3.2 Einfluß von $N$ auf die Eigenwertsuche $\ldots \ldots \ldots \ldots \ldots$

3.3 Einfluß von $\nu$ auf die Eigenwertsuche . . . . . . . . . . . . . . . . . 40

3.4 Die kleinsten Eigenwerte der geraden Eigenfunktionen . . . . . . . . . . . . . . 41

3.5 Die Eigenwerte $R_{9}^{-}-R_{19}^{-} \ldots \ldots \ldots \ldots \ldots \ldots \ldots \ldots \ldots$

3.6 Die Eigenwerte $R_{4}^{+}-R_{7}^{+} \quad \ldots \ldots \ldots \ldots \ldots \ldots \ldots \ldots \ldots \ldots$

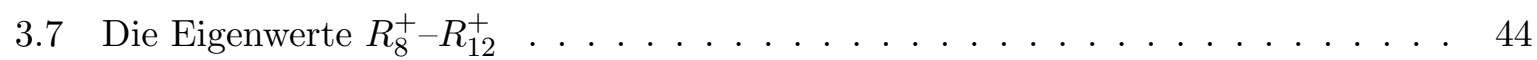

3.8 Die Eigenwerte $R_{13}^{+}-R_{22}^{+} \ldots \ldots \ldots \ldots \ldots \ldots \ldots \ldots \ldots$

5.1 Eigenwertsuche für gerade Eigenfunktionen $(\mathrm{R}$ von $2.5-7) \ldots \ldots \ldots \ldots$

5.2 Eigenwertsuche für ungerade Eigenfunktionen $(\mathrm{R}$ von $2-6) \ldots \ldots$. . . . . . 62 
5.3 Eigenwertsuche für gerade Eigenfunktionen $(\mathrm{R}$ von $7-8.3) \ldots \ldots \ldots$

5.4 Eigenwertsuche für ungerade Eigenfunktionen $(\mathrm{R}$ von $6-8.8) \ldots \ldots$. . . . . 63

5.5 Eigenwertsuche für gerade Eigenfunktionen $(\mathrm{R}$ von $8.3-9) \ldots \ldots$. . . . . . 63

5.6 Eigenwertsuche für gerade Eigenfunktionen $(\mathrm{R}$ von $9-9.8) \ldots \ldots$. . . . . 64

5.7 Plot der Argumente bei $R=R_{12}^{+}$für quadratfreie $n$ und $3 \nmid n \ldots \ldots$. . . . . 73

5.8 Plot der Argumente bei $R=R_{8}^{-}$für quadratfreie $n$ und $3 \nmid n \ldots \ldots \ldots$ 


\section{Tabellenverzeichnis}

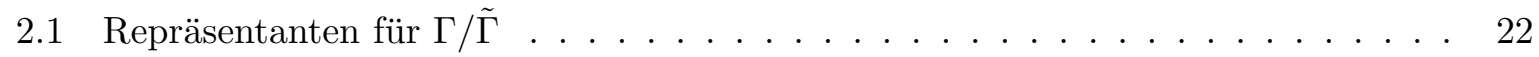

2.2 Repräsentanten für $\Gamma / \tilde{\Gamma}$ nach $[10] \ldots \ldots \ldots \ldots \ldots \ldots$

3.1 Liste der automorphen Eigenwerte . . . . . . . . . . . . . . . . . . 42

3.2 Liste der ersten fünfundfünzig $c(p)$ für $R \in\left\{R_{1}^{+}, R_{1}^{-}, R_{2}^{-}, R_{14}^{-}\right\} \ldots \ldots \ldots$

4.1 Nichttriviale Nachbarschaften . . . . . . . . . . . . . . . . . . . 55

5.1 Liste der Eigenwerte zu $\tilde{\Gamma} \ldots \ldots \ldots \ldots$. . . . . . . . . . . . 60

5.2 Metaplektische und automorphe Fourierentwicklung für $R=R_{3}^{-} \ldots \ldots \ldots$

5.3 Metaplektische und automorphe Fourierentwicklung für $R=R_{6}^{+} \ldots \ldots$. . . 66

5.4 Metaplektische und automorphe Fourierentwicklung für $R=R_{15}^{+}(1) \ldots \ldots$. . . 67

5.5 Metaplektische und automorphe Fourierentwicklung für $R=R_{15}^{+}(2) \ldots \ldots$. . . 68

$5.6 A^{3}(n)$ und $L_{f}(s, \chi)$ für $a=0, b=0$ und $R=R_{1}^{+} \ldots \ldots \ldots \ldots \ldots$

$5.7 A^{3}(n)$ und $L_{f}(s, \chi)$ für $a=0, b=0$ und $R=R_{2}^{+} \ldots \ldots \ldots \ldots$

$5.8 A^{3}(n)$ und $L_{f}(s, \chi)$ für $a=0, b=0$ und $R=R_{1}^{-} \ldots \ldots \ldots \ldots$ 


\title{
Liste häufig verwendeter Symbole
}

\author{
$(\cdot / \cdot)_{3}=(\cdot)_{3}$ das kubische Restsymbol in $\mathbb{Q}(\omega)$ \\ $\langle\cdot, \cdot\rangle \quad$ das Skalarprodukt $\langle w, z\rangle=(w \bar{z}+\bar{w} z) / 2=\operatorname{Re} w \bar{z}$ \\ $\bar{z} \quad$ das komplex Konjugierte von $z$ für $z \in \mathbb{C}$ \\ $|z|=\sqrt{z \bar{z}} \quad$ der Absolutbetrag von $z$ für $z \in \mathbb{C}$ \\ $|\gamma|=\operatorname{det}(\gamma)$ die Determinante von $\gamma$, falls $\gamma$ eine lineare Abbildung \\ $A=\sqrt{3} / 2$ die Fläche der Fundamentalzelle von $\operatorname{PSL}(2, \mathrm{Z}[\omega])$ in der $z$-Ebene \\ $\mathbb{C}, \mathbb{I N}, \mathbb{Q}, Z$, die komplexen, natürlichen, rationalen und ganzen Zahlen \\ $e(z)=e^{2 \pi i z} \quad$ die Exponentialfunktion \\ $\mathbb{H}^{3} \quad$ der hyperbolische obere Halbraum \\ $K_{\nu}(z) \quad$ die K-Bessel Funktion zum Index $\nu$ mit Argument $z$ \\ $N(\alpha)=\|\alpha\|$ die (algebraische) Norm von einer algebraisch rationalen Zahl $\alpha$ \\ $\omega=\frac{-1+\sqrt{-3}}{2}$ eine primitive dritte Einheitswurzel, eingebettet in $\mathbb{C}$ durch $\sqrt{-1}=i$ \\ $\omega_{6}=\omega+1 \quad$ eine primitive sechste Einheitswurzel
}




\section{Kapitel 1}

\section{Einleitung}

Die vorliegende Arbeit befaßt sich mit der Fourierentwicklung von automorphen Formen auf dem oberen Halbraum $\mathbb{H}^{3}$. Auf diesem Raum operiert PGL $(2, \mathbb{C})$ transitiv, und automorphe Formen sind Eigenfunktionen des nichteuklidischen Laplaceoperators, welche sich höchstens mit einem Charakter unter Untergruppen von PSL(2, C) transformieren. Sie haben eine Fourierentwicklung der Form

$$
f(z, r)=\sum_{n \in \Lambda}^{\prime} a_{n} r K_{i R}(2 \pi|n| r) e(\langle n, z\rangle)
$$

zu einem Gitter $\Lambda$. In [10] wird eine bijektive Korrespondenz zwischen den automorphen Formen zur Untergruppe PSL $(2, \mathrm{Z}[\omega])$ mit trivialem Charakter und denen zu einer metaplektischen Untergruppe $\tilde{\Gamma}$ von $\operatorname{PSL}(2, \mathrm{Z}[\omega])$ mit Kubotacharakter aufgezeigt.

Während es bereits die beiden Arbeiten [19] und [2] gibt, welche die automorphen Formen auf $\operatorname{PSL}(2, Z[\omega])$ numerisch zu ergründen suchen, bestand für $\tilde{\Gamma}$ noch die Notwendigkeit einer Untersuchung. Mit einer allgemeinen Kollokationsmethode, welche - auf den einfachen Fall von $\operatorname{PSL}(2, Z[\omega])$ angewandt - die Genauigkeit der Ergebnisse von Steil unterstreichen konnte, ist es möglich, auch automorphe Formen auf $\tilde{\Gamma}$ numerisch zu untersuchen. Die vorhergesagte Bijektion zwischen automorphen und metaplektischen Formen konnte im Untersuchungsbereich bestätigt werden.

Im Kapitel 2 werden Vorarbeiten für die Erstellung eines Algorithmus geleistet. Neben einigen Zusammenfassungen zählt dazu auch die Bestimmung eines günstigen Fundamentalbereiches der betrachteten metaplektischen Gruppe.

Mit diesen Vorbereitungen kann dann in Kapitel 3 die Kollokation im automorphen Fall 
durchgeführt werden. Das Kapitel 4 führt eine möglichst weitgehende Auflösung von Redundanzen in der Fourierdarstellung im metaplektischen Fall durch und analysiert die Kollokationsbereiche.

Analog zu Vermutungen in ähnlich gelagerten Fällen, wie [21] oder spezieller [6], taucht die Frage nach Zusammenhängen der zur automorphen Gruppe gehörenden $L$-Reihe und den Fourierkoeffizienten der korrespondierenden metaplektischen Form auf. Diese Frage wird in Kapitel 5 untersucht.

Der überwiegende Teil der Algorithmen wurde als GP-Scripte, in GP-Pari [8] (vergleiche http://pari.home.ml.org) umgesetzt, lediglich die zeit- und speicherplatzkritischen Berechnungen der Eigenwerte zu metaplektischen Formen sind in C++ realisiert. Zum Vergleich wurden einige zusätzliche Genauigkeitstests mit MuPAD (http://www.MuPAD.de) von B. Fuchssteiner et. al. ausgeführt.

Die Funktionsgraphen wurden mit dem Programm Gnuplot von Th. Williams und C. Kelley et. al. (1998), vergleiche http://www.gnuplot.info, erstellt.

Ich möchte an dieser Stelle Herrn Prof. S. J. Patterson sehr herzlich für die Vergabe dieses interessanten Themas und für die engagierte Betreuung danken.

\section{Inhalt der CD}

Auf der beigelegten CD befinden sich im Verzeichnis DOC weitere Tabellen und Abbildungen als Postscript-Dateien. In dem Verzeichnis CHAP3 sind die in Kapitel 3 beschriebenen Koeffizienten und Eigenwerte als Rohdaten zu finden, im Verzeichnis CHAP5 die entsprechenden Werte zu Kapitel 5. Die Daten zu den Plots und deren Scripte befinden sich im Verzeichnis GNUPLOT, um auch die Betrachtung anderer als der gewählten Ausschnitte der Funktionen zu ermöglichen. In allen Verzeichnissen gibt es eine Datei INDEX.TXT, welche die Struktur der entsprechenden Dateien näher beschreibt. 


\section{Kapitel 2}

\section{Gruppentheorie und automorphe Formen}

\subsection{Die Automorphe Gruppe}

Sei $\mathbb{H}^{3}=\mathbb{C} \times \mathbb{R}^{+}$der obere Halbraum, Punkte darin seien mit $w=(z, v) ; z=x+i y, v>0$ bezeichnet. Die Hyperbolische Metrik erhält man durch

$$
d s^{2}=\frac{d x^{2}+d y^{2}+d v^{2}}{v^{2}} \text { mit dem Volumenelement } d V=\frac{d x d y d v}{v^{3}} .
$$

Geodäten oder hyperbolische Geraden sind dann Halbkreise und Halbgeraden, die orthogonal auf $\mathbb{C} \times\{0\}$ sind; hyberbolische Ebenen sind dementsprechend Halbkugeln und Halbebenen, die orthogonal auf $\mathbb{C} \times\{0\}$ stehen. Der nichteuklidische Laplace-Operator wird zu

$$
\Delta=v^{2}\left(\Delta_{\text {euk }}+\frac{\partial^{2}}{\partial v^{2}}\right)-v \frac{\partial}{\partial v} \quad \text { mit } \quad \Delta_{\text {euk }}=\left(\frac{\partial^{2}}{\partial x^{2}}+\frac{\partial^{2}}{\partial y^{2}}\right) .
$$

Die Gruppe GL $(2, \mathbb{C})$ operiert auf $\mathbb{H}^{3}$ durch

$$
\left(\begin{array}{ll}
a & b \\
c & d
\end{array}\right)(z, v)=\left(\frac{(a z+b) \overline{(c z+d)}+a \bar{c} v^{2}}{|(c z+d)|^{2}+|c|^{2} v^{2}}, \frac{v\left|\left(\begin{array}{ll}
a & b \\
c & d
\end{array}\right)\right|}{|(c z+d)|^{2}+|c|^{2} v^{2}}\right) .
$$

Für $\left(\begin{array}{ll}a & b \\ 0 & d\end{array}\right) \in S L(2, \mathcal{O})$ gilt $|d|=1$, falls $\mathcal{O}$ ein Dedekindring ist, und $(2.2)$ vereinfacht sich zu

$$
\left(\begin{array}{ll}
a & b \\
0 & d
\end{array}\right)(z, v)=\left(\frac{a z+b}{d}, v\right)
$$

Allgemeiner gilt: Sei

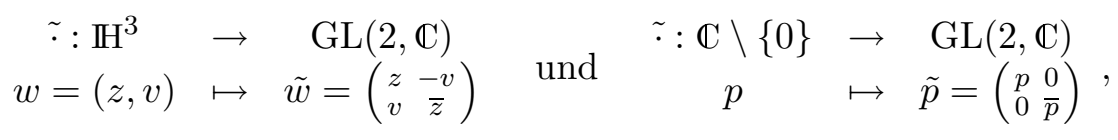


dann ist mit $\gamma=\left(\begin{array}{ll}a & b \\ c & d\end{array}\right)$ :

$$
\widetilde{\gamma w}=(\tilde{a} \tilde{w}+\tilde{b})(\tilde{c} \tilde{w}+\tilde{d})^{-1}
$$

Dieses Modell entspricht genau der Einbettung von $\mathbb{H}^{3}$ in die Quaternionen durch $(z, v) \mapsto$ $x+i y+j v$ mit der üblichen Schreibweise $i^{2}=j^{2}=k^{2}=-1$ und $i j=k, j k=i$ und $k i=j$. In Anlehnung an die klassische Theorie gebrochen rationaler Transformationen werden diese Abbildungen Automorphien und Gruppen dieser Elemente automorph genannt. Man prüft leicht nach, daß die komplexen gebrochenen rationalen Transformationen Isometrien und invariant unter $-I=\left(\begin{array}{cc}-1 & 0 \\ 0 & -1\end{array}\right)$ sind. Tatsächlich wird mit diesen Operationen die ganze Gruppe $\operatorname{Iso}^{+}\left(\mathbb{H}^{3}\right)$ der orientierungserhaltenden Isometrien erreicht [13], und so werden in dieser Arbeit entsprechende Matrizen aus $\operatorname{Iso}^{+}\left(\mathbb{H}^{3}\right)=\operatorname{PSL}(2, \mathbb{C})=\mathrm{SL}(2, \mathbb{C}) /\{ \pm I\}$ mit ihren Restklassen identifiziert. Gleichheit von Matrizen soll also Äquivalenz modulo $\pm I$ bedeuten. Im folgenden werden einige spezielle Untergruppen von $\operatorname{PGL}(2, \mathbb{C})$ betrachtet, nämlich $\operatorname{PGL}(2, Z[\omega])$ und $\operatorname{PSL}(2, Z[\omega])$, wobei $\mathrm{Z}[\omega]$ der Ring der zu $\mathbb{Q}(\sqrt{-3})$ gehörenden ganzen Zahlen ist, wobei $\omega=\frac{-1+\sqrt{-3}}{2}$ ist. Einheiten in $\mathrm{Z}[\omega]$ sind $\left\{(-\omega)^{i}\right\}_{0 \leq i \leq 5}$. Die diese Gruppe erzeugende Einheit $(-\omega)^{5}=e^{2 \pi i / 6}=\omega+1$ soll im folgenden $\omega_{6}$ genannt werden.

Die Gruppe PSL $(2, Z[\omega])$ ist eine Bianchi-Gruppe [1]. Sie ist eine diskrete Untergruppe von $\operatorname{PSL}(2, \mathbb{C})$ und operiert diskontinuierlich auf $\mathbb{H}^{3}$. Man kann daher, ohne hier auf die Probleme des Randes näher einzugehen, als Fundamentalbereich $\mathcal{F} \cong \operatorname{PSL}(2, \mathrm{Z}[\omega]) \backslash \mathbb{H}^{3}$ ein hyperbolisches Polyeder wählen, welches ein endliches hyperbolisches Volumen (unabhängig von der Wahl des Polyeders) hat, aber nicht kompakt ist, da Eckpunkte des Polyeders außerhalb von $\mathbb{H}^{3}$ liegen. Diese sogenannten "Spitzen” sind entweder der Punkt bei $v \rightarrow \infty$, der als Punkt $\infty$ genannt werden soll, oder ein Punkt $w \in \mathbb{Q}[\omega] \times\{0\}$ in der $z$-Ebene, der als endliche Spitze abkürzend mit seiner $z$-Komponente identifiziert werden soll. Tatsächlich sind alle diese Spitzen PSL $(2, Z[\omega])$ äquivalent, so daß man einen Fundamentalbereich wählen kann, für den genau ein Eckpunkt eine Spitze ist. $\operatorname{PSL}(2, Z[\omega])$ wird von den Transformationen

$$
\begin{aligned}
& T=\left(\begin{array}{cc}
1 & 1 \\
0 & 1
\end{array}\right):(z, v) \mapsto(z+1, v), \\
& S=\left(\begin{array}{cc}
0 & 1 \\
-1 & 0
\end{array}\right):(z, v) \mapsto\left(\frac{-\bar{z}}{|z|^{2}+v^{2}}, \frac{v}{|z|^{2}+v^{2}}\right), \\
& R=\left(\begin{array}{cc}
\omega^{2} & 0 \\
0 & \omega
\end{array}\right):(z, v) \mapsto(\omega z, v)
\end{aligned}
$$


erzeugt. Da $\omega=e^{2 \pi i / 3}$ eine Einheit in $\mathrm{Z}[\omega]$ ist, entspricht $R$ einer $2 \pi / 3$-Drehung um die Achse $z=0$ und damit gilt $R^{3}=1$. Die Drehung der $z$-Ebene um $2 \pi / 6$ ist dagegen nicht in $\operatorname{PSL}(2, \mathrm{Z}[\omega])$. Weiter ist $S$ eine Spiegelung um $(0,1)$ entlang $|w|=1$ mit $S^{2}=1$ und $T$ eine Translation der $z$-Ebene um 1. Die Isometrien $T, S$ und $R$ erzeugen $\operatorname{PSL}(2, Z[\omega])$. Dies läßt sich leicht nachprüfen, da $\mathrm{Z}[\omega]$ euklidisch ist. Figur 2.1 zeigt den Fundamentalbereich $\mathcal{F}_{3}$ zur Spitze $\infty$ und dessen Projektion in die z-Ebene, wobei das Innere von $\mathcal{F}_{3}$ durch

$$
\stackrel{\circ}{\mathcal{F}}_{3}=\left\{(x+i y, v) \in \mathbb{H}^{3}|| x^{2}+y^{2}+v^{2} \mid>1,0<x<1 / 2,-x \frac{\sqrt{3}}{3}<y<(1-x) \frac{\sqrt{3}}{3}\right\}
$$

beschrieben wird.

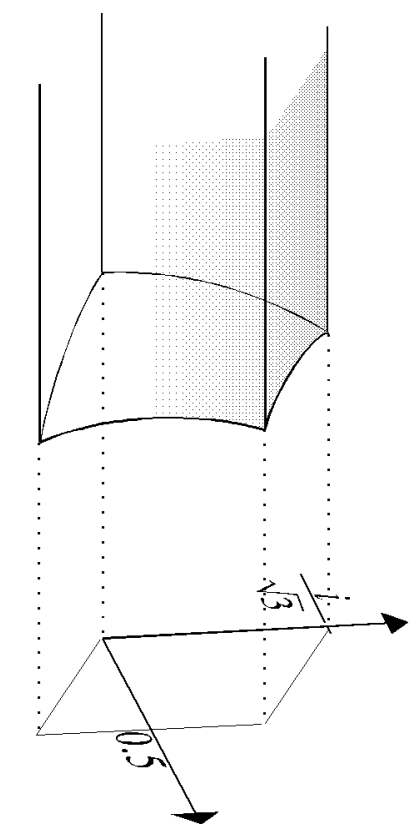

Abbildung 2.1: Der Fundamentalbereich $\mathcal{F}_{3}$ von $\Gamma=\operatorname{PSL}(2, \mathrm{Z}[\omega])$

Daß dies ein Fundamentalbereich ist, erkennt man beispielsweise daran, daß diejenigen Abbildungen, welche Randflächen von $\mathcal{F}_{3}$ mit Randflächen identifizieren, die Abbildungen $R S, R$, $R^{2}, T R^{2}$ und $R T^{-1}$ sind, welche offenbar $R, S$ und $T$ und damit PSL(2, Z $\left.[\omega]\right)$ erzeugen. Wie man sieht, ist der gewählte Fundamentalbereich, und damit auch seine Kopien, eine hyperbolische Pyramide mit einer viereckigen Grundfläche und dementsprechend vier dreieckigen Seitenflächen, von denen eine Ecke immer in einer Spitze liegt. Der weiteren Veranschaulichung möge Abbildung 2.2 dienen, welche (a) die benachbarten Bereiche $\mathcal{F}_{3}, R \mathcal{F}_{3}$, und $R^{2} \mathcal{F}_{3}$, sowie (b) $R S \mathcal{F}_{3}$ 

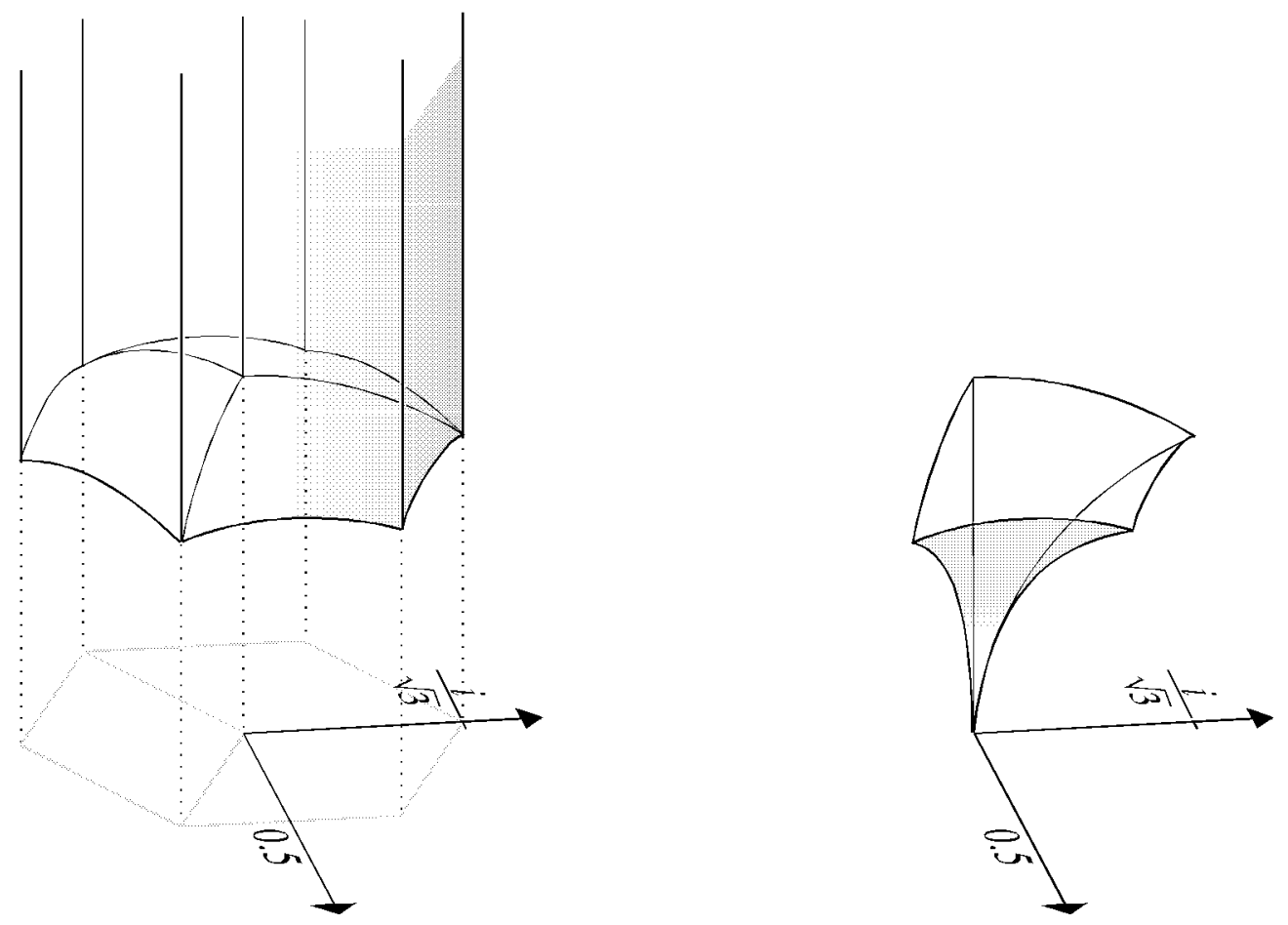

Abbildung 2.2: (a) Die Bereiche $\mathcal{F}_{3}, R \mathcal{F}_{3}, R^{2} \mathcal{F}_{3}$, und 2.2(b) der Bereich $R S \mathcal{F}_{3}$

zeigt. Letzterer ist eine Kopie von $\mathcal{F}_{3}$ zur Spitze 0, welche entlang einer viereckigen Seite zu $\mathcal{F}_{3}$ benachbart ist. Die ersten drei Bereiche in Figur 2.2 (a) sind dadurch ausgezeichnet, daß deren drei „endliche” Flächen, also diejenigen Flächen, welche nicht die Spitze $\infty$ berühren, in einer hyperbolischen Ebene liegen. Dies entspricht der Gruppeneigenschaft von $(R)=\left\{1, R, R^{2}\right\}$, ein Normalteiler von $\operatorname{PSL}(2, \mathrm{Z}[\omega])$ zu sein. Dadurch lassen sich Dreiergruppen von Bereichen zu Pyramiden mit einer sechseckigen Grundseite zusammenfassen, welche die Überdeckung

$$
\mathbb{H}^{3}=\bigcup_{\gamma \in \Gamma /(R)}^{\circ}\left(\gamma \underset{i \in\{0,1,2\}}{\bigcup^{\prime}} R^{i} \mathcal{F}_{3}\right)
$$

erzeugen, und zu denen die Überdeckung von $\mathbb{H}^{3}$ durch $\bigcup_{\gamma \in \Gamma} \gamma \mathcal{F}_{3}$ eine Verfeinerung ist. In Abbildung 2.3 auf Seite 23 sind neun solche Dreiergruppen zu sehen. Die Grundstruktur dreier viereckiger Grundflächen, welche eine sechseckige Grundfläche ergeben, ist in Figur 2.2 (a) bereits an der Projektion der Grundflächen in die $z$-Ebene gut zu erkennen. 


\subsection{Automorphe Formen auf $\operatorname{PSL}(2, \mathrm{z}[\omega])$}

Sei $\Gamma=P S L(2, \mathrm{Z}[\omega])$ und $L^{2}\left(\Gamma \backslash \mathbb{H}^{3}\right)$ der Raum von $\Gamma$-invarianten quadratintegrierbaren Funktionen auf $\mathbb{H}^{3}$. Hierbei operiert $\Gamma$ auf $f$ punktweise, das heißt $\gamma f((z, v))=f(\gamma(z, v))$. Quadratintegrierbar ist hier bezüglich des Petersson Skalarprodukts

$$
(f, g)=\int_{\mathcal{F}_{3}} f \bar{g} d V
$$

gemeint. Dies ist unabhängig von der Wahl von $\mathcal{F}_{3}$, da $d V$ invariant unter $\mathrm{SL}(2, \mathbb{C})$ ist. Der Laplace-Operator $-\Delta$ aus (2.1) ist auf diesem Raum positiv und selbstadjungiert. Das Spektrum besteht aus einem kontinuierlichen und einem diskreten Anteil, wobei der erstere das Intervall $[1, \infty)$ mit Vielfachheit 1 ist. Der diskrete Anteil ist von der Form

$$
0=\lambda_{0}<1<\lambda_{1} \leq \lambda_{2} \leq \ldots ; \quad \lambda_{i} \rightarrow \infty(i \rightarrow \infty)
$$

$\lambda_{0}$ korrespondiert dabei mit den konstanten Funktionen, für $1<\lambda_{1}$ vergleiche [20]. Die dazu gehörenden nichtkonstanten Eigenformen heißen automorphe Formen, genauer: Eine automorphe Form ist eine Funktion aus $L^{2}\left(\Gamma \backslash \mathbb{H}^{3}\right)$, welche in den Spitzen höchstens polynomial wächst und einer Gleichung

$$
-\Delta f=\lambda f=\left(1+R^{2}\right) f
$$

mit $R>0$ genügt. Der Parameter $R$ bestimmt den Eigenwert $\lambda$ eindeutig und wird im folgenden als beschreibende Größe für die Angabe des Eigenwertes gebraucht. Die automorphen Formen, die in den Spitzen verschwinden, heißen Spitzenformen. Die Menge der Spitzenformen wird mit $\mathcal{S}(\Gamma)$ bezeichnet.

Der Raum $\mathcal{S}(\Gamma)$ zerlegt sich in eine orthogonale Summe von vier Symmetrien: Der nichteuklidische Laplace-Operator kommutiert mit Drehungen und Spiegelungen in der $z$-Koordinate und es gibt zwei unabhängige Bewegungen, welche nicht in $\operatorname{PSL}(2, Z[\omega])$ enthalten sind und das $\mathrm{zu} \mathcal{O}=\mathrm{Z}[\omega]$ duale Gitter fixieren, etwa die Drehung $U_{0}:(z, v) \mapsto\left(\omega_{6} z, v\right)$ und die Spiegelung $U_{1}=\bar{\varepsilon}:(z, v) \mapsto(-\bar{z}, v)$. Mit $\omega_{6}=\omega-1$ und $\bar{\omega}=1 / \omega$ und

$$
U_{0}^{-1}\left(\begin{array}{ll}
a & b \\
c & d
\end{array}\right) U_{0}=\left(\begin{array}{cc}
a & b / \omega_{6} \\
c \omega_{6} & d
\end{array}\right) \quad \text { und } \quad U_{1}^{-1}\left(\begin{array}{ll}
a & b \\
c & d
\end{array}\right) U_{1}=\left(\begin{array}{cc}
\bar{a} & -\bar{b} \\
-\bar{c} & \bar{d}
\end{array}\right)
$$


sind dies äußere Automorphismen von $\operatorname{PSL}(2, \mathrm{Z}[\omega])$, deren Quadrat in $\operatorname{PSL}(2, \mathrm{Z}[\omega])$ liegt. Für $f \in L^{2}\left(\Gamma \backslash \mathbb{H}^{3}\right)$ ist daher $U_{\nu}^{2} f=f$ und

$$
U_{\nu}\left(f \pm U_{\nu} f\right)= \pm\left(f \pm U_{\nu} f\right)
$$

Da $\Delta$ und $U_{\nu}$ kommutieren, ist mit $f$ auch $\left(f \pm U_{\nu} f\right)$ Eigenfunktion zu $\Delta$. Es können nicht sowohl $\left(f+U_{\nu} f\right)$ als auch $\left(f-U_{\nu} f\right)$ verschwinden, daher kann für eine Eigenfunktion $g$ ohne Einschränkung angenommen werden, daß $U_{\nu} g=g$ oder $U_{\nu} g=-g$ gilt. Bezeichne $D$ den Raum, der in allen Symmetrien $U_{\nu}$ geraden Funktionen, $C$ die bezüglich $U_{0}$ ungeraden, $G$ die bezüglich $U_{1}$ ungeraden und $H$ die in beiden Symmetrien ungeraden, so ergibt sich

$$
\mathcal{S}(\Gamma)=D \oplus C \oplus G \oplus H
$$

Der Einfachheit halber sollen Funktionen aus $D \oplus G$ im folgenden $\omega_{6}$-gerade oder einfach gerade (da $\omega_{6}=-\omega^{2}$ ), solche aus $C \oplus H$ entsprechend $\omega_{6}$-ungerade oder ungerade heißen.

Eine Fourierentwicklung erhält man am einfachsten durch Variablenseparation. Es sei:

$$
\Delta f(w)=-\lambda f(w) \quad \text { und } \quad f(w)=g(z) h(v) \text { mit } w=(z, v) .
$$

Für $h$ ergibt sich damit die Differentialgleichung

$$
-\lambda h(v)=v^{2} h^{\prime \prime}(v)+v^{2} h(v) \frac{\Delta_{\text {euk }} g(z)}{g(z)}-v h^{\prime}(v)
$$

und damit ist $g(z)$ Eigenfunktion zum euklidischen Laplace-Operator: $\lambda^{\prime} g(z)=\Delta_{\text {euk }} g(z)$ und zusammen mit der Periodizität von $f(z, v)$ bezüglich $f(z, v) \mapsto f(z+n, v), n \in \mathcal{O}$ folgt

$$
g_{n}(z)=e(\langle n, z\rangle)
$$

Die Gleichungen (2.8) und (2.7) ergeben

$$
v^{2} h_{n}^{\prime \prime}(v)-v h_{n}^{\prime}(v)=\left((2 \pi|n| v)^{2}-\lambda\right) h_{n}(v) .
$$

Dies ist aber genau die Differentialgleichung der $K$-Besselfunktion mit $h_{n}(v)=v K_{s-1}(2 \pi|n| v)$ und $\lambda=s(2-s)$. Damit ergibt sich die Fourierentwicklung

$$
\begin{aligned}
f(z, v) & =\sum_{n \in \Lambda}^{\prime} a_{n} v K_{i R}(2 \pi|n| v) e(\langle n, z\rangle) \\
& =\sum_{n \in \mathcal{O}}^{\prime} a^{\prime}(n) v K_{i R}\left(\frac{2 \pi}{A}|n| v\right) e\left(\frac{\langle i n, z\rangle}{A}\right),
\end{aligned}
$$


wobei $\Lambda$ das zu $\mathcal{O}$ duale Gitter, $\lambda=\left(1+R^{2}\right)=s(2-s), K_{\nu}(z)$ die modifizierte Besselfunktion, $e(z)=e^{2 \pi i z}$ und $\langle\eta, z\rangle=(\eta \bar{z}+\bar{\eta} z) / 2$ sowie $A=\sqrt{3} / 2$ ist. Da die Indizierung der Koeffizienten in (2.11) etwas einfacher als in (2.10) ist, soll hier [19] folgend diese Variante der üblicheren ersten Schreibweise vorgezogen werden. Für die Fourierkoeffizienten ergibt sich bei Transformationen direkt

$$
(z, v) \mapsto(\varepsilon z, v) \Leftrightarrow a^{\prime}(n) \mapsto a^{\prime}(\varepsilon n) \quad \text { und } \quad(z, v) \mapsto(-\bar{z}, v) \Leftrightarrow a^{\prime}(n) \mapsto a^{\prime}(\bar{n})
$$

wobei $\varepsilon$ eine Einheit ist. Dies erlaubt offenbar, die Fourierentwicklung zu vereinfachen:

$$
f(z, v)=\sum_{n \in \mathcal{O} / \mathcal{O}^{\times}}^{\prime} c(n) v K_{i R}\left(\frac{2 \pi}{A}|n| v\right) \operatorname{cs}(n, z)
$$

wobei für $\omega_{6}$-gerade Spitzenformen

$$
\operatorname{cs}(n, z)=\cos \left(\frac{2 \pi}{A}\langle i n, z\rangle\right)+\cos \left(\frac{2 \pi}{A}\langle i \omega n, z\rangle\right)+\cos \left(\frac{2 \pi}{A}\langle i(\omega+1) n, z\rangle\right)
$$

und für $\omega_{6}$-ungerade entsprechend

$$
\operatorname{cs}(n, z)=\sin \left(\frac{2 \pi}{A}\langle i n, z\rangle\right)+\sin \left(\frac{2 \pi}{A}\langle i \omega n, z\rangle\right)-\sin \left(\frac{2 \pi}{A}\langle i(\omega+1) n, z\rangle\right)
$$

gilt. Dabei entsprechen die $c(n)$ den um den Faktor 2 bzw. $2 i$ reskalierten $a^{\prime}(n)$.

Auf diesem Raum gibt es, ganz analog zum klassischen Fall automorpher Funktionen, eine Hecketheorie: Es gibt für $m \in \mathrm{Z}[\omega]$ einen kommutativen Ring von Operatoren $T_{m}$, definiert durch

$$
T_{m} f=\frac{1}{|m|} \sum_{\substack{a d=m \\
b \bmod (d)}}^{\times} f \circ\left(\begin{array}{ll}
a & b \\
0 & d
\end{array}\right),
$$

welche mit dem nichteuklidischen Laplace-Operator kommutieren. In dem Raum der Eigenfunktionen von $\Delta$ zu einem Eigenwert läßt sich daher eine Basis von Eigenfunktionen finden, welche für alle diese Operatoren gleichzeitig Eigenfunktionen sind, das heißt, man kann Spitzenformen mit $T_{m} f=t_{m} f$ wählen. Mit den Definitionsgleichungen der Heckeoperatoren gilt direkt $t_{m} c(n)=\sum_{d \mid(\bar{m}, n)}^{\times} c\left(\frac{\bar{m} n}{d^{2}}\right)$ und mit $t_{m} c(1)=c(\bar{m})$ gelten die sogenannten Heckerelationen

$$
c(m) c(n)=c(1) \sum_{d \mid(m, n)}^{\times} c\left(\frac{m n}{d^{2}}\right) .
$$


Dementsprechend kann man eine nicht verschwindende Spitzenform durch $c(1)=1$ normalisieren, und - ebenfalls genau wie im klassischen Fall - durch die Formel

$$
c(m p)=c(m) c(p)-c(m / p) \quad \text { mit } \quad c(m / p)=0 \text { falls } p \nmid m
$$

alle Fourierkoeffizienten aus den Fourierkoeffizienten an den Primstellen berechnen.

Außerdem gilt für Einheiten $\mu$

$$
T_{\mu} f(z, v)=f(\mu z, v)
$$

und damit $T_{\omega-1}=U_{0}$, wobei $U_{\nu}$ die in (2.6) definierten Symmetrien sind. Um festzustellen, ob Eigenfunktionen zu $\Delta$ und den $T_{m}$ gleichzeitig ohne Beschränkung der Allgemeinheit die Symmetrien $U_{\nu}$ erfüllen können, ist zu prüfen, ob die $U_{\nu}$ und die $T_{m}$ kommutieren. Es folgt aus $T_{\omega-1}=U_{0}$ sofort $T_{m} U_{0}=U_{0} T_{m}$, während man (vgl. [19]) an der Definition (2.14) direkt $T_{m} U_{1} f=U_{1} T_{\bar{m}} f$ ablesen kann, so daß für die Entwicklung der Eigenfunktionen nur $U_{0} f= \pm f$ gefordert werden kann, wie in den Gleichungen (2.13) und $\left(2.13^{\prime}\right)$ bereits geschehen. Steil zeigt weiter, daß

$$
\left.\forall \lambda \in \mathbb{R}^{+}, f \in G \text { (bzw. } H\right): \lambda f=f \Rightarrow \exists g \in D \text { (bzw. } C \text { ) }: \lambda g=g
$$

folgt, also daß für Eigenfunktionen in $G$ bzw. $H$ die Eigenwerte doppelt mit einer weiteren Eigenfunktion in $D$ bzw. $C$ auftauchen. Numerisch finden sich neben diesen doppelten keine weiteren mehrfachen Eigenwerte. 


\subsection{Die Metaplektische Gruppe}

An dieser Stelle sollen nur die elementaren Aspekte der Theorie der metaplektischen Überlagerungen wiederholt werden, welche im Rahmen der Berechungen benötigt werden. Dieses Kapitel folgt in Teilen [10] und [15]. Sei also wie gehabt $\omega=\frac{-1+\sqrt{-3}}{2}$ eine dritte, $\omega_{6}=\frac{1+\sqrt{-3}}{2}$ eine sechste Einheitswurzel, also $\mathbb{Q}(\omega)=\mathbb{Q}\left(\omega_{6}\right)$ und $\left(1, \omega_{6}\right)$ ist die „kanonische” Ganzheitsbasis zum quadratischen Zahlkörper mit Diskriminante -3 . Außerdem seien:

$$
\begin{gathered}
\Gamma=\operatorname{PSL}(2, \mathrm{Z}[\omega]), \\
\Gamma(3)=\{\gamma \in \Gamma \mid \gamma \equiv I \quad(\bmod 3)\}
\end{gathered}
$$

und

$$
\tilde{\Gamma}=\{\gamma \in \Gamma \mid \exists g \in \operatorname{PSL}(2, \mathrm{Z}): \gamma \equiv g \quad(\bmod 3)\}
$$

und sei $(a / b)_{3}$ das kubische Restsymbol auf $\mathbb{Q}\left(\omega_{3}\right)$. Dann ist $\Gamma$ automorph wie in Kapitel 2.1, und $\tilde{\Gamma}$ metaplektisch im Sinne von [10]. Da im folgenden nur diese Gruppen intensiv betrachtet werden, soll der Einfachheit halber $\Gamma$ die automorphe Gruppe, und $\tilde{\Gamma}$ die metaplektische Gruppe genannt werden.

Es ist $[\Gamma: \tilde{\Gamma}]=27$. Dies erkennt man beispielsweise, indem man die Beziehung zwischen $\Gamma, \tilde{\Gamma}$ und $\Gamma(3)$ betrachtet: Es ist $\Gamma / \Gamma(3) \cong S L_{2}(\mathrm{Z} / 9 \mathrm{Z})$ und damit $[\Gamma: \Gamma(3)]=648$, außerdem ist $\tilde{\Gamma} / \Gamma(3) \cong S L_{2}(\mathrm{Z} / 3 \mathrm{Z})$, also $[\tilde{\Gamma}: \Gamma(3)]=24$. Einen Fundamentalbereich $\widetilde{\mathcal{F}}_{3}$ zu $\tilde{\Gamma}$ kann man erhalten, indem man ein beliebiges Vertretersystem $G=\left(\gamma_{i}\right)_{i}$ betrachtet und $\widetilde{\mathcal{F}}_{3}=\bigcup_{i} \gamma_{i} \mathcal{F}_{3}$ wählt. Die übliche Wahl von $\mathcal{F}_{3}$ wird dadurch bestimmt, daß man einen zusammenhängenden, nur zur Spitze $\infty$ gehörenden Bereich sucht, dessen Punkte minimale $z$-Komponente haben sollen, das heißt zu $(z, v) \in \gamma \mathcal{F}_{3}$ mit $\gamma: \infty \mapsto \infty$ gibt es kein $\gamma^{\prime} \in \Gamma$ mit $\left(z^{\prime}, v^{\prime}\right)=\gamma^{\prime}(z, v)$, $\gamma^{\prime}: \infty \mapsto \infty$ und $\left|z^{\prime}\right|<|z|$. Entsprechend soll die in dieser Arbeit getroffene Wahl der $\gamma_{i}$ einigen Prinzipien genügen:

- Es soll ein $i$ mit $\gamma_{i}=\mathrm{id}=\left(\begin{array}{ll}1 & 0 \\ 0 & 1\end{array}\right)$ geben. Dies entspricht der klassischen Wahl.

- Die Symmetrie $\varepsilon:(z, v) \mapsto(-z, v)$ soll sich in $\widetilde{\mathcal{F}}_{3}$ widerspiegeln. Da für alle Automorphismen $\phi$ mit $\mathcal{F}$ auch $\phi \mathcal{F}$ Fundamentalbereich ist, kann man für den äußeren Automorphismus $\varepsilon$ verlangen, daß $\varepsilon \widetilde{\mathcal{F}}_{3}=\widetilde{\mathcal{F}}_{3}$ gilt. 
- Die Invarianz bezüglich der Drehung $R$ soll sich in $\widetilde{\mathcal{F}}_{3}$ widerspiegeln. Um den Bereich $\widetilde{\mathcal{F}}_{3}$ zusammenhängend zu wählen, soll mit $\gamma_{i}$ auch $\gamma_{i} R$ im Vertretersystem $G$ enthalten sein.

- Unter diesen Voraussetzungen soll $\int_{\partial \widetilde{\mathcal{F}}_{\ni}} d s^{2}$ minimiert werden. Da $\int_{\partial \gamma \mathcal{F}_{3}} d s^{2}$ unabhängig von $\gamma$ ist, kann man induktiv aus diesen Punkten ein Vertretersystem bilden, welches so allerdings noch nicht eindeutig bestimmt ist. Um zu dem in Tabelle 2.1 dargestellten System $G$ zu kommen, muß zuerst mit id auch $R$ und $R^{2}$ in $G$ liegen. Dann kann man als endliche Spitzen entweder $\omega$ und $-\omega$ oder $\omega_{6}$ und $-\omega_{6}$ wählen. Schließlich gibt es eine Freiheit bei der Wahl der Vertreter zu den endlichen Spitzen (erkennbar etwa an den Abbildungen 2.5(b) und (c)), so daß es vier Vertretersyteme zu diesen Prinzipien gibt.

Sicher sind diese Maximen nicht alle kanonisch, allerdings erscheint keine ausgesprochen künstlich und zumindest zum Teil sind sie für die Numerik vorteilhaft, etwa die Symmetrie bezüglich $\varepsilon$ : Eine metaplektische Äquivalenz zweier Punkte $w=\gamma w^{\prime}$ mit $\gamma=\left(\begin{array}{ll}a & b \\ c & d\end{array}\right) \in \tilde{\Gamma}$ überträgt sich auch auf die durch $\varepsilon$ gedrehten Punkte, denn $\varepsilon w=\varepsilon \gamma \varepsilon^{-1}\left(\varepsilon w^{\prime}\right)$ und $\varepsilon \gamma \varepsilon^{-1}=$ $\varepsilon \gamma \varepsilon=\left(\begin{array}{cc}-a & b \\ c & -d\end{array}\right)=\left(\begin{array}{cc}a & -b \\ -c & d\end{array}\right)$. Ein Vertretersystem $\left(\gamma_{i}\right)_{i}$, welches symmetrisch unter $\varepsilon$ ist, läßt sich also am bequemsten dadurch wählen, daß $\forall i \exists j: \gamma_{j}=\varepsilon \gamma_{i} \varepsilon$ gilt. Das gewählte Vertretersystem für die 27 Restklassen zeigt Tabelle 2.1. Um die Tabelle etwas zu veranschaulichen, sind

\begin{tabular}{|c|c|c|c|c|c|}
\hline$j$ & $\gamma_{j}$ & $j$ & $\gamma_{j}$ & $j$ & $\gamma_{j}$ \\
\hline 1 & $\left(\begin{array}{ll}1 & 0 \\
0 & 1\end{array}\right)=I d$ & 10 & $\left(\begin{array}{cc}-1-\omega & -1 \\
1 & 0\end{array}\right)=L S$ & 19 & $\left(\begin{array}{cc}1+\omega & -1 \\
1 & 0\end{array}\right)=L^{-1} S$ \\
\hline 2 & $\left(\begin{array}{cc}-\omega & 0 \\
0 & 1+\omega\end{array}\right)=R^{2}$ & 11 & $\left(\begin{array}{cc}-1 & -1-\omega \\
-\omega & 0\end{array}\right)=L S R^{2}$ & 20 & $\left(\begin{array}{cc}1 & -1-\omega \\
-\omega & 0\end{array}\right)=L^{-1} S R^{2}$ \\
\hline 3 & $\left(\begin{array}{cc}-1-\omega & 0 \\
0 & \omega\end{array}\right)=R$ & 12 & $\left(\begin{array}{cc}\omega & -\omega \\
-1-\omega & 0\end{array}\right)=L S R$ & 21 & $\left(\begin{array}{cc}-\omega & -\omega \\
-1-\omega & 0\end{array}\right)=L^{-1} S R$ \\
\hline 4 & $\left(\begin{array}{cc}1 & 1+\omega \\
0 & 1\end{array}\right)=L^{-1}$ & 13 & $\left(\begin{array}{cc}-1-\omega & -2-\omega \\
1 & 1\end{array}\right)=L S T$ & 22 & $\left(\begin{array}{cc}1+\omega & -2-\omega \\
1 & -1\end{array}\right)=L^{-1} S T^{-1}$ \\
\hline 5 & $\left(\begin{array}{cc}-\omega & \omega \\
0 & 1+\omega\end{array}\right)=L^{-1} R^{2}$ & 14 & $\left(\begin{array}{cc}-1 & -1-2 \omega \\
-\omega & 1+\omega\end{array}\right)=L S T R^{2}$ & 23 & $\left(\begin{array}{cc}1 & -1-2 \omega \\
-\omega & -1-\omega\end{array}\right)=L^{-1} S T^{-1} R^{2}$ \\
\hline 6 & $\left(\begin{array}{cc}-1-\omega & -1 \\
0 & \omega\end{array}\right)=L^{-1} R$ & 15 & $\left(\begin{array}{cc}\omega & 1-\omega \\
-1-\omega & \omega\end{array}\right)=L S T R$ & 24 & $\left(\begin{array}{cc}-\omega & 1-\omega \\
-1-\omega & -\omega\end{array}\right)=L^{-1} S T^{-1} R$ \\
\hline 7 & $\left(\begin{array}{cc}1 & -1-\omega \\
0 & 1\end{array}\right)=L$ & 16 & $\left(\begin{array}{cc}-1-\omega & -2 \\
1 & -\omega\end{array}\right)=L S T L$ & 25 & $\left(\begin{array}{cc}1+\omega & -2 \\
1 & \omega\end{array}\right)=L^{-1} S T^{-1} L^{-1}$ \\
\hline 8 & $\left(\begin{array}{cc}-\omega & -\omega \\
0 & 1+\omega\end{array}\right)=L R^{2}$ & 17 & $\left(\begin{array}{cc}-1 & -2-2 \omega \\
-\omega & 1\end{array}\right)=L S T L R^{2}$ & 26 & $\left(\begin{array}{cc}1 & -2-2 \omega \\
-\omega & -1\end{array}\right)=L^{-1} S T^{-1} L^{-1} R^{2}$ \\
\hline 9 & $\left(\begin{array}{cc}-1-\omega & 1 \\
0 & \omega\end{array}\right)=L R$ & 18 & $\left(\begin{array}{cc}\omega & -2 \omega \\
-1-\omega & 1+\omega\end{array}\right)=L S T L R$ & 27 & $\left(\begin{array}{cc}-\omega & -2 \omega \\
-1-\omega & -1-\omega\end{array}\right)=L^{-1} S T^{-1} L^{-1} R$ \\
\hline
\end{tabular}

Tabelle 2.1: Repräsentanten für $\Gamma / \tilde{\Gamma}$ 
einige Elemente aus $\Gamma$ ausgezeichnet worden, und zwar $S, R$ und $T$ wie oben, und zusätzlich $L=R^{2} T R$, also

$$
S=\left(\begin{array}{cc}
0 & -1 \\
1 & 0
\end{array}\right) \quad R=\left(\begin{array}{cc}
\omega^{2} & 0 \\
0 & \omega
\end{array}\right) \quad T=\left(\begin{array}{ll}
1 & 1 \\
0 & 1
\end{array}\right) \quad L=\left(\begin{array}{cc}
1 & -\omega \\
0 & 1
\end{array}\right) .
$$

Der dazu gehörende Fundamentalbereich $\widetilde{\mathcal{F}}_{3}$ ist in Abbildung 2.3 dargestellt.

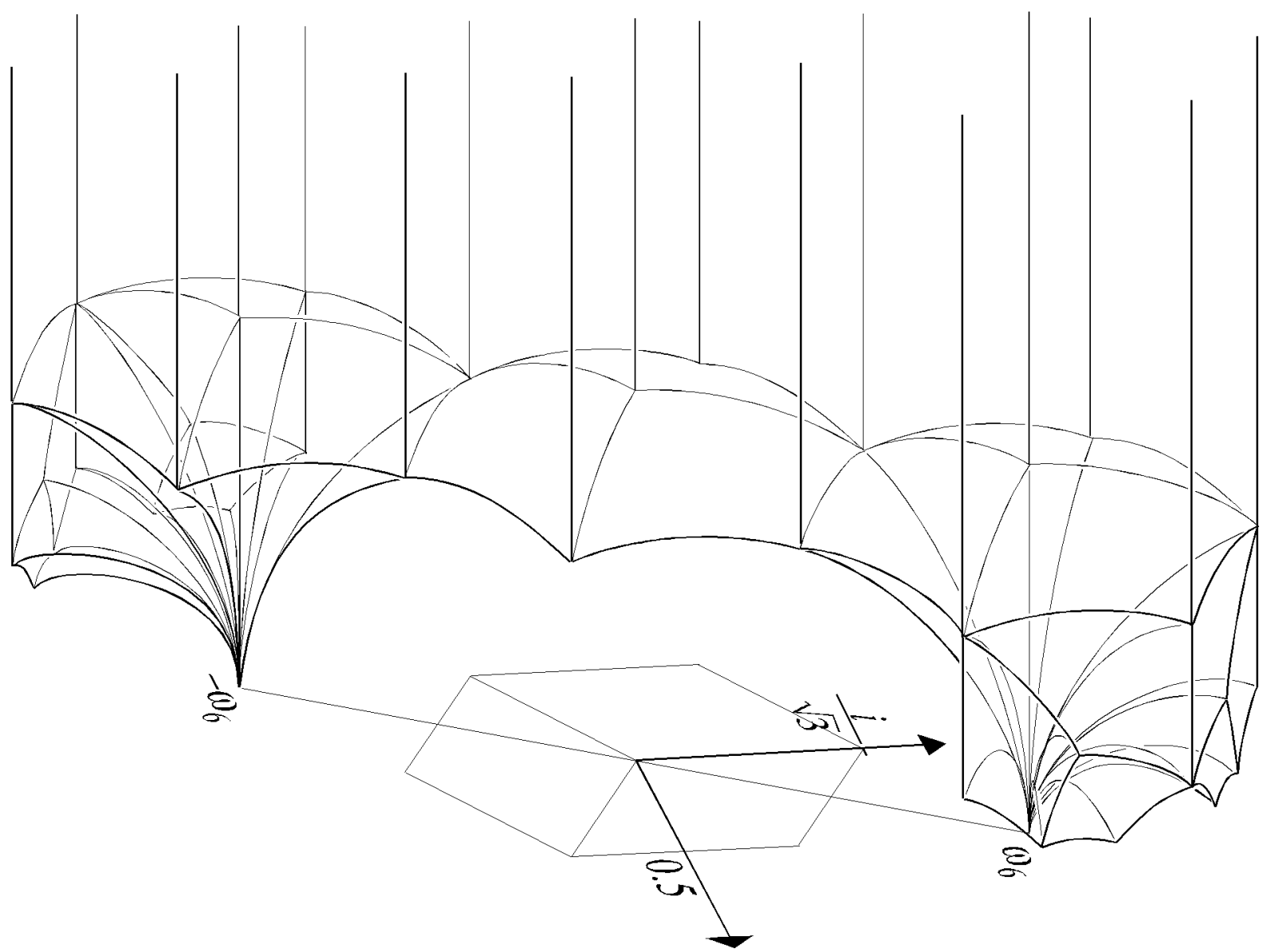

Abbildung 2.3: Der Fundamentalbereich $\widetilde{\mathcal{F}}_{3}=\bigcup_{i} \gamma_{i} \mathcal{F}_{3}$

Die Numerierung wurde so gewählt, daß gilt:

- Die Bereiche $\left(\gamma_{i} \mathcal{F}_{3}\right)_{\{1 \leq i \leq 9\}}$ berühren die Spitze $\infty$.

- Die Bereiche $\left(\gamma_{i} \mathcal{F}_{3}\right)_{\{10 \leq i \leq 18\}}$ berühren die Spitze $-\omega_{6}$.

- Die Bereiche $\left(\gamma_{i} \mathcal{F}_{3}\right)_{\{19 \leq i \leq 27\}}$ berühren die Spitze $\omega_{6}$. 
- Es gilt $\gamma_{i}=\varepsilon \gamma_{i} \varepsilon$ für $i \in\{1,2,3\}$ und $\gamma_{i}=\varepsilon \gamma_{i-3} \varepsilon$ für $i \in\{7,8,9\}$ sowie $\gamma_{i}=\varepsilon \gamma_{i-9} \varepsilon$ für $i \geq 19$.

- Es gilt $\gamma_{i+1} R=\gamma_{i}$ für alle $i \not \equiv 0 \bmod 3$.

Exemplarisch für die Numerierung zeigt Figur 2.4 die Bereiche 13 bis 18 und 22 bis 27 mit dem Index der entsprechenden Transformation auf der Vierecksseite der entsprechenden Pyramide (abkürzend wird der Bereich $\gamma_{i} \mathcal{F}_{3}$ nur Bereich $i$ genannt).

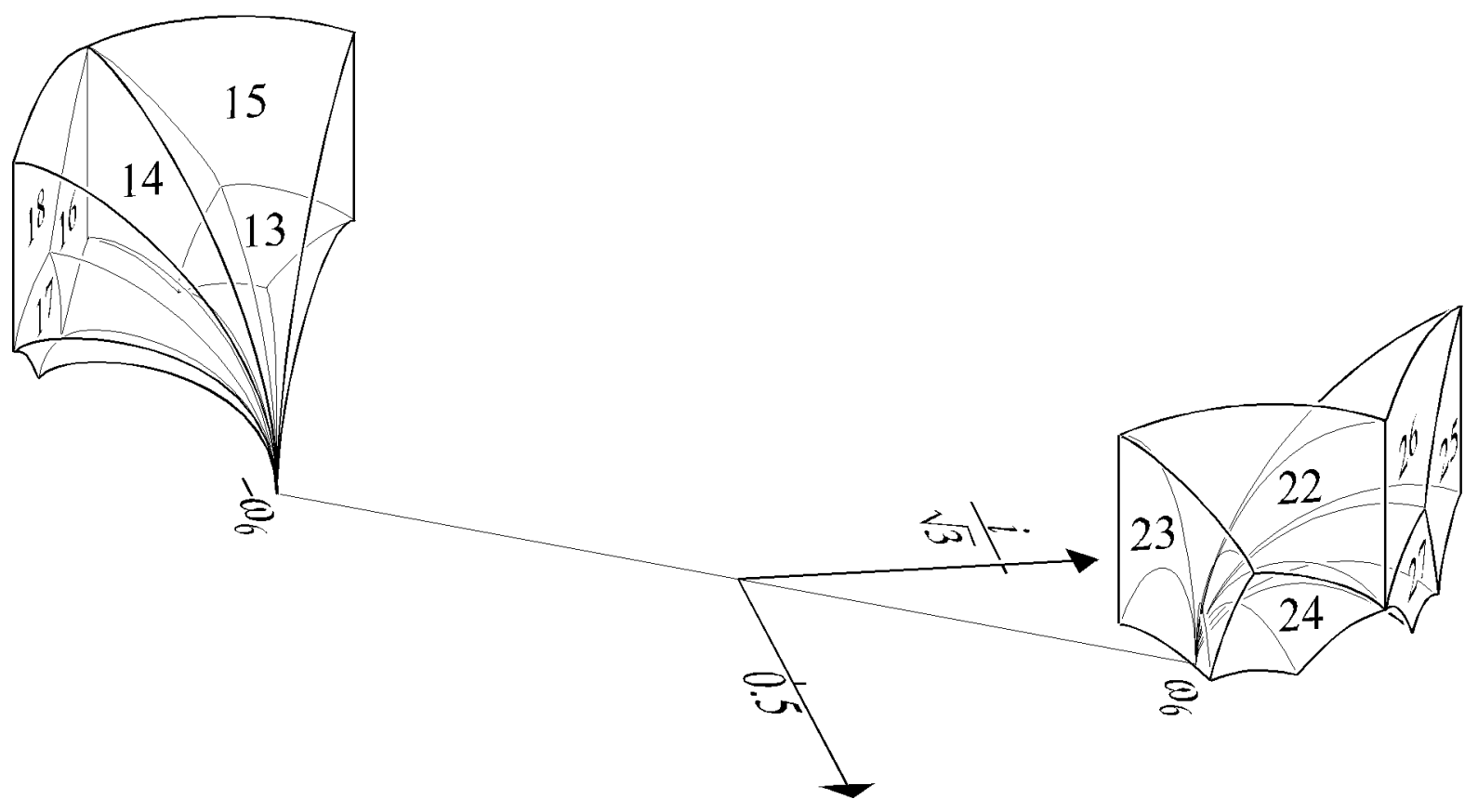

Abbildung 2.4: Die Numerierung der Bereiche 13-18 und 22-27

Der in Abbildung 2.3 dargestellte Bereich zeigt ein hyperbolisches Polyeder mit 41 Flächen, 68 Kanten und 29 Ecken, davon sind drei die (in $\tilde{\Gamma}$ nicht äquivalenten) Spitzen $\omega_{6},-\omega_{6}$ und $\infty$. Da die geometrischen Eigenschaften von $\tilde{\Gamma} \backslash \mathbb{H}^{3}$ einerseits für die Ideen der weiteren Berechnungen benötigt werden, andererseits aber auch an sich interessant sind, sollen sie an dieser Stelle weiter dargestellt werden.

Für zur Spitze $\infty$ gehörende Vertreter $\gamma, \gamma^{\prime}$ gilt für $(z, v) \in \gamma \stackrel{\circ}{\mathcal{F}_{3}}$, daß $\forall v^{\prime} \operatorname{mit}\left(z, v^{\prime}\right) \in \gamma^{\prime} \stackrel{\circ}{\mathcal{F}}_{3}$ folgt, daß $\gamma=\gamma^{\prime}$ gilt. Daher lassen sich die Bereiche zu $\infty$ ohne Überschneidung in die $z$-Ebene projizieren, wie bereits in den Figuren 2.1 und 2.3 angedeutet. Abbildung 2.5(a) zeigt eine solche 
Projektion für ein im Ursprung zentriertes Gebiet. Hierbei wird der Bereichsindex innerhalb der entsprechenden Projektionsfläche angegeben.

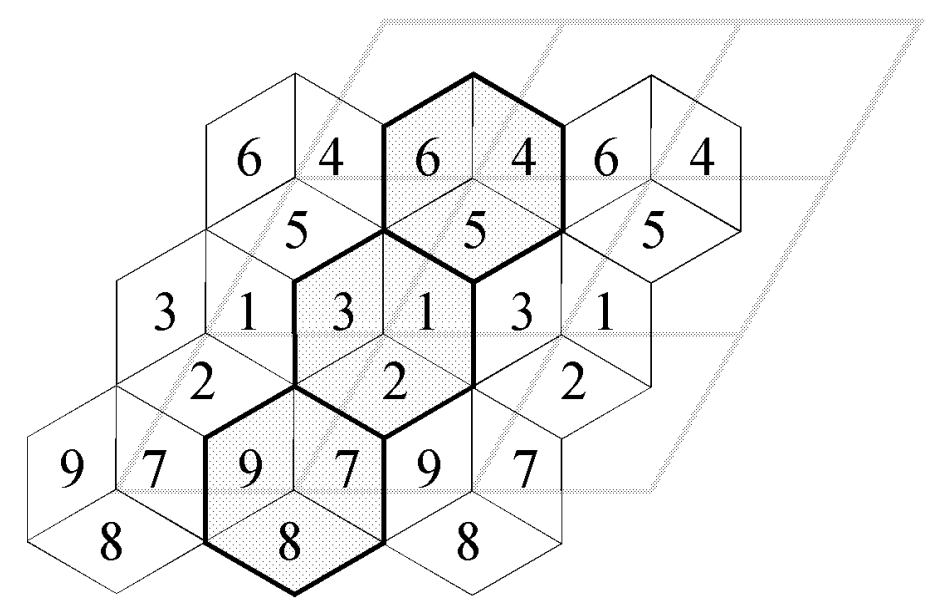

Abbildung 2.5: (a) Ausschnitt der Projektion der zu $\infty$ gehörenden Bereiche

Jeder Mittelpunkt eines der $3 \times 3$ Sechsecke liegt auf dem schattierten $\left(1, \omega_{6}\right)$-Gitter, die Sechsecke bilden so eine Flächenfüllung zu diesem Gitter, welche $2 \pi / 3$-drehungsinvariant ist. Da die Translationen $\left(\begin{array}{ll}1 & 3 \\ 0 & 1\end{array}\right)$ und $\left(\begin{array}{cc}1 & 3 \omega \\ 0 & 1\end{array}\right)$ aus der metaplektischen Gruppe sind, ist die Ebene entsprechend mit Kopien dieses Bereichs gefüllt. Tatsächlich sieht man, daß bereits ein $1 \times 3$-Bereich genügt, da $\left(\begin{array}{ll}1 & 1 \\ 0 & 1\end{array}\right) \in \tilde{\Gamma}$ gilt. Diese, zusammen mit der Verschiebung um $3 \omega$, erzeugt den Stabilisator von $\infty$, so daß neun nicht äquivalente Bereiche zu dieser Spitze gehören. Das dunkel unterlegte Gebiet stellt die für $\widetilde{\mathcal{F}}_{3}$ gewählten Bereiche dar.

Diese zweidimensionale Darstellung kann man auch für eine endliche Spitze $z_{0}$ wählen, denn diese läßt sich z. B. durch die automorphe Transformation

$$
\sigma_{z_{0}}=\left(\begin{array}{cc}
0 & -1 \\
1 & -z_{0}
\end{array}\right):(z, v) \mapsto\left(\frac{-\left(\overline{z-z_{0}}\right)}{\left|z-z_{0}\right|^{2}+v^{2}}, \frac{v}{\left|z-z_{0}\right|^{2}+v^{2}}\right)
$$

nach $\infty$ bringen. Für zu $\infty$ äquivalente $z_{0}$ erhält man so vergleichbare Bilder, die Abbildungen 2.5(b) und (c) stellen die Projektion für die Spitzen $\omega_{6}$ und $-\omega_{6}$ dar (also von $\sigma_{ \pm \omega_{6}} \gamma_{i} \mathcal{F}_{3}$ ), wobei der mittlere Bereich im Ursprung genau der „über” der Spitze liegende ist. Ein „Drahtgestell" aller neun hyperbolischen Urbilder dieser Sechsecke zeigt Abbildung 2.6.

Auch in den Spitzen $\pm \omega_{6}$ ist eine "Verschiebung" um $3 n$ metaplektisch, da $\sigma_{ \pm \omega_{6}}\left(\begin{array}{cc}1 & 3 n \\ 0 & 1\end{array}\right) \in$ $\tilde{\Gamma}, \forall n \in \mathcal{O}$, vgl. (2.18), wobei keine Translation geringerer Periode metaplektisch ist. Allerdings 

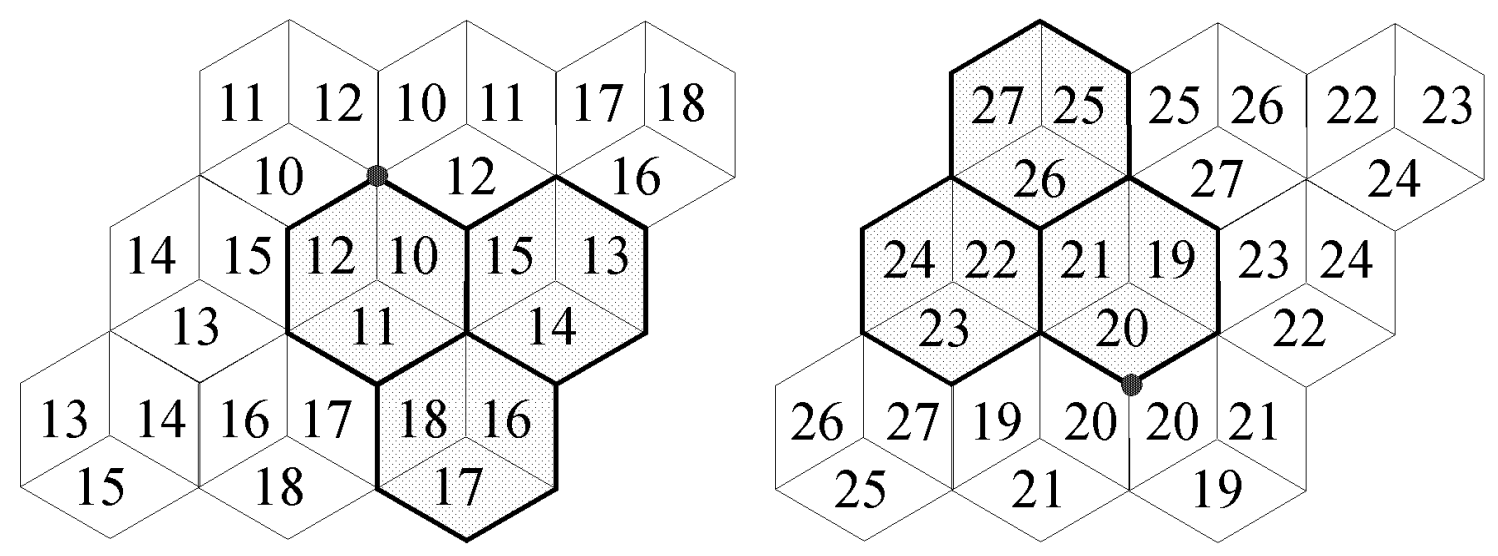

Abbildung 2.5: (b), (c) Ausschnitt der Projektion der zu $-\omega_{6}$ und $\omega_{6}$ gehörenden Bereiche

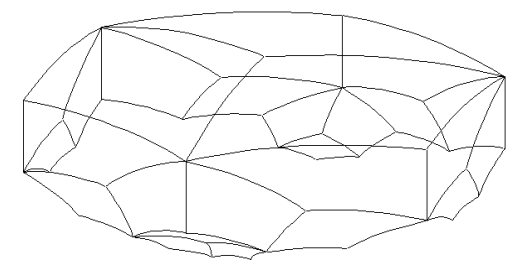

Abbildung 2.6: „Drahtgestell” zu Abbildung 2.5 (b), (c)

halten die metaplektischen Drehungen $\left(\begin{array}{cc}1 & -1 \\ 1 & 0\end{array}\right)$ bzw. $\left(\begin{array}{cc}1 & 1 \\ -1 & 0\end{array}\right)$ das entsprechende Gitter fest und drehen dieses um $2 \pi / 3$. Die Mittelpunkte dieser Transformationen sind durch kleine Kreise in den Abbildungen 2.5 (b) bzw. (c) markiert. Diese Drehungen, zusammen mit den beiden Translationen um 3 und $3 \omega$, erzeugen den Stabilisator von $\omega_{6}$ bzw. $-\omega_{6}$, dementsprechend sind auch hier neun nicht äquivalente Bereiche in einer Spitze zu finden, von denen die zu $\widetilde{\mathcal{F}}_{3}$ gehörenden wieder unterlegt dargestellt sind. Für Details hierzu siehe Kapitel 4.1.

Da die Abbildungen 2.5 das Nachbarschaftsverhalten von der metaplektischen Gruppe entlang der Dreiecksseiten dokumentieren, entsteht die Frage nach einem kompletten Nachbarschaftsgraphen. Um einen solchen zu erhalten, fehlen nur noch die Übergänge von $\widetilde{\mathcal{F}}_{3}$ entlang der Vierecksseiten oder, ohne auf die Klasse bezüglich $R$ einzugehen, entlang der Kombinationen dreier solcher in einer Sechseckseite. Dazu genügt es die Sechseckseiten von $\widetilde{\mathcal{F}}_{3}$ zu betrachten, wobei die Bereiche 4-6 direkt mit 19-21 und 7-9 mit 10-12 benachbart sind. Offene Sechseckseiten hat $\widetilde{\mathcal{F}}_{3}$ an den Bereichen 1-3, welche durch $S$ mit sich selbst verbunden sind, an den Bereichen 16-18, welche durch $\left(\begin{array}{cc}1 & 3 \omega+3 \\ 0 & 1\end{array}\right)$ mit 25-27 verbunden sind (vgl. Figur 2.4), und 13-15 und 22-24, 
welche vermöge $\left(\begin{array}{cc}-1 & -2 \\ 1 & 1\end{array}\right)$ und $\left(\begin{array}{cc}-1 & 2 \\ -1 & 1\end{array}\right)$ jeweils zu sich selbst benachbart sind. Zusammengefaßt ergibt dies Abbildung 2.7.

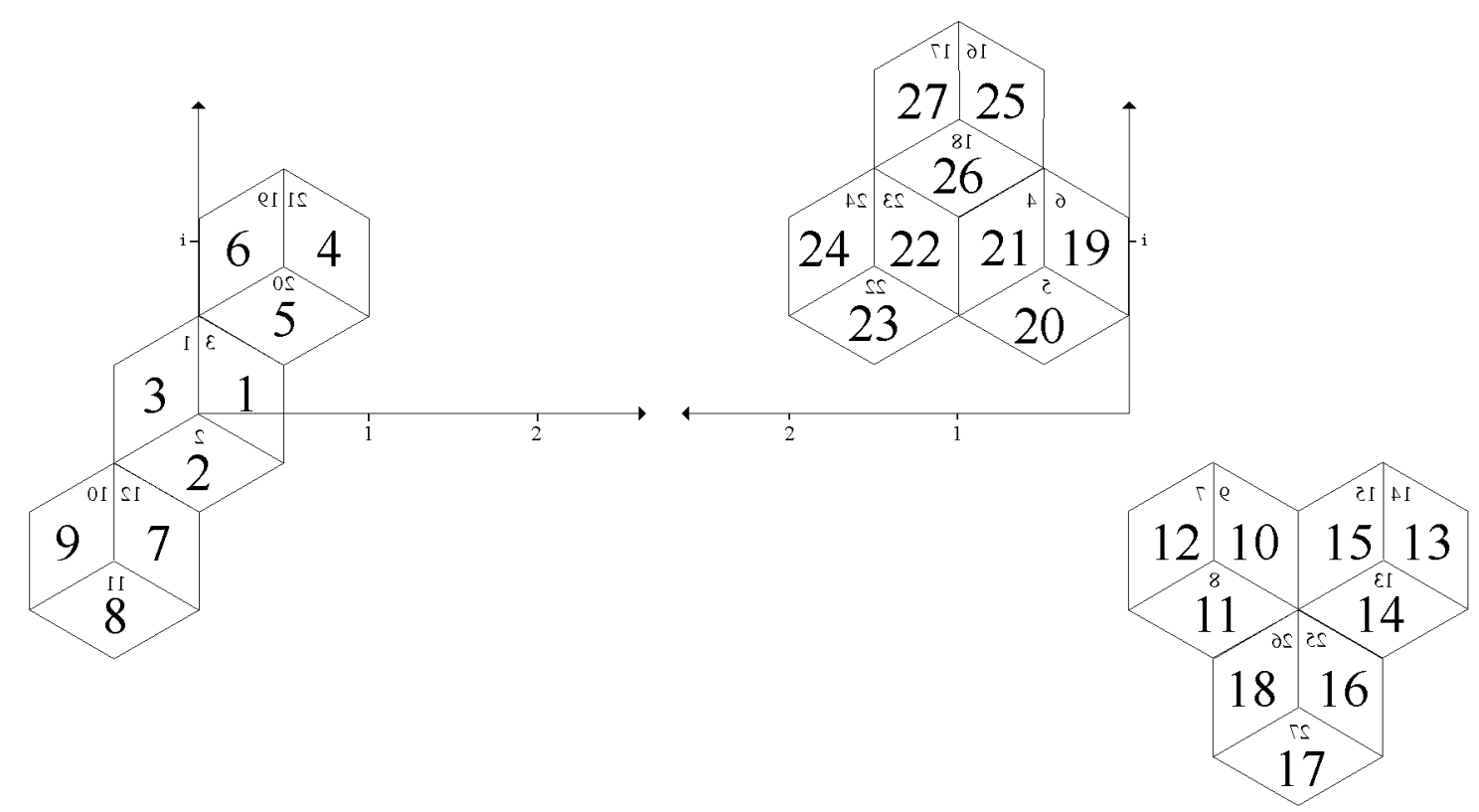

Abbildung 2.7: (a), (b) Die 27 Bereiche mit ihren Nachbarn (spiegelverkehrte Ziffern)

Abbildung 2.7 (a) zeigt die zur Spitze $\infty$ gehörenden Bereiche 1-9. Die Projektion ist Abbildung 2.5 (a) entnommen, die kleinen spiegelverkehrten Zahlen stellen die Bereiche auf der „Unterseite” der projizierten Flächen dar. Das eingezeichnete Koordinatensystem entspricht der Projektion, so daß der Mittelpunkt von dem zu 4, 5 und 6 gehörenden Sechseck der Punkt $\omega_{6}$ ist. Figur 2.7 (b) zeigt die zu den Spitzen $\pm \omega_{6}$ gehörenden Bereiche, dabei - genau wie bei (a) - aus der Spitze heraus gesehen, und ist daher aus den Figuren 2.5 (b) und (c) herauskopiert. Andererseits ist aber 2.7 (b) zum Teil gerade die „Unterseite” von 2.7 (a). Um dies darzustellen, wurde auch hier ein - nun linkshändiges - Koordinatensystem eingezeichnet, welches die Projektion aus den Spitzen zeigt (vgl. auch Figur 2.3).

Insgesamt erhält man aus den Abbildungen 2.5 und 2.7 den von der speziellen Wahl von $\left(\gamma_{i}\right)_{i}$ unabhängigen, in Figur 2.8 dargestellten Nachbarschaftsgraphen.

Zur Unterscheidung sind dabei die Vierecksnachbarschaften als doppelte Dreiecksnachbarschaften durch doppelte Linien, welche die Ziffern von unten erreichen, dargestellt. Die einfachen von oben kommenden Linien stellen in Abbildung 2.8 die Dreiecksnachbarschaften dar (Der Funda- 


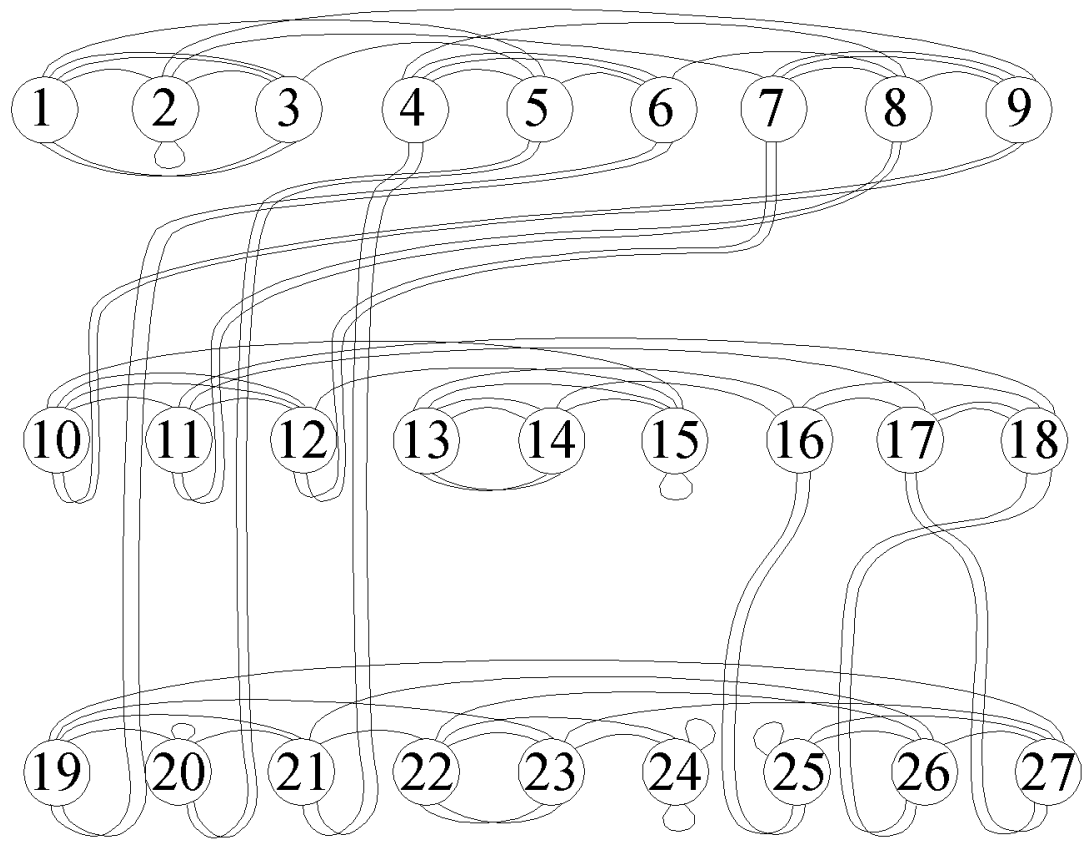

Abbildung 2.8: Der „Nachbarschaftsgraph” von $\tilde{\Gamma} \backslash \mathbb{H}^{3}$

mentalbereich $\mathcal{F}_{3}$ ist eine hyperbolische Pyramide mit einer viereckigen Grundseite, so daß jeder Bereich vier Nachbarn entlang einer Dreiecksseite und einen entlang einer Vierecksseite hat).

Alternativ zu der hier dargestellten Wahl des metaplektischen Fundamentalbereiches ist in [10] eine andere Wahl getroffen und in [15] übernommen worden. Diese soll abschließend kurz dargestellt werden. In [10] und [15] wurden 27 Vertreter $\gamma_{\nu}^{\prime}$ und $\bigcup_{\nu} \gamma_{\nu}^{\prime} \mathcal{F}_{3}$ als Fundamentalbereich gewählt. Die Numerierung der Bereiche folgt hier allerdings nicht Patterson, sondern wurde für

\begin{tabular}{|c|c|c|c|c|c|c|c|c|c|c|c|}
\hline$j / k$ & $\gamma_{j}^{\prime}$ & $j / k$ & $\gamma_{j}^{\prime}$ & $j / k$ & $\gamma_{j}^{\prime}$ & $j / k$ & $\gamma_{j}^{\prime}$ & $j / k$ & $\gamma_{j}^{\prime}$ & $j / k$ & $\gamma_{j}^{\prime}$ \\
\hline $1 / 1$ & $\left(\begin{array}{ll}1 & 0 \\
0 & 1\end{array}\right)$ & $6 / 8$ & $\left(\begin{array}{cc}\omega^{2} & -1 \\
0 & \omega\end{array}\right)$ & $10 / 10$ & $\sigma_{\omega^{2}}^{\prime}\left(\begin{array}{ll}1 & 0 \\
0 & 1\end{array}\right)$ & $15 / 17$ & $\sigma_{\omega^{2}}^{\prime}\left(\begin{array}{cc}1 & -1 \\
0 & 1\end{array}\right)$ & $19 / 19$ & $\sigma_{\omega}^{\prime}\left(\begin{array}{ll}1 & 0 \\
0 & 1\end{array}\right)$ & $24 / 25$ & $\sigma_{\omega}^{\prime}\left(\begin{array}{ll}1 & 1 \\
0 & 1\end{array}\right)$ \\
\hline $2 / 4$ & $\left(\begin{array}{cc}\omega & 0 \\
0 & \omega^{2}\end{array}\right)$ & $7 / 3$ & $\left(\begin{array}{cc}1 & -\omega \\
0 & 1\end{array}\right)$ & $11 / 13$ & $\sigma_{\omega^{2}}^{\prime}\left(\begin{array}{cc}1 & -\omega^{2} \\
0 & 1\end{array}\right)$ & $16 / 11$ & $\sigma_{\omega^{2}}^{\prime}\left(\begin{array}{cc}1 & -\omega \\
0 & 1\end{array}\right)$ & $20 / 24$ & $\sigma_{\omega}^{\prime}\left(\begin{array}{cc}1 & \omega^{2} \\
0 & 1\end{array}\right)$ & $25 / 21$ & $\sigma_{\omega}^{\prime}\left(\begin{array}{ll}1 & \omega \\
0 & 1\end{array}\right)$ \\
\hline $3 / 7$ & $\left(\begin{array}{cc}\omega^{2} & 0 \\
0 & \omega\end{array}\right)$ & $8 / 9$ & $\left(\begin{array}{cc}\omega & -1 \\
0 & \omega^{2}\end{array}\right)$ & $12 / 15$ & $\sigma_{\omega^{2}}^{\prime}\left(\begin{array}{ll}1 & \omega \\
0 & 1\end{array}\right)$ & $17 / 14$ & $\sigma_{\omega^{2}}^{\prime}\left(\begin{array}{cc}1 & \omega^{2} \\
0 & 1\end{array}\right)$ & $21 / 22$ & $\sigma_{\omega}^{\prime}\left(\begin{array}{cc}1 & -\omega \\
0 & 1\end{array}\right)$ & $26 / 23$ & $\sigma_{\omega}^{\prime}\left(\begin{array}{cc}1 & -\omega^{2} \\
0 & 1\end{array}\right)$ \\
\hline $4 / 2$ & $\left(\begin{array}{ll}1 & \omega \\
0 & 1\end{array}\right)$ & $9 / 6$ & $\left(\begin{array}{cc}\omega^{2} & 1 \\
0 & \omega\end{array}\right)$ & $13 / 12$ & $\sigma_{\omega^{2}}^{\prime}\left(\begin{array}{ll}1 & 1 \\
0 & 1\end{array}\right)$ & $18 / 18$ & $\sigma_{\omega^{2}}^{\prime}\left(\begin{array}{cc}1 & \omega-1 \\
0 & 1\end{array}\right)$ & $22 / 20$ & $\sigma_{\omega}^{\prime}\left(\begin{array}{cc}1 & -1 \\
0 & 1\end{array}\right)$ & $27 / 27$ & $\sigma_{\omega}^{\prime}\left(\begin{array}{cc}1 & \omega^{2}-1 \\
0 & 1\end{array}\right)$ \\
\hline $5 / 5$ & $\left(\begin{array}{cc}\omega & 1 \\
0 & \omega^{2}\end{array}\right)$ & & & $14 / 16$ & $\sigma_{\omega^{2}}^{\prime}\left(\begin{array}{cc}1 & 1-\omega \\
0 & 1\end{array}\right)$ & & & $23 / 26$ & $\sigma_{\omega}^{\prime}\left(\begin{array}{cc}1 & 1-\omega^{2} \\
0 & 1\end{array}\right.$ & & \\
\hline
\end{tabular}

Tabelle 2.2: Repräsentanten für $\Gamma / \tilde{\Gamma}$ mit Numerierung $k$ nach $[10]$ und $\sigma_{z_{0}}^{\prime}=\left(\begin{array}{cc}1 & 0 \\ z_{0} & 1\end{array}\right)$ 
die Vergleichbarkeit mit dem Vorangegangenen leicht umnumeriert, so daß $\forall \nu \exists \gamma \in \tilde{\Gamma}: \gamma \gamma_{\nu}=\gamma_{\nu}^{\prime}$ gilt, also die metaplektisch äquivalenten Bereiche gleiche Ziffern tragen. Die Numerierung aus [10] findet sich in der Spalte $j / k$ als zweiter Eintrag. Die entsprechenden Bereiche zur Spitze $\infty$ zeigt Abbildung 2.9(a) als im Ursprung zentrierte Projektion. Wie bei Proskurin ist $\gamma_{\nu}^{\prime}$ für

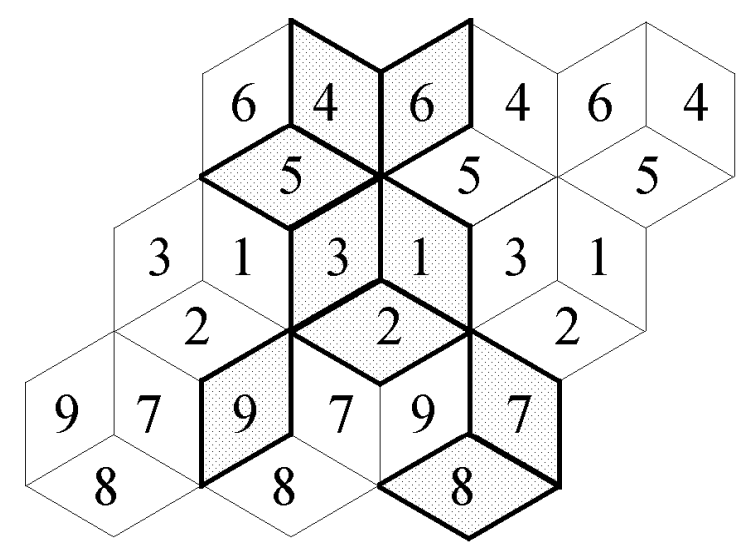

Abbildung 2.9: (a) Projektion der zu $\infty$ gehörenden Bereiche nach [10]

$\nu \geq 10$ in Tabelle 2.2 als Produkt von $\sigma_{z_{0}}^{\prime}=\left(\begin{array}{cc}1 & 0 \\ z_{0} & 1\end{array}\right)$ und einer Translation geschrieben worden, um deutlich zu machen, daß hier als endliche Spitzen $\omega$ und $\omega^{2}$ gewählt wurden. Projektionen für die Abbildungen 2.9(b) und (c) sind die Transformationen $\sigma_{\omega^{2}}$ und $R \sigma_{\omega}=\left(\begin{array}{cc}0 & -\omega \\ 1-\omega & -1\end{array}\right)$. Der
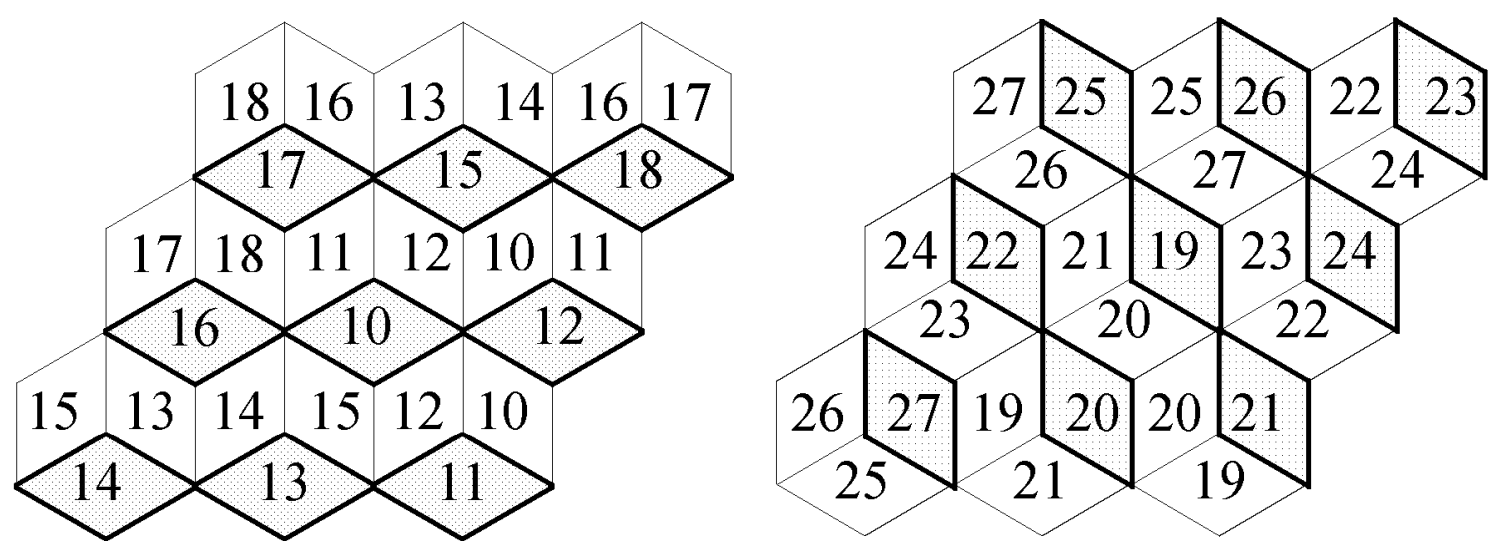

Abbildung 2.9: (b), (c) Projektion der zu $\omega^{2}$ und $\omega$ gehörenden Bereiche nach [10]

Mittelpunkt dieser Figuren ist $\omega$, da dies im wesentlichen dem Mittelpunkt der gewählten Bereiche entpricht. Da $\omega$ als Spitze zu $\omega_{6}$ äquivalent ist und $\omega^{2}=-\omega_{6}$ gilt, sind die Abbildungen 2.9 und 2.5 direkt vergleichbar. 


\subsection{Automorphe Formen auf $\tilde{\Gamma}$}

Die Situation auf $\tilde{\Gamma}$ (vgl. 2.17) ist der auf $\operatorname{PSL}(2, \mathrm{Z}[\omega])$ ähnlich, wobei für die metaplektische Überlagerung noch der Kubotacharakter eingeführt werden soll. Dieser Abschnitt beginnt aber mit einer Zusammenfassung einiger benötigter Fakten der kubischen Reziprozität, im wesentlichen nach [5].

Sei im folgenden immer $\pi$ eine Primzahl in Z $[\omega]$ und $\alpha \in \mathrm{Z}[\omega]$. Das kubische Restsymbol kann man am einfachsten mit einem Analogon der Euler-Formel definieren: Für alle $\pi$ mit $\mathrm{N}(\pi) \neq 3$ und alle $\alpha$ mit $\pi \nmid \alpha$ gilt die Definitionsgleichung:

$$
\alpha^{(\mathrm{N} \pi-1) / 3} \equiv(\alpha / \pi)_{3} \quad(\bmod \pi) \operatorname{mit}(\alpha / \pi)_{3} \in\left\{1, \omega, \omega^{2}\right\}
$$

Dies ist ein kubisches Restsymbol in dem Sinne, daß

$$
\begin{aligned}
(\alpha / \pi)_{3}=1 & \Leftrightarrow \quad \exists x: x^{3} \equiv \alpha \quad(\bmod \pi) \\
(\alpha \beta / \pi)_{3} & =(\alpha / \pi)_{3}(\beta / \pi)_{3} \\
\alpha \equiv \beta \quad(\bmod \pi) & \Rightarrow(\alpha / \pi)_{3}=(\beta / \pi)_{3}
\end{aligned}
$$

gilt. Um Primzahlen eindeutig zu charakterisieren, soll der Begriff einer primären Primzahl eingeführt werden: Es gibt genau eine zu $\pi$ Assoziierte mit $\pi \equiv 2+0 \omega \quad(\bmod 3)$. Diese soll primär heißen. Damit gilt die kubische Reziprozität:

Satz 1 (Kubisches Reziprozitätsgesetz) Seien $\pi_{1}$ und $\pi_{2}$ primär mit $\mathrm{N} \pi_{i} \neq 3$ und $\mathrm{N} \pi_{1} \neq$ $\mathrm{N} \pi_{2}$. Dann gilt:

$$
\left(\pi_{1} / \pi_{2}\right)_{3}=\left(\pi_{2} / \pi_{1}\right)_{3}
$$

und der

Ergänzungssatz Sei $\pi$ primär mit $\mathrm{N} \pi \equiv 1+3 a \quad(\bmod 9)$ und $\pi=3 m-1+3 b \omega, a, b, m \in \mathrm{Z}$. Dann gilt:

$$
(1-\omega / \pi)_{3}=\omega^{2 m}
$$

und

$$
(\omega / \pi)_{3}=\omega^{a} .
$$


Diese Aussagen können noch erweitert werden, für unsere Zwecke genügt dies jedoch bereits.

Den Kubotacharakter $\chi$ kann man für $\gamma=\left(\begin{array}{ll}a & b \\ c & d\end{array}\right) \in \Gamma(3)$ durch

$$
\chi(\gamma)= \begin{cases}(c / a)_{3}, & \text { falls } c \neq 0 \\ 1, & \text { falls } c=0\end{cases}
$$

direkt definieren, er ist mit dieser Definition ein Charakter auf $\Gamma(3)$. Außerdem ist

$$
\chi(\gamma)=1, \forall \gamma \in \Gamma(3) \cap \Gamma \quad \text { und } \quad \chi\left(g \gamma g^{-1}\right)=\chi(\gamma), \forall \gamma \in \Gamma(3), g \in \Gamma
$$

[10]. Jedes Element aus $\tilde{\Gamma}$ läßt sich in der Form $g \gamma$ mit $g \in \Gamma, \gamma \in \Gamma(3)$ schreiben, und die Identität (2.22) zeigt, daß man $\chi$ durch die Definition $\chi(g \gamma)=\chi(\gamma)$ zu einem Charakter auf $\tilde{\Gamma}$ erweitern kann. Damit sollen nun automorphe Formen für die metaplektische Gruppe definiert werden.

Sei $\chi: \tilde{\Gamma} \rightarrow \mathbb{C}$ der Kubotacharakter, und $f: \mathbb{H}^{3} \rightarrow \mathbb{C}$ mit

$$
f(\gamma w)=\chi(\gamma) f(w) \quad \forall \gamma \in \tilde{\Gamma}, w \in \mathbb{H}^{3}
$$

und

$$
-\Delta f=\lambda f=\left(1+R^{2}\right) f
$$

und $f$ hat höchstens polynomiales Wachstum in den Spitzen. Diese Funktionen $f \in F$ bilden den Vektorraum der automorphen Formen der metaplektischen Gruppe $\tilde{\Gamma}$ oder kurz der metaplektischen Formen. Der Raum der bezüglich des Petersson Skalarprodukts quadratintegrierbaren metaplektischen Formen bildet einen endlich-dimensionalen Hilbertraum [4]. Einen Unterraum darin bilden die Spitzenformen $\mathcal{S}(\tilde{\Gamma})$, also die Formen, welche in allen Spitzen verschwinden. Präziser formuliert: Sei $f$ eine metaplektische Form, dann gibt es, wie in Kapitel 2.2 beschrieben, eine Fourierentwicklung der Form

$$
f(z, v)=c_{0}(v)+\sum_{n \in \mathcal{O}}^{\prime} c(n) v K_{i R}\left(\frac{2 \pi}{A}|n| v\right) e\left(\frac{\langle i n, z\rangle}{A}\right) .
$$

Für Spitzenformen gilt $c_{0}(v)=0$ und auch für $\sigma \in \mathrm{PSL}_{2}(\mathrm{Z}[\omega])$ gilt mit

$$
f(\sigma(z, v))=c_{0}^{(\sigma)}(v)+\sum_{n \in \Lambda}^{\prime} c^{(\sigma)}(n) v K_{i R}\left(\frac{2 \pi}{A}|n| v\right) e\left(\frac{\langle i n, z\rangle}{A}\right)
$$


auch $c_{0}^{(\sigma)}(v)=0$. Diese zweite Entwicklung von $f$ heißt Fourierentwicklung zur Spitze $z_{0}$ falls $z_{0}=\sigma(\infty)$. Diese Schreibweise ist sinnvoll, da die Entwicklung nur von $z_{0}$, genauer im wesentlichen sogar nur von der Äquivalenzklasse von $z_{0}$ abhängig ist, denn eine Transformation, welche $z_{0}$ in eine äquivalente Spitze transformiert, ändert eine metaplektische Form höchstens um einen Charakter. Dementsprechend gibt es zu einer Spitzenäquivalenzklasse nur drei $c(n)$, welche sich lediglich um eine dritte Einheitswurzel unterscheiden. Eine elementare Art metaplektische Spitzenformen zu beschreiben ist dementsprechend die Betrachtung der Fourierkoeffizienten $c^{\left(z_{0}\right)}(n)$. Eine Form ist dabei bereits durch die Fourierdarstellung in einer beliebigen Spitze eindeutig beschrieben, da durch das exponentielle Verschwinden der $K_{i R}$ eine Konvergenz für beliebiges positives $r$ garantiert wird, aber die Güte der Konvergenz hängt offenbar von der Größe von $r$ ab. Auch aus diesem Grund scheint es angemessen zur Beschreibung einer Form nicht nur die Fourierentwicklung in der unendlichen Spitze zu betrachten, sondern auch die Fourierdarstellungen endlicher Spitzen mit einzubeziehen. Da sich hier die beiden endlichen Spitzen nur durch Vorzeichen unterscheiden, kann in diesem Fall die Entwicklung um $\omega_{6}$ direkt aus der um $-\omega_{6}$ hergeleitet werden und umgekehrt, so daß hier nur eine Fourierentwicklung $u m-\omega_{6}$ betrachtet wird.

Auch dieser Raum $\mathcal{S}(\tilde{\Gamma})$ der Spitzenformen zerlegt sich in eine orthogonale Summe, da der Laplace-Operator mit Drehungen und Spiegelungen in der $z$-Koordinate kommutiert. Es gibt aber nur noch eine unabhängige Bewegung, welche nicht in $\operatorname{PSL}(2, \mathrm{Z}[\omega])$ enthalten, und mit den Randbedingungen verträglich ist, darstellbar durch die Drehung $\varepsilon:(z, r) \mapsto(-z, r)$. Diese Symmetrie kann man allerdings ausnutzen, um die Fourierentwicklung zu vereinfachen, denn die metaplektischen Formen zerfallen dadurch in gerade und ungerade. Die im automorphen Fall auftauchende Bewegung $\bar{\varepsilon}$ operiert nicht in dem Raum der metaplektischen Formen.

Zuerst soll dazu die Fourierentwicklung genauer beschrieben werden. In der unendlichen Spitze gibt es eine Darstellung der Form

$$
f(z, v)=\sum_{\Lambda}^{\prime} a_{n} v K_{i R}\left(\frac{2 \pi}{A}|n| v\right) e\left(\frac{\langle n, z\rangle}{A}\right),
$$

wobei $\Lambda$ das zu $\mathcal{O}$ duale Gitter ist. Wenn man nun einsetzt, daß die metaplektischen Translationen gerade die Verschiebungen um 1 und $3 \omega$ sind und die zur Symmetrie $\varepsilon$ gehörenden Terme 
zusammenfaßt, erhält man einen Ansatz der Form

$$
f(z, v)=\sum_{n \in \mathcal{O}^{\infty}}^{\prime} c^{(\infty)}(n) v K_{i R}\left(\frac{2 \pi}{3 A}|n| v\right) \mathrm{cs}^{(\infty)}\left(2 \pi \frac{\langle i n, z\rangle}{3 A}\right),
$$

mit $\mathcal{O}^{\infty}=\mathcal{O} /\{ \pm 1\}$ und $\mathrm{cs}^{(\infty)} \equiv\left\{\begin{array}{l}\cos \\ \text { sin }\end{array}\right.$ je nachdem, ob $f$ gerade oder ungerade ist. Solche Umformungen sind für eine effiziente Numerik unumgänglich und bilden daher den Schwerpunkt von Kapitel 4. Für die endlichen Spitzen erhält man, wie oben angedeutet,

$$
f\left(\sigma_{\omega}(z, v)\right)=f\left(\sigma_{\omega_{6}}(z, v)\right)=\sum_{n \in \Lambda}^{\prime} c^{\left(\sigma_{\omega}\right)}(n) v K_{i R}\left(\frac{2 \pi}{A}|n| v\right) e\left(\frac{\langle i n, z\rangle}{A}\right)
$$

und damit

$$
c^{(\sigma-\omega)}(n)= \pm c^{\left(\sigma_{\omega}\right)}(-n)
$$

Die Hecketheorie dieser Funktionen ist komplizierter als im oben betrachteten automorphen Fall, da es nicht mehr für alle $n$, sondern nur noch in Spezialfällen Heckeoperatoren gibt (vgl. etwa [11]). Dementsprechend genügen die Fourierkoeffizienten keiner allgemeinen Multiplikativität mehr, und man kann nicht kanonisch $c(1)=1$ normieren. Allerdings werden die Realitätsbedingungen später so gewählt, daß die $c(n)$ für reelles $n$ je nach Parität reell oder imaginär sind. Des weiteren verschwindet $c(1)$ nicht für die ersten $f$, so daß hier die Normierungsbedingung $c(1)=1$ oder $c(1)=i$ gewählt werden konnte. 


\section{Kapitel 3}

\section{Eigenwerte von $\Delta$ auf der Bianchigruppe zu $D=-3$}

Eine wichtige Frage zum Verständnis des Laplace-Operators ist die Bestimmung des diskreten Teils des Spektrums. Um geeignete Hypothesen über die Natur des Spektrums zu erhalten, erscheint es sinnvoll und angemessen, mit Hilfe von numerischen Verfahren Näherungen zu Eigenwerten und entsprechenden Spitzenformen zu bekommen. Während der Schwerpunkt dieser Arbeit die Bestimmung von Eigenfunktionen zur metaplektischen Gruppe sein soll, ist es aus mehreren Gründen erforderlich, die Eigenwerte und die Eigenfunktionen des Laplace-Operators auf der automorphen Gruppe zu kennen. Einerseits ist dies darin begründet, daß die Eigenwerte für den metaplektischen Fall durch die kubische Shimura Korrespondenz im Zusammenhang mit den zum automorphen Fall gehörenden Eigenwerten stehen, andererseits sollen Hypothesen zum Zusammenhang von metaplektischen und automorphen Lösungen überprüft werden.

Für den genannten Fall der Bianchigruppe zu $D=-3$ gibt es bereits zwei interessante numerische Arbeiten: Zum einen die Studie [2] von Grunewald und Huntebrinker, welche die Laplacegleichung mit Hilfe finiter Elemente zu lösen sucht, und zum anderen die Arbeit [19] von Steil, welche das Problem durch Iteration der Heckegleichungen löst. Die Arbeit von Steil scheint numerisch genauer zu sein, aber um Werte mit hoher Sicherheit zu erhalten und um allgemeinere Methoden zu prüfen, sollen hier die Eigenwerte für diese automorphe Gruppe noch einmal bestimmt werden. Dabei erhält man dann auch die Fourierkoeffizienten für diese Entwicklung, welche für weitere Analysen von Interesse sind. Hauptergebnis dieser Bemühung ist die Bestätigung der Ergebnisse aus [19] in vollem Umfang (mit Ausnahme eines kleinen Fehlers 
von $\approx 3 \times 10^{-6}$ bei $R_{1}^{-}$) sowie die Gültigkeit des Verfahrens der Kollokation in diesem Fall.

\subsection{Numerische Bestimmung der Eigenfunktionen}

Gesucht ist eine nicht verschwindende Funktion $f \in L^{2}\left(\Gamma \backslash \mathbb{H}^{3}\right)$ mit $-\Delta f-\left(1+R^{2}\right) f=0$ und $\Gamma=\operatorname{PSL}(2, Z[\omega])$, welche in den Spitzen verschwindet. Wie in 2.2 ausgeführt, ist dies gleichbedeutend mit $f$ quadratintegrierbar auf $\mathbb{H}^{3}$ und

$$
f(z, r)=\sum_{n \in \mathcal{O} / \mathcal{O}^{\times}}^{\prime} c(n) r K_{i R}\left(\frac{2 \pi}{A}|n| r\right) \operatorname{cs}(n, z),
$$

und $\gamma f=f, \forall \gamma \in \Gamma$, vgl. $\left(2.11^{\prime}\right)$. An dem Ansatz (3.1) von $f$ erkennt man, daß sich die Bestimmung von $f$ auf die Bestimmung der Fourierkoeffizienten $c(n)$ reduziert. Steil löst dies durch Anwendung der Heckeoperatoren $T_{p}$ auf die Fourierreihe: Da die Fourierkoeffizienten durch die Heckerelationen bereits durch ihre Werte an den Primstellen definiert sind, genügt es die $c(p)$ zu bestimmen. Nach der Normierung $c(1)=1$ kann man die Gleichung $T_{p} f=c(\bar{p}) f$, explizit

$$
\frac{1}{|p|} \sum_{b \bmod (p)} f\left(\frac{z+b}{p}, \frac{r}{|p|}\right)+\frac{1}{|p|} f(p z,|p| r)=c(\bar{p}) f(z, r)
$$

ausnutzen, indem man die Punkte $((z+b) / p, r /|p|)$ und $(p z,|p| r)$, an denen $f$ ausgewertet wird, automorph in den zu $\infty$ gehörenden Fundamentalbereich bringt und dort $f$ durch eine endliche Teilsumme der Fourierreihe annähert (Die automorphe Transformation ist hier nötig, da die Fourierreihe am besten für großes $r$ konvergiert, denn $K_{i R}(x)$ verschwindet exponentiell für großes $x$ ). Dadurch erhält man ein homogenes nichtlineares Gleichungssystem, welches für Eigenwerte von $\Delta$ nichttriviale Lösungen besitzt, die sich durch die Inhomogenität $c(1)=1$ finden lassen. Numerische Lösungen besitzt (3.2) für endliche Annäherungen von $f$ durch (3.1) mit unbekannten $c(n)$ auch für $R$, welches nicht zu Eigenwerten von $\Delta$ gehört. Um festzustellen, wo Eigenwerte liegen, setzt man dann Testfunktionen ein, welche für automorphe Formen verschwinden, etwa $f\left(w_{\nu}\right)-\gamma f\left(w_{\nu}\right)$ für geeignetes $\gamma$ und $w_{\nu}$.

Durch Einsetzen von (3.1) in (3.2) erhält man ein nichtlineares Gleichungssystem, welches sich mit der Idee von Stark aus [18], der bereits die Fourierentwicklung von Eigenfunktionen des Laplaceoperators zu PSL $(2, \mathrm{Z})$ mit der Iteration von

$$
c(\bar{p})=\frac{T_{p} f(z)}{f(z)}
$$


„polierte". Steil nutzte diese Idee, um direkt Eigenwerte zu finden: $f$ wird mit $c(1)=1$ und $c(p)$ gleichverteilt in $[-2.2,2.2]$ angesetzt, denn für normiertes $f$ sind fast alle $|c(p)|<2$, siehe [16]. Dann wird (3.2) iteriert und nach Vorzeichenwechseln von $f\left(w_{\nu}\right)-S f\left(w_{\nu}\right)$ als Funktion von $R$ für einige $w_{\nu}$ und verschiedene Startwerte gesucht. Die Nichtlinearität zwingt allerdings zu großer Sorgfalt bei dieser Suche, da weder a priori eine Konvergenz vorhergesagt werden kann, noch ein Fixpunkt der Iteration zwangsläufig mit einer automorphen Form korrespondiert. Um alle Eigenwerte zu finden, ist sowohl eine ausgeprägte Variation in den $w_{\nu}$, als auch Tests für viele gleichverteilte Ausgangskonfigurationen erforderlich. Die Geschwindigkeit der Konvergenz ist ebenfalls von Fall zu Fall sehr unterschiedlich und variiert von 10 bis zu 10000 Iterationsschritten bei einer Genauigkeit von $10^{-30}$. Um so mehr ist die Präzision von [19] hervorzuheben, welche in allen betrachteten Punkten mit den hier erzielten Resultaten übereinstimmt.

Eine Alternative zur Bestimmung der $c(n)$ und der Eigenwerte, welche ohne den Einsatz von Heckeoperatoren auskommt, ist der Ansatz nur auf Grundlage der Automorphieinvarianz $f=\gamma f, \gamma \in \Gamma$. Da $\Gamma$ von $T=\left(\begin{array}{ll}1 & 1 \\ 0 & 1\end{array}\right), R=\left(\begin{array}{cc}\omega^{2} & 0 \\ 0 & \omega\end{array}\right)$ und $S=\left(\begin{array}{cc}0 & 1 \\ -1 & 0\end{array}\right)$ erzeugt wird, genügt die Erfüllung der Gleichungen $f=T f, f=R f$ und $f=S f$. Die ersten beiden Identitäten werden vom Fourieransatz (3.1) durch die Wahl des Gitters automatisch erfüllt. Zu lösen bleibt also

$$
f(z, r)=S f(z, r)=f\left(\frac{-\bar{z}}{|z|^{2}+r^{2}}, \frac{r}{|z|^{2}+r^{2}}\right),
$$

und wenn man nun $f$ durch eine endliche Reihe $f_{N}$ mit $N$ Gliedern

$$
f_{N}(z, r)=\sum_{\substack{n \in \mathcal{O} / \mathcal{O}^{\times} \\|n|<M_{N}}}^{\prime} c(n) r K_{i R}\left(\frac{2 \pi}{A}|n| r\right) \operatorname{cs}(n, z) \operatorname{mit}\left|\left\{n \in \mathcal{O} / \mathcal{O}^{\times}:|n|<M_{N}\right\}\right|=N
$$

annähert, eine Punktfolge $\left(w_{\nu}\right)_{1 \leq \nu \leq N^{\prime}}=\left(\left(z_{\nu}, r_{\nu}\right)\right)_{1 \leq \nu \leq N^{\prime}}$ und die Funktionen $\psi$ und $\psi^{\prime}$ durch $\psi(w)=r K_{i R}\left(\frac{2 \pi}{A}|n| r\right) \operatorname{cs}(n, z)$ und $\psi^{\prime}(w)=\psi(S w)$ definiert, so ist

$$
\begin{aligned}
\left(f_{N}\left(z_{\nu}, r_{\nu}\right)-S f_{N}\left(z_{\nu}, r_{\nu}\right)\right. & =0)_{1 \leq \nu \leq N^{\prime}} \\
\Leftrightarrow \quad\left(\sum_{\substack{n \in \mathcal{O}^{\prime} / \mathcal{O}^{\times} \\
|n|<M_{N}}}^{\prime} c(n)\left(\psi\left(w_{\nu}\right)-\psi^{\prime}\left(w_{\nu}\right)\right)\right. & =0)_{1 \leq \nu \leq N^{\prime}}
\end{aligned}
$$

ein $N^{\prime}$-dimensionales homogenes lineares Gleichungssystem mit $R$ als Parameter in $N$ Unbekannten. Numerisch erscheint es günstiger, $N^{\prime}$ etwas größer als $N$ zu wählen und statt exakter 
Lösung an $N$ Punkten eine Minimierung des Fehlerquadrats an $N^{\prime}$ Punkten zu suchen. Für die gesuchten $R$ besitzt (3.1) nichttriviale Lösungen, so daß man mit von $N$ und $N^{\prime}$ abhängiger Genauigkeit dort auch Lösungen von (3.5) erwarten kann. Um diese zu finden, bietet es sich an, durch Hinzunahme der Gleichung $c(1)=1$ ein inhomogenes System für normierte Eigenformen zu erhalten und für die eindeutigen Lösungen dieses Systems dann nach Vorzeichenwechseln von $f_{N}-S f_{N}$ an einigen Testpunkten zu suchen, da $f_{N}$ reell ist. Es ist günstig, die Testpunkte in der Nähe der Grenzfläche von $\mathcal{F}_{3}$ und $R^{2} S \mathcal{F}_{3}$ zu wählen, da dort beide Reihen gut konvergieren. Auch für die Kollokationspunkte genügt eine Folge von Punkten in einer „dünnen Schicht” um diese Fläche, also Punkte der Form $1 \geq|(z, r)| \geq 1-\varepsilon$ mit $z$ in der Fundamentalzelle und einem angemessenen Abstand $\varepsilon$ von der Einheitskugel. Dies gilt, da eine Lösung $f$ der Fouriergleichung in einem Fundamentalbereich $\mathcal{F}$, die in die Bilder $\gamma \mathcal{F}$ stetig und stetig differenzierbar fortgesetzt ist, eine Lösung der Laplacegleichung auf $\mathbb{H}^{3}$ mit den Randbedingungen $f(z)=\gamma f(z)$ darstellt. Natürlich dürfen die Punkte nicht nur auf der Grenzfläche liegen, da dort die Gleichung entartet und somit keine stetige Differenzierbarkeit mehr gegeben wäre. Wenn man an dieser Stelle nicht den Aufwand betreiben will, noch die Normalableitung mit in die Kollokationsgleichungen zu übernehmen, ist eine Verteilung der Punkte in einem gewissen Abstand von der Einheitskugel angebracht, um die Matrix günstiger zu konditionieren. Numerisch hat sich für diesen Abstand $\varepsilon$ ein Wert von etwa $1 / 8$ bewährt.

Es stellt sich heraus, daß dieses Verfahren gut konvergiert und Lösungen ergibt, die mit denen von Steil sehr gut übereinstimmen. Wie oben angedeutet, kann man auch an dieser Stelle noch die Heckerelationen einsetzen und mit der Idee von Stark die $c(n)$ iterativ verbessern. Es stellt sich dabei heraus, daß die Koeffizienten, speziell für kleine und mittlere $n$, bereits vor diesem Schritt gut sind, sofern der Eigenwert sehr genau bestimmt wurde. Fehler des Parameters $R$ in der Größenordnung von $10^{-6}$ machen es bereits unmöglich, mehr als etwa 10 Fourierkoeffizienten zu ermitteln, während die Iteration eine wesentlich geringere Genauigkeit bei der Bestimmung der Eigenwerte erfordert.

Am günstigsten zur Bestimmung der Fourierkoeffizienten erscheint hier also ein Weg, welcher zuerst die Kollokation nutzt, um die Eigenwerte zu erhalten, und in einem zweiten Schritt mittels Iteration der Hecke-Gleichungen die Eigenfunktion möglichst genau zu bestimmen sucht. 


\subsection{Ausführung des Algorithmus}

Für die Bestimmung der Eigenwerte wurde eine Reihe von Testfunktionen gewählt, welche an den zu Eigenwerten gehörenden Werten von $R$ Nullstellen haben. Als natürliche Wahl erscheinen $\delta_{N, \nu}(R)=f_{N}\left(w_{\nu}\right)-S f_{N}\left(w_{\nu}\right)$, mit $f_{N}$, wie in (3.4) erläutert und Punkten $w_{\nu}$ auf der Grenze zwischen $\mathcal{F}_{3}$ und $R^{2} S \mathcal{F}_{3}$. Die folgenden Graphen stellen dann solche Funktionen $\delta_{N, \nu}$ für verschiedene $w_{\nu}$ und $N$ dar, wobei auf der horizontalen Achse $R$ aufgetragen und die Normierung durch $\max _{R \in[a, b]}\left(\left|\delta_{N, \nu}(R)\right|\right)=1$ bestimmt wurde, was dem Einfluß von äußeren Faktoren, wie etwa der Entfernung von $w_{\nu}$ zu gegebenen Gitterpunkten, Rechnung trägt. Das Intervall $[a, b]$ ist dabei die gesamte Darstellungsbreite, also [10,29.5] für gerade und [6.5,26.5] für ungerade Eigenfunktionen.

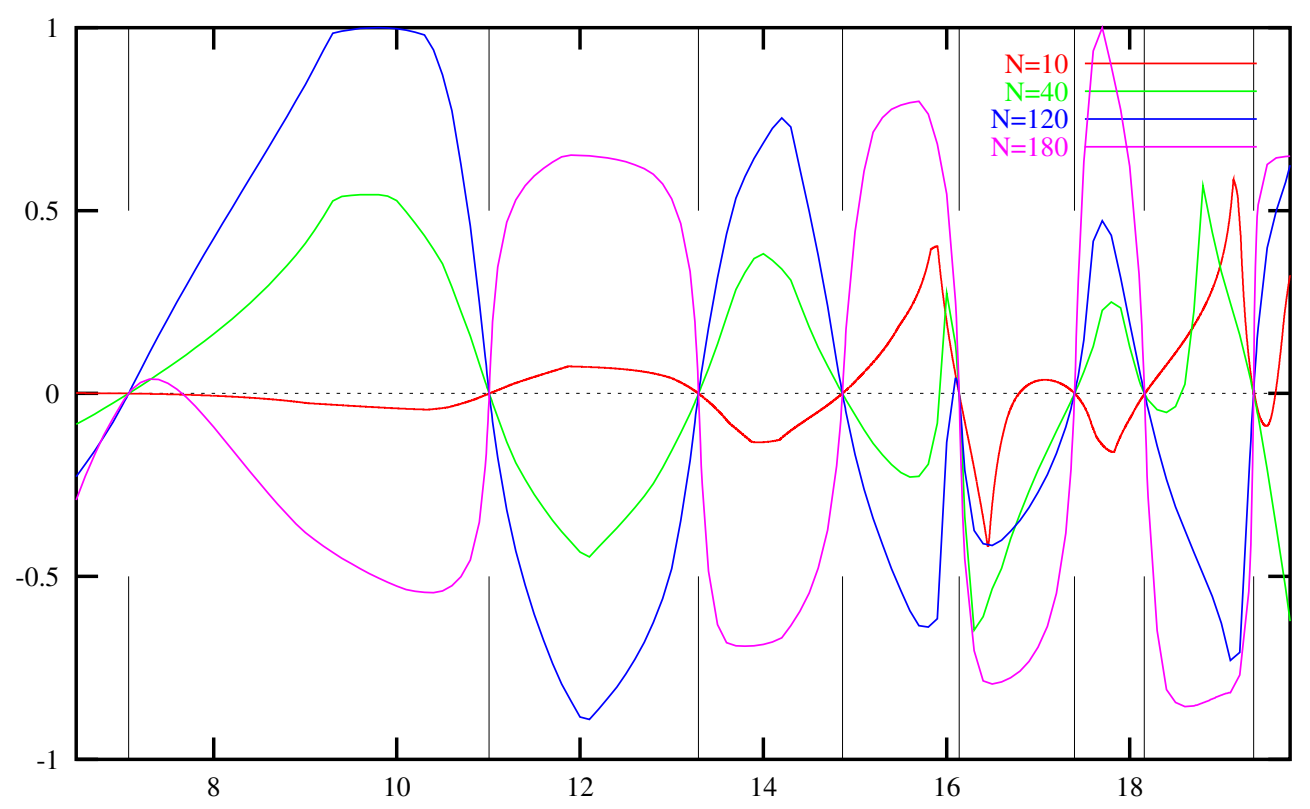

Abbildung 3.1: Die kleinsten 8 Eigenwerte der ungeraden Eigenfunktionen für verschiedene $N$

Als Eigenwerte wurden dann die Punkte identifiziert, an denen sämtliche Testfunktionen bei entsprechender Genauigkeit Nullstellen haben. Diese sind in den Abbildungen 3.1-3.8 durch lange senkrechte Striche an Ober- und Unterseite der Graphiken angedeutet. Nach der Bestimmung der Fourierkoeffizienten für ein gegebenes $N$ ist es wenig aufwendig, die $f_{N}\left(w_{\nu}\right)$ für viele $\nu$ zu bestimmen. Um alle Nullstellen zu finden, ist es allerdings bedeutend sicherer, auch für 
verschiedene $N$ eine Nullstellensuche vorzunehmen, da sich die Kurven für verschiedene $\nu$ weit mehr ähneln als für verschiedene $N$, wie die Abbildungen 3.2 und 3.3 exemplarisch an zwei sehr nahe beieinanderliegenden Nullstellen zeigen.

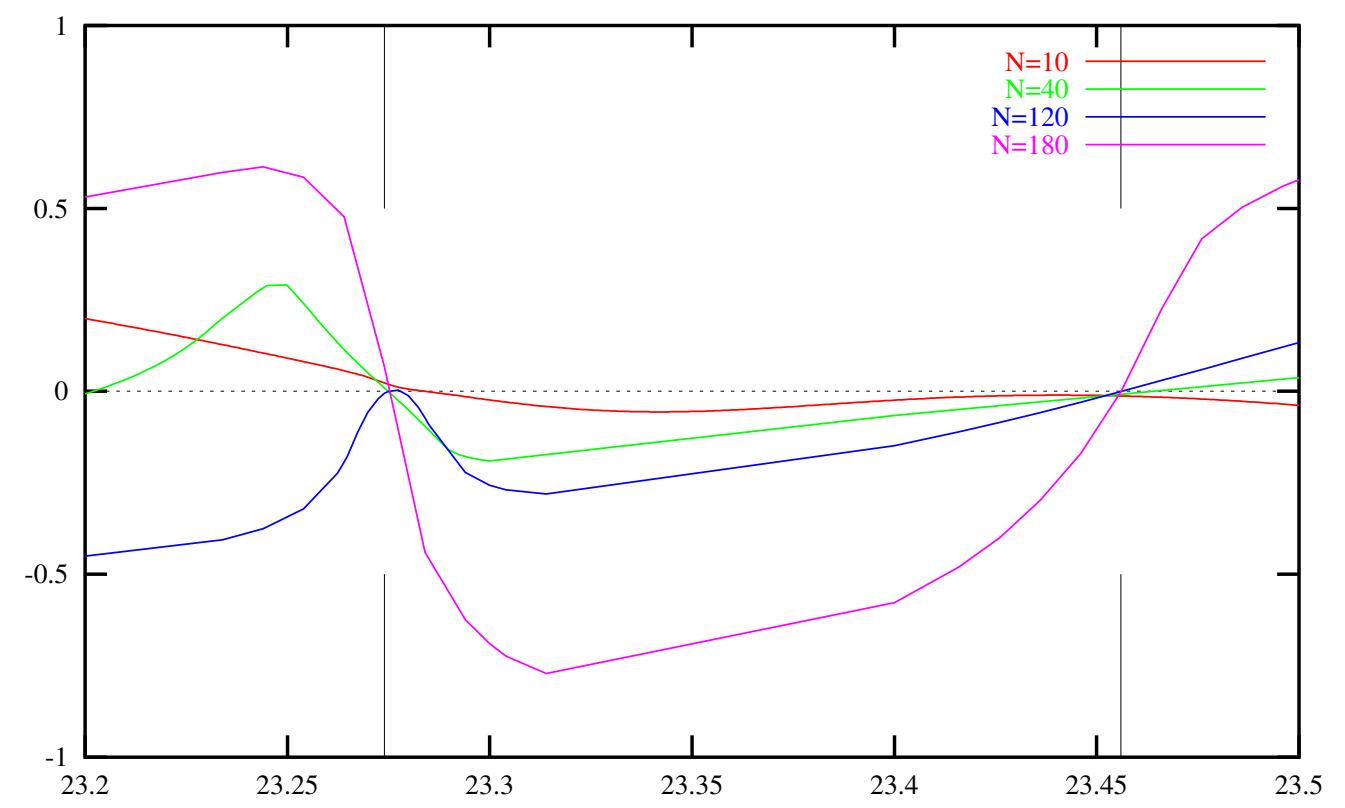

Abbildung 3.2: Die Funktionen $\delta_{N, \nu}$ im Bereich nahe $R_{13}^{-}$und $R_{14}^{-}$für verschiedene $N$

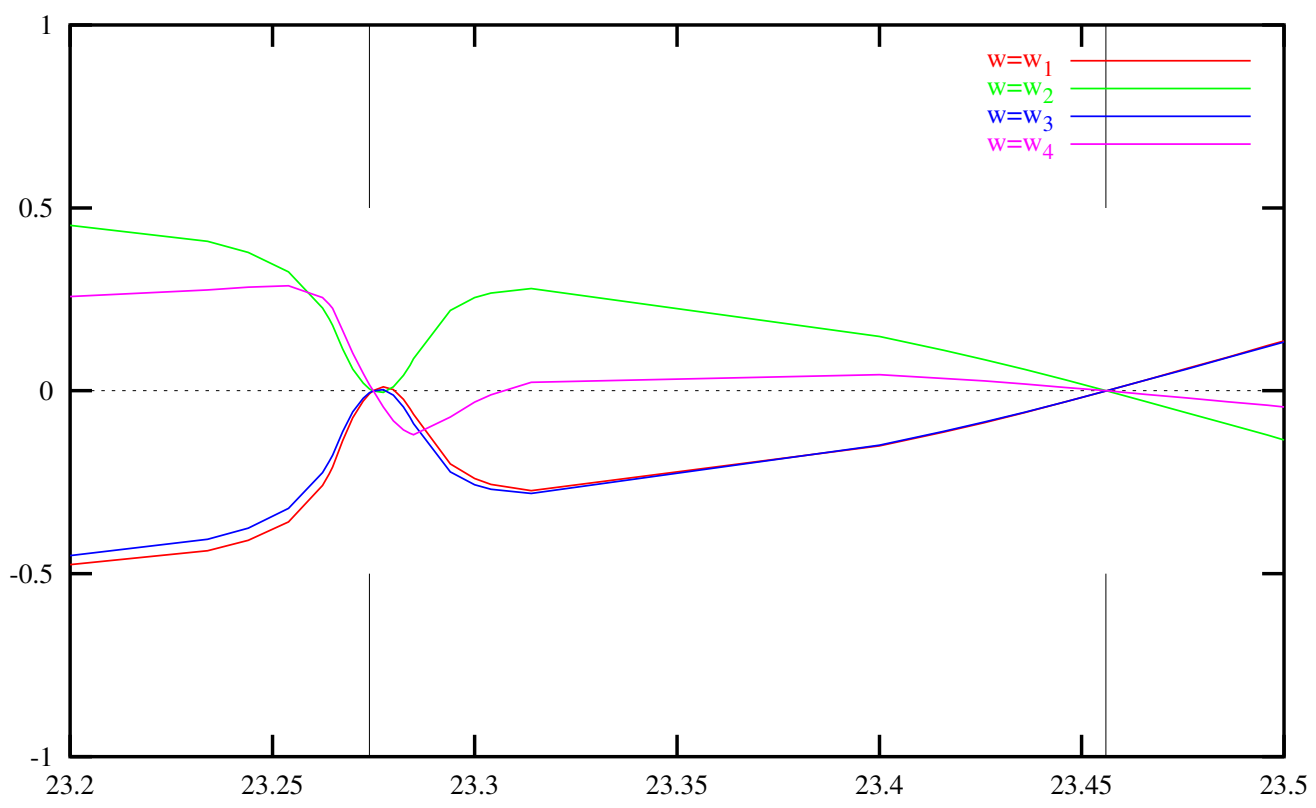

Abbildung 3.3: Wie Abbildung 3.2 für verschiedene $\nu$ bei $N=120$ 


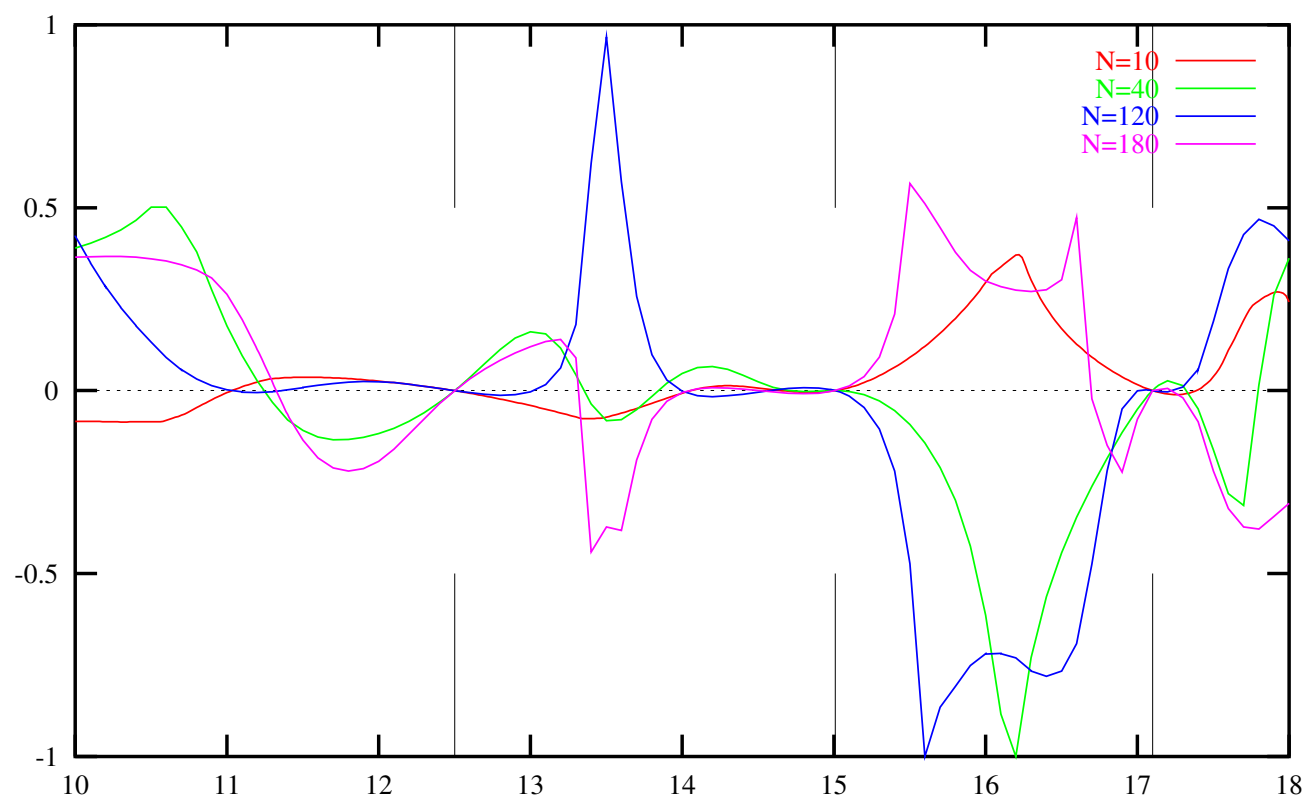

Abbildung 3.4: Wie 3.1 für die kleinsten drei Eigenwerte der geraden Eigenfunktionen

Die Eigenwerte der geraden Eigenfunktionen sind, wie erwartet, schwieriger zu bestimmen als im ungeraden Fall, wie man bereits an den $\delta_{N}$ bei den kleinsten drei Eigenwerten in Abbildung 3.4 erkennen kann. Es gibt hier sehr häufige Vorzeichenwechsel einzelner Funktionen, die sich teilweise nur bei genauer Bestimmung trennen lassen. Auch bei der Iteration der Hecke-Gleichungen ist eine Konvergenz bei ungeraden Funktionen bedeutend häufiger als bei geraden. Die Graphen der $\delta_{N}$ für größeres $R$ finden sich am Ende dieses Kapitels auf den Seiten 43-44.

Die Bestimmung der Nullstellen gelingt bereits mit einiger Genauigkeit bei $N=10$ und $N^{\prime}=15$ (also lediglich zu den kleinsten sechs Primstellen), wie auf den Abbildungen 3.1-3.6 zu erkennen ist. Allerdings verliert für derart kleine $N$ bei etwas größerem $R$ die Gleichung (3.5) ihre Gültigkeit als Annäherung an (3.1). Für ungerade Eigenfunktionen ist dies etwa bei $R \approx 26$ der Fall, für gerade bereits bei $R \approx 22$. Als Referenz ist der Fehler $\delta_{10, *}$ von $f_{10}$ auch in den Abbildungen 3.7-3.8 auf der Seite 44 mit angegeben, allerdings neu normiert, da $\delta_{10, *}$ hier um mehrere Größenordnungen größer ist als für $R<22$. Zur tatsächlichen Bestimmung der Eigenwerte wurden $\delta_{N, \nu}$ für $N \in\{40,80,120,180\}, N^{\prime}=N+40$ und einige $\nu$ ausgewertet und die Vorzeichenwechsel mit der Methode von van Wijngaarden, Dekker und Brent nach [14] zur Genauigkeit von $\approx 10^{-4}$ gesucht. Die gemeinsamen Wurzeln aller dieser Funktionen wurden 
dann mit hoher Genauigkeit noch einmal für $N \in\{280,290,300\}$ bestimmt. Diese sind in der Tabelle 3.1 wiedergegeben. Einige Werte sind nicht mit voller Genauigkeit bestimmt.

\begin{tabular}{|c|c|c|c|}
\hline$R_{1}^{+}$ & 12.5001001675229770430978354906802 & $R_{1}^{-}$ & 7.07200418587520500073719418672873 \\
\hline$R_{2}^{+}$ & 15.0097060709209126370976132530945 & $R_{2}^{-}$ & 11.0081133593531090316723794529573 \\
\hline$R_{3}^{+}$ & 17.0986557064180261592442699678523 & $R_{3}^{-}$ & 13.2931627112188984823094208008053 \\
\hline$R_{4}^{+}$ & 18.8245088146673522715806652306503 & $R_{4}^{-}$ & 14.8635983434437078868675448330041 \\
\hline$R_{5}^{+}$ & $\left.19.0673905227071151086884704718575^{\dagger}\right)$ & $R_{5}^{-}$ & $16.137241619682603042890466276032^{*)}$ \\
\hline$R_{6}^{+}$ & 21.0780050975078173447290628988758 & $R_{6}^{-}$ & 17.3966859129367773117760260840093 \\
\hline$R_{7}^{+}$ & 21.1522303568547858779272343156205 & $R_{7}^{-}$ & 18.1601386994023526127303635808520 \\
\hline$R_{8}^{+}$ & 22.6100896954135924148243402344010 & $R_{8}^{-}$ & 19.350972511450350770936863198 \\
\hline$R_{9}^{+}$ & $22.65485545419221836423760111 \quad *)$ & $R_{9}^{-}$ & 20.1789061256797550771187651363320 \\
\hline$R_{10}^{+}$ & 23.7728843978354428837879854302764 & $R_{10}^{-}$ & 20.9422402749763460850407177438543 \\
\hline$R_{11}^{+}$ & 24.3246702709349018260909886648456 & $R_{11}^{-}$ & 21.9331772500684208381726440179063 *) \\
\hline$R_{12}^{+}$ & $\left.24.3460166493593556990559023527910^{\dagger}\right)$ & $R_{12}^{-}$ & 22.2619272697554987218340899948337 \\
\hline$R_{13}^{+}$ & 25.7680039576173147834606262090925 & $R_{13}^{-}$ & $\left.23.2748864176344707128558201126477^{*}\right)$ \\
\hline$R_{14}^{+}$ & 26.0866661053171437948512455916694 & $R_{14}^{-}$ & 23.4560490320008575949252406736008 \\
\hline$R_{15}^{+}$ & $\left.26.2686130281005536217250009131105^{*}\right)$ & $R_{15}^{-}$ & 24.5033415221317239974125323832360 *) \\
\hline$R_{16}^{+}$ & 27.3801203053961765091777312579964 & $R_{16}^{-}$ & 25.2459697318605313119824876884217 \\
\hline$R_{17}^{+}$ & 27.5595027037814778884873465630354 & $R_{17}^{-}$ & 25.7148325569507826321180309299268 \\
\hline$R_{18}^{+}$ & 27.9946427548674785289001169369983 & $R_{18}^{-}$ & 26.1546423402881729189450686138513 \\
\hline$R_{19}^{+}$ & 28.7170190365196255597338851380743 & $R_{19}^{-}$ & $26.3006796482475459390159293872656^{*}$ \\
\hline$R_{20}^{+}$ & $29.0740531321902954346690576030950 *)$ & & \\
\hline$R_{21}^{+}$ & 29.0801011283508768049471045162841 & & \\
\hline$R_{22}^{+}$ & 29.2771928174903244174396417799349 & & \\
\hline
\end{tabular}

*) Diese Eigenwerte haben einen Eigenraum der Dimension 2.

$\dagger$ Diese Eigenwerte stammen von $\operatorname{PSL}(2, \mathrm{Z})$.

Tabelle 3.1: Liste der kleinsten Eigenwerte für die ersten 25 geraden und 25 ungeraden Eigenfunktionen. Die Eigenwerte $R_{\nu}^{+}$bezeichnen die Symmetrieklassen $D$ und $G$, die Eigenwerte $R_{\nu}^{-}$ die Symmetrieklassen $C$ und $H$.

In den Tabellen im Anhang auf CD finden sich die zu diesen Eigenfunktionen gehörenden Fourierkoeffizienten. Für alle errechneten Koeffizienten gilt $|c(p)|<2$. Da nicht für alle $R$ eine Iteration der Hecke-Koeffizienten durchgeführt wurde, hat diese Ungleichung für die nicht 
iterierten Koeffizienten nur für kleine und mittlere $p$ Gültigkeit.

In den Abbildungen 3.5-3.8 sind die $\delta_{N, \nu}$ für größere $R$ dargestellt, wobei der Deutlichkeit halber die Bestimmung der Symmetrieklassen $D$ und $G$ mehr Raum einnimmt.

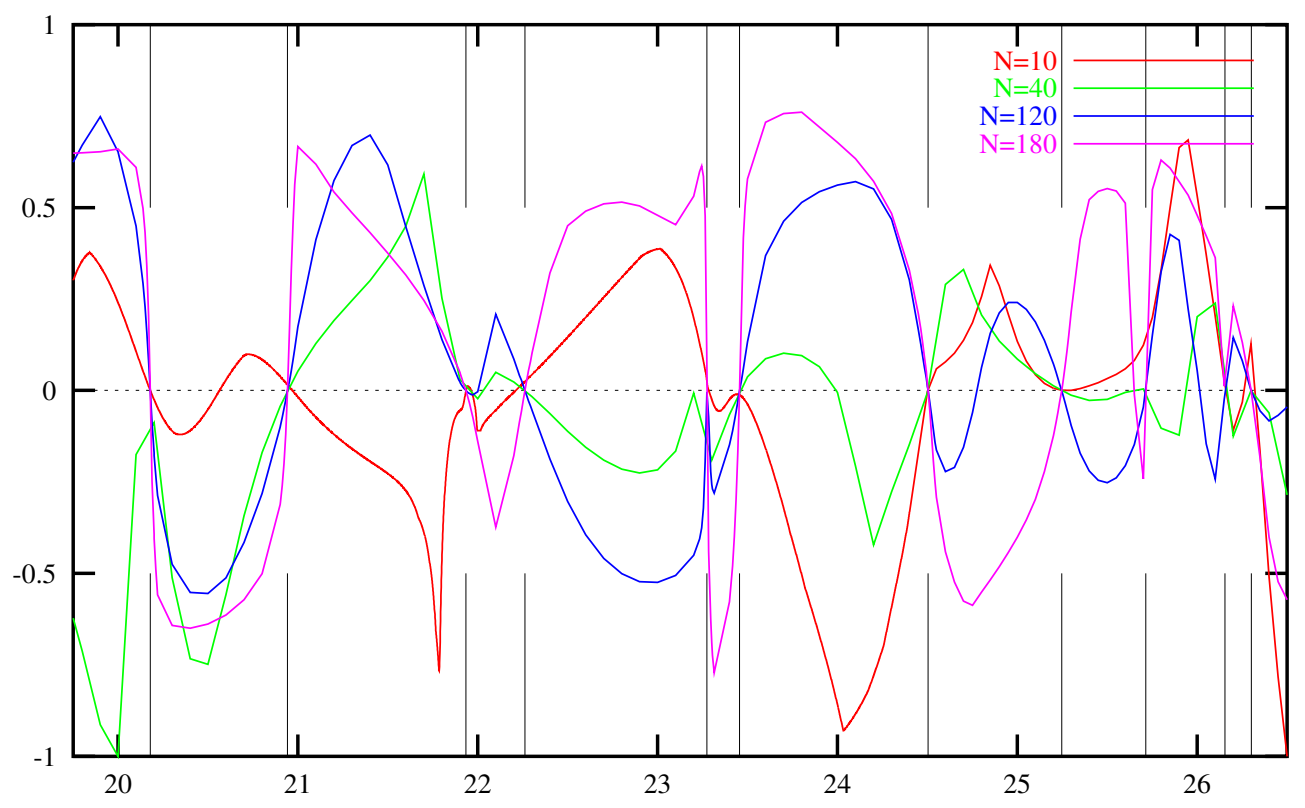

Abbildung 3.5: Die Eigenwerte $R_{9}^{-}-R_{19}^{-}$für verschiedene $N$

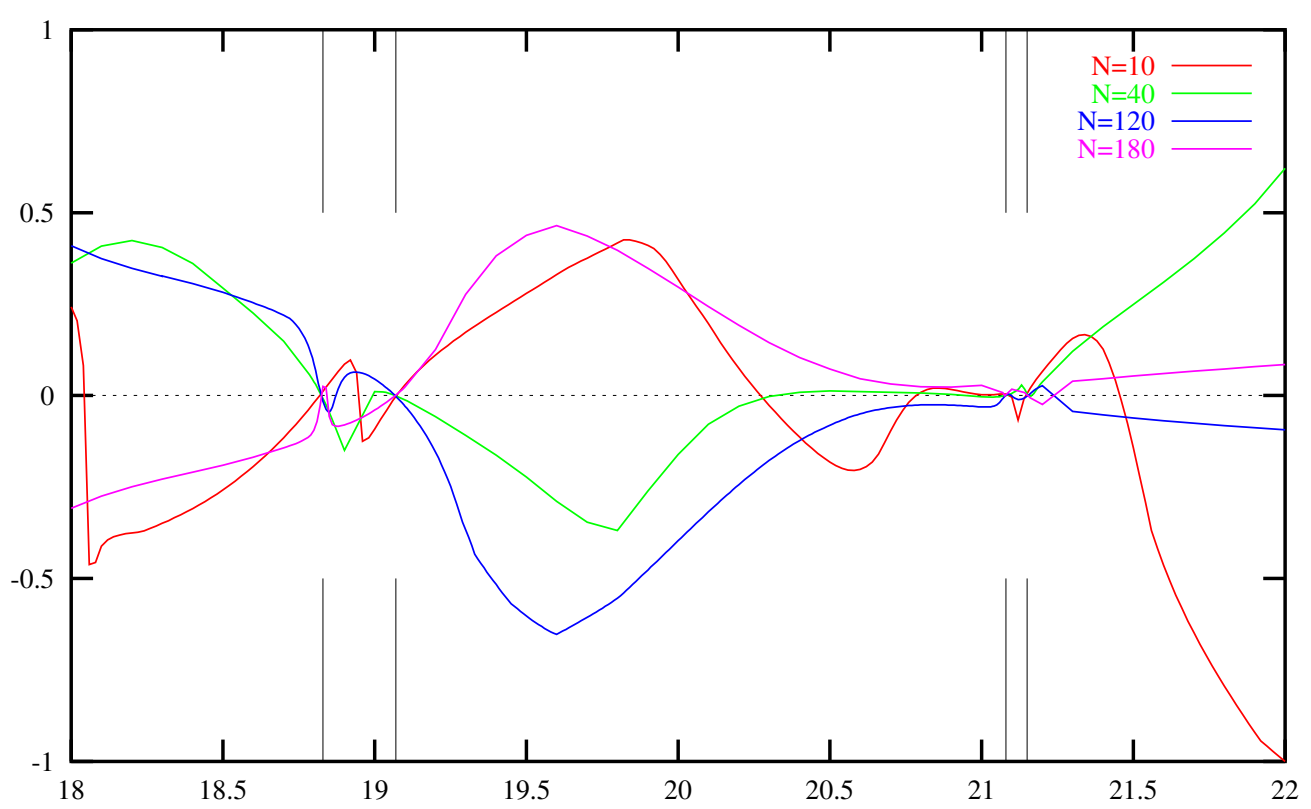

Abbildung 3.6: Wie Abbildung 3.4 für die Eigenwerte $R_{4}^{+}-R_{7}^{+}$ 


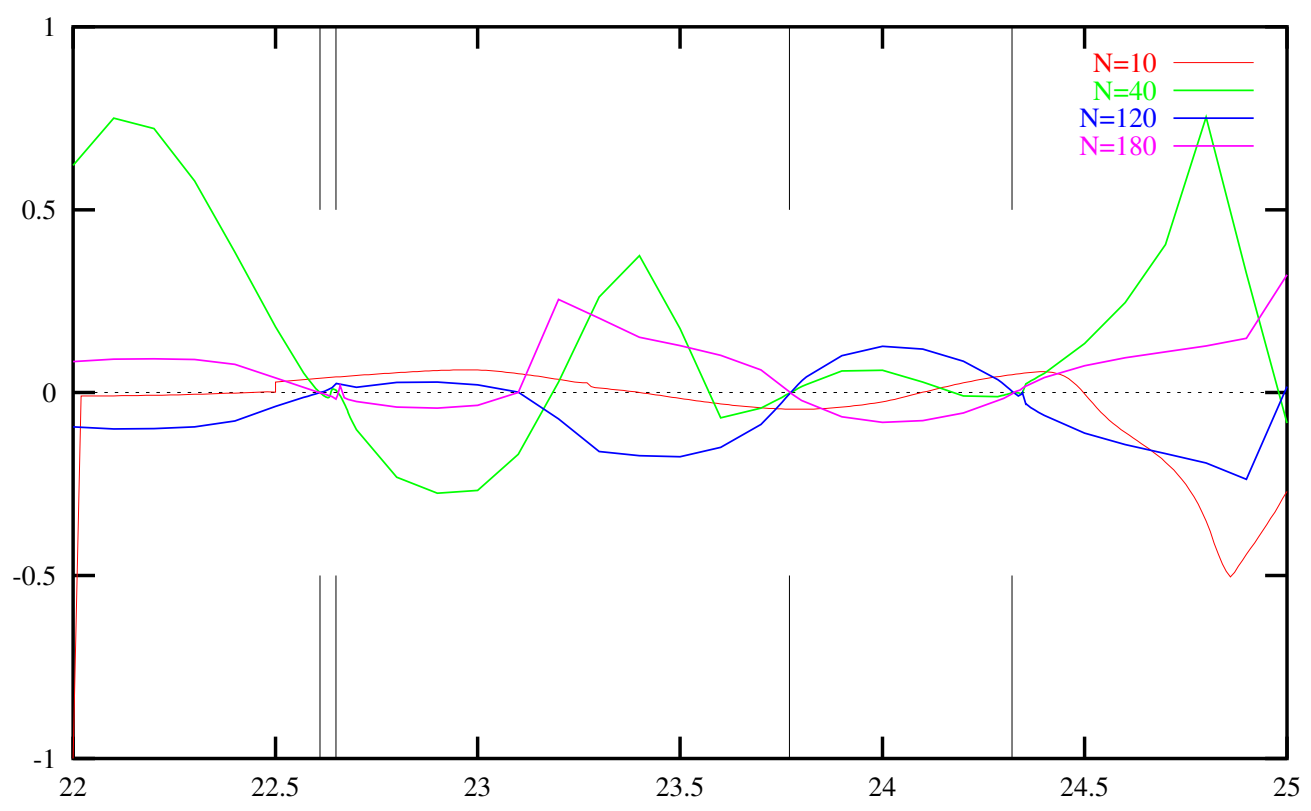

Abbildung 3.7: Wie Abbildung 3.4 für die Eigenwerte $R_{8}^{+}-R_{12}^{+}$

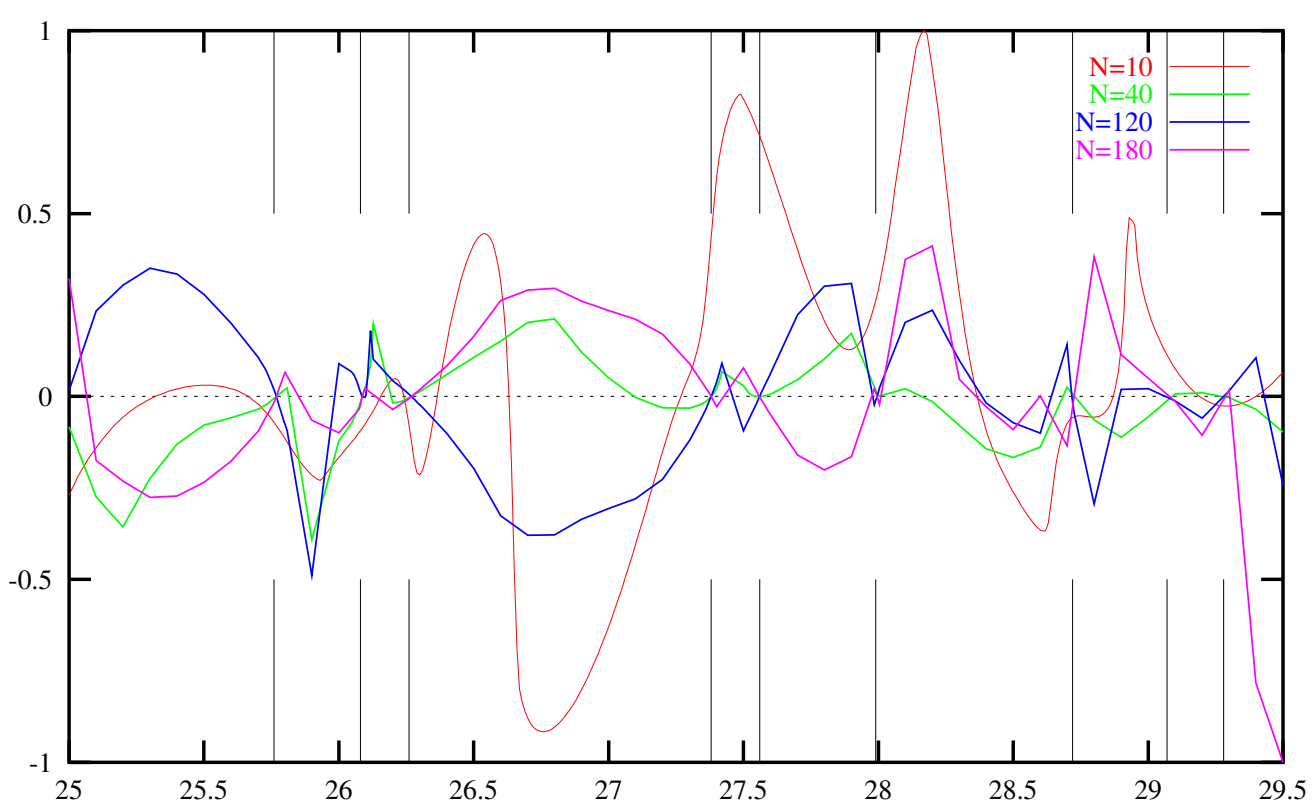

Abbildung 3.8: Wie Abbildung 3.4 für die Eigenwerte $R_{13}^{+}-R_{22}^{+}$

Abgeschlossen wird dieses Kapitel durch die Tabelle 3.2, in der die ersten 55 primen Fourierkoeffizienten einiger Eigenfunktionen angegeben sind. Für die Berechnung genügen hier prime Koeffizienten, da sich die $c(n)$ durch (2.15) aus den $c(p)$ bestimmen lassen. 


\begin{tabular}{|c|c|c|c|c|}
\hline$p$ & $R_{1}^{+} \approx 12.50010016752298$ & $R_{1}^{-} \approx 7.072004185875205$ & $R_{2}^{-} \approx 11.00811335935311$ & $R_{14}^{-} \approx 23.45604903200085$ \\
\hline 1 & 1.00000000000000000000 & 1.00000000000000000000 & 1.00000000000000000000 & 1.00000000000000000000 \\
\hline $2+\omega$ & & & -0.00000000000000000000 & -0.00000000000000000000 \\
\hline 2 & 1.20930465445456811193 & 0.42245559832595241254 & 1.66152188472286908857 & 1.60573144490655888126 \\
\hline $3+2 \omega$ & & 0.85350012153754368354 & -0.45918749523868322305 & \\
\hline $3+\omega$ & 0.39044333437748070153 & -0.85350012153754368354 & 0.45918749523868322305 & -0.42201628227931214702 \\
\hline $4+3 \omega$ & 0.89548986613779 & 0.04667951773193724842 & 0.89713784531043008091 & 1.42686536082801982926 \\
\hline $4+\omega$ & & -0.04667951773193724842 & -0.89713784531043008091 & -1.42686536082801982926 \\
\hline $5+3 \omega$ & 0.17205659485048099256 & 81701 & 5614496496 & -0.61374061060339160205 \\
\hline $5+2 \omega$ & & 2298981701 & -0.93633382095614496496 & \\
\hline 5 & & 1.45231746474063509409 & -0.48029997223666692059 & \\
\hline $6+5 \omega$ & 2294 & & 429 & -1.055 \\
\hline & & & 6429 & \\
\hline & & & & \\
\hline & & & 909669 & -0.573890581023 \\
\hline $7+6 \omega$ & -0.11378178176198370509 & 0.11730467647852126982 & 0.38699579564369867831 & 0.21610920617963434100 \\
\hline & & & & \\
\hline & & & & \\
\hline & & & & \\
\hline $9+7 \omega$ & -0.73438679172969239942 & 96157428 & 0914431 & -0.957757277398 \\
\hline $9+2 \omega$ & -0.73 & 428 & 1431 & \\
\hline & & & & \\
\hline & & & 00 & 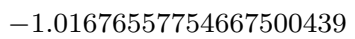 \\
\hline $10+7 \omega$ & 8701 & 2275 & & -1.4 \\
\hline & & & & \\
\hline & 1.2 & & 007 & 80 \\
\hline & & & & \\
\hline & & & 250 & -0.2 \\
\hline & & & & \\
\hline & -0.3 & & & \\
\hline $12+5 \omega$ & -0.31696511438511571181 & -0.92347287753052697576 & -0.27277185388202943333 & -1.240463056960 \\
\hline & & & & \\
\hline & & & & \\
\hline & & & & \\
\hline & & & & -0.97 \\
\hline & 0.149 & 464013686071 & -0.6251059 & \\
\hline & & & 07 & \\
\hline & & & & \\
\hline & & -0.41581592056993744019 & & 4591057369 \\
\hline & & & -1.2729882501 & 4591057369 \\
\hline & 20002296861 & 0.69159903931302930309 & -1.54651089221038991675 & 0.624 \\
\hline 14 & & & & \\
\hline & & & 3217 & 786 \\
\hline & & 79 & & 786 \\
\hline & & & 69 & \\
\hline & & & & \\
\hline $13 \omega$ & 9 & 56 & 12 & \\
\hline & & & & 8273235 \\
\hline & & & & \\
\hline & & & & \\
\hline & & & & -0.77603825525821386210 \\
\hline & -0.23 & 0.8 & & \\
\hline & & & & \\
\hline & & & & \\
\hline & & & -0.50097941898851340879 & 0.78841451122577084077 \\
\hline $16+\omega$ & -0.94732289744543974737 & -0.63159695361495073162 & 0.50097941898851340879 & -0.78841451122577084077 \\
\hline
\end{tabular}

Tabelle 3.2: Liste der ersten fünfundfünzig $c(p)$ für $R \in\left\{R_{1}^{+}, R_{1}^{-}, R_{2}^{-}, R_{14}^{-}\right\}$ 


\section{Kapitel 4}

\section{Fourierkoeffizienten metaplektischer Formen}

In Kapitel 3 wurden automorphe Formen nach dem folgenden Schema bestimmt: Zu einer Basis von Eigenfunktionen zu $\Delta$ auf $\mathbb{H}^{3}$ wurden Linearkombinationen gesucht, welche den Randbedingungen $f=\gamma f, \forall \gamma \in \Gamma$ genügen. Durch die Summation wurde Invarianz gegen $T$ und $R$ gewährleistet, so daß als einzige Bedingung die Invarianz gegen $S$ blieb, welche durch Kollokation in der Nachbarschaft von $\mathcal{F}_{3}$ und $S \mathcal{F}_{3}$ gelöst wurde. Eine alternative Sichtweise dieses Vorgehens ist, daß $f$ auf $\stackrel{\circ}{\mathcal{F}}_{3}$ als Linearkombination von Eigenfunktionen zu $\Delta$ vorgegeben ist, durch die Gleichung $f=\gamma f$ auf $\bigcup_{\gamma \in \Gamma} \gamma \stackrel{\circ}{\mathcal{F}}_{3}$ fortgesetzt und dann durch Kollokation die stetige Differenzierbarkeit auf dem Rand erreicht wird.

Ein wesentlicher Unterschied metaplektischer Formen zur Berechnung automorpher Formen ergibt sich aus der Tatsache, daß die metaplektische Gruppe mehrere Spitzen hat. Um das Schema des letzten Kapitels zu verallgemeinern, muß nicht nur eine Fourierentwicklung zur Spitze $\infty$, sondern auch eine weitere Entwicklung, etwa um $1+\omega$, betrachtet werden. Dies genügt, um $f$ zu beschreiben, da $f$ ohne Einschränkung als gerade oder ungerade angenommen werden kann. Dann soll durch Kollokation die stetige Differenzierbarkeit von $f$ auf dem Rand beider Konvergenzgebiete gelöst werden.

In diesem Kapitel wird in den ersten drei Abschnitten die allgemeine Fourierdarstellung beschrieben, wobei notwendige Umformungen im Mittelpunkt stehen. Der allgemeine Fourieransatz läßt sich zwar in eine einfache Formel fassen, doch er beinhaltet eine Zahl von Redundanzen, welche sich in numerischen Bestimmungen negativ auswirken. Um Resultate erhalten zu können, 
war es erforderlich, die Darstellung auf verschiedene Weise zu modifizieren. Der vierte Abschnitt beschreibt dann den Rand der Entwicklungsgebiete um $\infty$ und $\omega+1$ sowie die Ausführung der Kollokation auf dem Rand.

Die Berechnungen in diesem Kapitel bilden die Grundlage für die numerische Umsetzung. Da alle eingesetzten Programme den quadratischen Körper zur Diskriminante -3 in einer Basis mit einer sechsten Einheitswurzel darstellen, werden im Folgenden viele Gleichungen zur Basis $\omega_{6}=\omega+1$ angegeben.

\subsection{Erste Vereinfachung der Fourierdarstellung}

Die in Kapitel 3 für automorphe Formen vorgestellte Idee muß für metaplektische Formen also noch erweitert werden: Alle Fourierentwicklungen müssen invariant gegen alle metaplektischen Transformationen sein. Während die Darstellungen (2.23) und (2.24) noch für einen allgemeineren Fall Gültigkeit haben, sollen diese Gleichungen durch Ausnutzen von Invarianzen möglichst stark vereinfacht werden, um sie so der Numerik besser zugänglich zu machen.

Die Darstellungen (2.23) und (2.24) ergeben explizit für eine metaplektische Spitzenform $f$ in der Umgebung der Spitze $\infty$ eine Entwicklung der Form

$$
f(z, v)=f_{\infty}(z, v)=\sum_{n \in \mathcal{O}}^{\prime} c_{\infty}(n) v K_{i R}\left(\frac{2 \pi}{3 A}|n| v\right) e\left(\frac{\langle i n, z\rangle}{3 A}\right)
$$

und für $(z, v)$ nahe der Spitze $\omega_{6}=\omega+1$ durch Einführung lokaler Koordinaten $\left(z_{\omega_{6}}, v_{\omega_{6}}\right)$ in der Form

$$
f_{\omega_{6}}\left(z_{\omega_{6}}, v_{\omega_{6}}\right)=f(z, v)=\sum_{n \in \mathcal{O}}^{\prime} c_{\omega_{6}}(n) v_{\omega_{6}} K_{i R}\left(\frac{2 \pi}{9 A}|n| v_{\omega_{6}}\right) e\left(\frac{\left\langle i n, z_{\omega_{6}}\right\rangle}{9 A}\right)
$$

mit

$$
\left(z_{\omega_{6}}, v_{\omega_{6}}\right)=\sigma_{\omega_{6}}(z, v)=\left(\begin{array}{cc}
0 & -1 \\
1 & -\omega_{6}
\end{array}\right)(z, v)=\left(-\frac{\overline{z-\omega_{6}}}{\left|z-\omega_{6}\right|^{2}+v^{2}}, \frac{v}{\left|z-\omega_{6}\right|^{2}+v^{2}}\right)
$$

das heißt $f_{\omega_{6}}(w)=\sigma_{\omega_{6}}^{-1} f_{\infty}(w)$, und $f_{\infty}$ und $\sigma_{\omega_{6}} f_{\omega_{6}}$ sind lokal gültige Entwicklungen von $f$ in den Spitzen $\infty$ bzw. $\omega_{6}$.

Da die metaplektischen Formen in gerade und ungerade zerfallen (vgl. 2.12) und als nicht äquivalente Spitzen $\infty, \omega_{6}$ und $-\omega_{6}$ gewählt werden können, ist eine Betrachtung der Fourier- 
entwicklung nur in den Spitzen $\infty$ und $\omega_{6}$ nötig, da sich die Entwicklung in $-\omega_{6}$ durch

$$
f_{\omega_{6}}(w)=f\left(\sigma_{\omega_{6}}(w)\right)=\varepsilon_{f} f\left(-\sigma_{\omega_{6}}(w)\right)=\varepsilon_{f} f\left(\sigma_{-\omega_{6}}(-w)\right)=\varepsilon_{f} f_{-\omega_{6}}(-w)
$$

mit $\varepsilon_{f} \in\{ \pm 1\}$ aus der in $\omega_{6}$ ableitet.

Die Wahl von $\mathcal{O}$ als Gitter in (4.2) muß erläutert werden: Für $\gamma \in \tilde{\Gamma}$ gilt $f(\gamma w)=\chi(\gamma) f(w)$, also

$$
f_{\omega_{6}}(\gamma w)=f\left(\sigma_{\omega_{6}}^{-1} \gamma w\right)=f\left(\sigma_{\omega_{6}}^{-1} \gamma \sigma_{\omega_{6}}\left(\sigma_{\omega_{6}}^{-1} w\right)\right)=\chi\left(\sigma_{\omega_{6}}^{-1} \gamma \sigma_{\omega_{6}}\right) f_{\omega_{6}}(w)
$$

für $\sigma_{\omega_{6}}^{-1} \gamma \sigma_{\omega_{6}} \in \tilde{\Gamma}$. Es ist $\sigma_{\omega_{6}}^{-1}\left(\begin{array}{cc}1 & 3 n \\ 0 & 1\end{array}\right) \sigma_{\omega_{6}} \in \tilde{\Gamma}$ für alle $n \in \mathcal{O}$, also

$$
\chi\left(\sigma_{\omega_{6}}{ }^{-1}\left(\begin{array}{cc}
1 & 9 n \\
0 & 1
\end{array}\right) \sigma_{\omega_{6}}\right)=\left(\chi\left(\sigma_{\omega_{6}}^{-1}\left(\begin{array}{cc}
1 & 3 n \\
0 & 1
\end{array}\right) \sigma_{\omega_{6}}\right)\right)^{3}=1
$$

so daß sich, wie in (4.2) gewählt, $\frac{1}{9} \mathrm{Z}[\omega]$ als Gitter von $f_{\omega_{6}}$ ergibt.

Tatsächlich kann man noch mehr zur Fourierentwicklung sagen. Während eine Verschiebung um ein Vielfaches von 9, wie oben gezeigt, trivial in $\chi$ ist, ist eine lokale Verschiebung um 3 zwar ebenfalls metaplektisch, allerdings nicht mit trivialem Charakter. Man kann dies benutzen, um die Fourierentwicklung weiter zu spezifizieren. Es gilt:

$$
\sigma_{\omega_{6}}{ }^{-1}\left(\begin{array}{ll}
1 & 3 \\
0 & 1
\end{array}\right) \sigma_{\omega_{6}}=\left(\begin{array}{cc}
1-3 \omega_{6} & -3-3 \omega_{6} \\
-3 & 1+3 \omega_{6}
\end{array}\right),
$$

also

$$
\chi\left(\sigma_{\omega_{6}}{ }^{-1}\left(\begin{array}{ll}
1 & 3 \\
0 & 1
\end{array}\right) \sigma_{\omega_{6}}\right)=\left(-3 / 1-3 \omega_{6}\right)_{3}=\omega
$$

und genauso

$$
\sigma_{\omega_{6}}^{-1}\left(\begin{array}{cc}
1 & 3 \omega_{6} \\
0 & 1
\end{array}\right) \sigma_{\omega_{6}}=\left(\begin{array}{cc}
4+3 \omega_{6} & 3 \\
-3 \omega_{6} & -2-3 \omega_{6}
\end{array}\right)
$$

also

$$
\chi\left(\sigma_{\omega_{6}}{ }^{-1}\left(\begin{array}{cc}
1 & 3 \omega_{6} \\
0 & 1
\end{array}\right) \sigma_{\omega_{6}}\right)=\left(-3 \omega_{6} / 4+3 \omega_{6}\right)_{3}=\omega .
$$

Eingesetzt in die Fourierentwicklung erhält man

$$
\begin{aligned}
\sum_{n \in \mathcal{O}}^{\prime} c_{\omega_{6}}(n) v K_{i R}\left(\frac{2 \pi}{9 A}|n| v\right) e\left(\frac{\langle i n, z\rangle}{9 A}\right) & =\omega_{6}^{-1} \sum_{n \in \mathcal{O}}^{\prime} c_{\omega_{6}}(n) v K_{i R}\left(\frac{2 \pi}{9 A}|n| v\right) e\left(\frac{\left\langle i n, z+3 \omega_{6}\right\rangle}{9 A}\right) \\
& =e^{\frac{-4 \pi i}{3}} \sum_{n \in \mathcal{O}}^{\prime} c_{\omega_{6}}(n) v K_{i R}\left(\frac{2 \pi}{9 A}|n| v\right) e\left(\frac{\langle i n, z\rangle}{9 A}\right) e\left(\frac{\left\langle i n, 3 \omega_{6}\right\rangle}{9 A}\right)
\end{aligned}
$$


gleichbedeutend mit

$$
e\left(-\frac{2}{3}+\frac{\left\langle i n, 3 \omega_{6}\right\rangle}{9 A}\right) \neq 1 \Rightarrow c_{\omega_{6}}(n)=0
$$

und damit

$$
-2 A+\left\langle i n, \omega_{6}\right\rangle \not \equiv 0 \quad(\bmod 3 A) \Rightarrow c_{\omega_{6}}(n)=0
$$

und genauso erhält man für

$$
\sum_{n \in \mathcal{O}}^{\prime} c_{\omega_{6}}(n) v K_{i R}\left(\frac{2 \pi}{9 A}|n| v\right) e\left(\frac{\langle i n, z\rangle}{9 A}\right)=\omega_{6}^{-1} \sum_{n \in \mathcal{O}}^{\prime} c_{\omega_{6}}(n) v K_{i R}\left(\frac{2 \pi}{9 A}|n| v\right) e\left(\frac{\langle i n, z+3\rangle}{9 A}\right)
$$

die Identität

$$
-2 A+\langle i n, 1\rangle \not \equiv 0 \quad(\bmod 3 A) \Rightarrow c_{\omega_{6}}(n)=0 .
$$

Mit $n=n_{1}+n_{2} \omega_{6}$ und $A=\frac{\sqrt{3}}{2}$ gilt

$$
\left\langle i n, \omega_{6}\right\rangle=\operatorname{Re} i n \overline{\omega_{6}}=-\frac{\sqrt{3}}{2} n_{1} \quad \text { und } \quad\langle i n, 1\rangle=\operatorname{Re} i n=-\frac{\sqrt{3}}{2} n_{2} .
$$

Also verschwinden an der Spitze $\omega_{6}$ alle Fourierkoeffizienten mit Ausnahme derer, für die gilt $-2-n_{1} \equiv 0 \quad(\bmod 3)$ und $-2-n_{2} \equiv 0 \quad(\bmod 3)$, zusammen:

$$
n \equiv 1+\omega_{6} \quad(\bmod 3)
$$

Im Folgenden sei entsprechend

$$
n_{\omega_{6}}=1+\omega_{6}+3 n
$$

Daraus ergibt sich eine Fourierentwicklung der Form

$$
f_{\omega_{6}}(z, v)=\sum_{n \in \mathcal{O}} c_{\omega_{6}}\left(n_{\omega_{6}}\right) v K_{i R}\left(\frac{2 \pi}{9 A}\left|n_{\omega_{6}}\right| v\right) e\left(\frac{\left\langle i n_{\omega_{6}}, z\right\rangle}{9 A}\right) .
$$

\subsection{Zweite Vereinfachung der Fourierdarstellung}

Darüber hinaus ist noch eine weitere Vereinfachung möglich, wenn man ausnutzt, daß die lokale 2 $\pi$ /3-Drehung: $R_{d}=\left(\begin{array}{cc}\omega_{6}^{2} & -1 \\ 0 & -\omega_{6}\end{array}\right)$ global metaplektisch ist:

$$
\sigma_{\omega_{6}}^{-1}\left(\begin{array}{cc}
1+\omega_{6} & -1 \\
0 & -\omega_{6}
\end{array}\right) \sigma_{\omega_{6}}=\left(\begin{array}{ll}
0 & -1 \\
1 & -1
\end{array}\right) \in \tilde{\Gamma}
$$


vergleiche auch Abbildung 2.5(c) und deren Beschreibung. Mit Gleichung (4.4) und $\chi\left(\left(\begin{array}{ll}0 & -1 \\ 1 & -1\end{array}\right)\right)=$ 1 haben wir $f_{\omega_{6}}(w)=f_{\omega_{6}}\left(\left(\begin{array}{cc}\omega_{6}^{2} & -1 \\ 0 & -\omega_{6}\end{array}\right) w\right)$, also

$$
\begin{aligned}
f_{\omega_{6}}(w) & =\sum_{n \in \mathcal{O}} c_{\omega_{6}}\left(n_{\omega_{6}}\right) v K_{i R}\left(\frac{2 \pi}{9 A}\left|n_{\omega_{6}}\right| v\right) e\left(\frac{\left\langle i n_{\omega_{6}},-\omega_{6} z-\omega_{6}{ }^{2}\right\rangle}{9 A}\right) \\
& =\sum_{n \in \mathcal{O}} c_{\omega_{6}}\left(n_{\omega_{6}}\right) v K_{i R}\left(\frac{2 \pi}{9 A}\left|n_{\omega_{6}}\right| v\right) e\left(\frac{\left\langle i n_{\omega_{6}},-\omega_{6}{ }^{2}\right\rangle}{9 A}\right) e\left(\frac{\left\langle-i \overline{\omega_{6}} n_{\omega_{6}}, z\right\rangle}{9 A}\right) \\
& =\sum_{n \in \mathcal{O}} c_{\omega_{6}}\left(-\omega_{6} n_{\omega_{6}}\right) v K_{i R}\left(\frac{2 \pi}{9 A}\left|n_{\omega_{6}}\right| v\right) e\left(\frac{\left\langle-i \omega_{6} n_{\omega_{6}},-\omega_{6}{ }^{2}\right\rangle}{9 A}\right) e\left(\frac{\left\langle i n_{\omega_{6}}, z\right\rangle}{9 A}\right) \\
& =\sum_{n \in \mathcal{O}} c_{\omega_{6}}\left(-\omega_{6} n_{\omega_{6}}\right) v K_{i R}\left(\frac{2 \pi}{9 A}\left|n_{\omega_{6}}\right| v\right) e\left(\frac{\left\langle i n_{\omega_{6}}, \omega_{6}\right\rangle}{9 A}\right) e\left(\frac{\left\langle i n_{\omega_{6}}, z\right\rangle}{9 A}\right)
\end{aligned}
$$

Vergleich von $\left(4.2^{\prime}\right)$ und (4.8) liefert

$$
c_{\omega_{6}}\left(-\omega_{6} n_{\omega_{6}}\right)=c_{\omega_{6}}\left(n_{\omega_{6}}\right) e\left(-\frac{\left\langle i n_{\omega_{6}}, \omega_{6}\right\rangle}{9 A}\right),
$$

wobei $-\omega_{6} n_{\omega_{6}}=\left(1+\omega_{6}+3\left(-\omega_{6} n-\omega_{6}\right)\right)$ und so $-\omega_{6} n_{\omega_{6}}=n_{\omega_{6}}^{\prime}$ mit $n^{\prime}=-\omega_{6} n-\omega_{6}$ gilt. Das Quadrat dieser Abbildung, $\left(\begin{array}{cc}\omega_{6} & -1 \\ 0 & \omega_{6}^{2}\end{array}\right):(z, v) \mapsto\left(\omega_{6}^{2} z-\omega_{6}, v\right)$, liefert analog

$$
c_{\omega_{6}}\left(\omega_{6}^{2} n_{\omega_{6}}\right)=c_{\omega_{6}}\left(n_{\omega_{6}}\right) e\left(-\frac{\left\langle i \omega_{6}^{2} n_{\omega_{6}},-\omega_{6}\right\rangle}{9 A}\right)=c_{\omega_{6}}\left(n_{\omega_{6}}\right) e\left(\frac{\left\langle i n_{\omega_{6}}, \omega_{6}{ }^{2}\right\rangle}{9 A}\right)
$$

wiederum mit $\omega_{6}^{2} n_{\omega_{6}}=\left(1+\omega_{6}+3\left(1+n\left(\omega_{6}-1\right)\right)\right)$ also $-\omega_{6} n_{\omega_{6}}=n_{\omega_{6}}^{\prime \prime}$ mit $n^{\prime \prime}=1+n\left(\omega_{6}-1\right)$. Aus $\left(4.2^{\prime}\right)$, (4.9) und (4.10) ergibt sich die Fourierdarstellung

$$
f_{\omega_{6}}(z, v)=\sum_{n \in \mathcal{O} /\left\{1, \omega, \omega^{2}\right\}} c_{\omega_{6}}\left(n_{\omega_{6}}\right) v K_{i R}\left(\frac{2 \pi}{9 A}\left|n_{\omega_{6}}\right| v\right) \operatorname{cs}_{n}(z)
$$

mit

$$
\operatorname{cs}_{n}(z)=e\left(\frac{\left\langle i n_{\omega_{6}}, z\right\rangle}{9 A}\right)+e\left(\frac{\left\langle i \omega_{6} n_{\omega_{6}}, z\right\rangle}{9 A}\right) e\left(\frac{\left\langle i n_{\omega_{6}},-\omega_{6}\right\rangle}{9 A}\right)+e\left(\frac{\left\langle i \omega_{6}{ }^{2} n_{\omega_{6}}, z\right\rangle}{9 A}\right) e\left(\frac{\left\langle i n_{\omega_{6}}, \omega_{6}{ }^{2}\right\rangle}{9 A}\right)
$$

und $n_{\omega_{6}}=1+\omega_{6}+3 n$ wie in (4.7).

Auch die Entwicklung (4.1) um $\infty$ läßt sich vereinfachen (vgl. Abbildung 2.5(a)): Hier ist $T=\left(\begin{array}{ll}1 & 1 \\ 0 & 1\end{array}\right)$ metaplektisch mit $\chi(T)=1$, daher wirkt $T:(z, r) \mapsto(z+1, r)$ trivial in der Fourierreihe und man kann $\mathcal{O}^{\infty}=\left\{n_{1}+n_{2} \omega \mid n_{2} \equiv 0 \quad(\bmod 3)\right\}$ als Gitter wählen und erhält

$$
f_{\infty}(z, v)=\sum_{n \in \mathcal{O}^{\infty}}^{\prime} c_{\infty}(n) v K_{i R}\left(\frac{2 \pi}{3 A}|n| v\right) e\left(\frac{\langle i n, z\rangle}{3 A}\right) .
$$


Dies kann man noch einmal vereinfachen, indem man ausnutzt, daß $f$ entweder als gerade oder als ungerade vorauszusetzen ist. In beiden Fällen kann man die beiden $\mathrm{zu}+n$ und $-n$ gehörenden Exponentialterme zusammenfassen und erhält

$$
f_{\infty}(z, v)=\sum_{n \in \mathcal{O}^{\infty} /\{ \pm 1\}}^{\prime} c_{\infty}(n) v K_{i R}\left(\frac{2 \pi}{3 A}|n| v\right) \varphi_{n}(z)
$$

wobei $\left.\varphi_{n}(z)=\begin{array}{c}\cos \\ \sin \end{array}\right\}\left(\frac{\langle i n, z\rangle}{3 A}\right)$, je nachdem ob $f$ gerade oder ungerade.

\subsection{Dritte Vereinfachung der Fourierdarstellung}

Bei der Berechnung der Eigenfunktionen hat sich herausgestellt, daß es für eine stabile Numerik nötig ist, diese Darstellung noch weiter zu simplifizieren. Aus der Funktionalgleichung mit $\gamma=$ $\left(\begin{array}{ll}a & b \\ c & d\end{array}\right) \in \tilde{\Gamma}$ folgt mit der Definition des Kubotacharakters $\chi$ aus $\gamma f(z, v)=\chi(\gamma) f(z, v)$ bei komplexer Konjugation direkt, daß

$$
\bar{\gamma} f(\bar{z}, v)=\chi(\gamma) f(\bar{z}, v)
$$

gilt. Daher ist mit $f$ auch $f^{*}:(z, v) \mapsto \bar{f}(\bar{z}, v)$ metaplektisch. Da für nicht verschwindendes $f$ nicht sowohl $f+f^{*}$ als auch $f-f^{*}$ identisch verschwinden, kann man von einer metaplektischen Form $f$ die Eigenschaft

$$
f(z, v)=\bar{f}(\bar{z}, v)
$$

verlangen, denn sowohl $f+f^{*}$ als auch $i\left(f-f^{*}\right)$ erfüllen (4.12). Dies überträgt sich direkt auch im mehrdimensionalen Eigenraum. Damit ergibt 4.12 eine Einschränkung für $f$, welche sich direkt in die Entwicklung um die Spitze $\infty$ überträgt. Für die Entwicklung um die Spitze $\omega_{6}$ sei wieder $\left(z_{\omega_{6}}, v_{\omega_{6}}\right)=\sigma_{\omega_{6}}(z, v)$. Dann gilt

$$
f_{\omega_{6}}\left(z_{\omega_{6}}, v_{\omega_{6}}\right)=f(z, v)=f(z-1, v)=\bar{f}(\bar{z}-1, v)=\varepsilon_{f} f(1-\bar{z})=\varepsilon_{f} \bar{f}_{\omega_{6}}\left(\sigma_{\omega_{6}}(1-\bar{z}, v)\right)
$$

und mit $\omega_{6}=1-\overline{\omega_{6}}$ ist

$$
\sigma_{\omega_{6}}(1-\bar{z}, v)=\left(-\frac{\overline{1-\bar{z}-\omega_{6}}}{\left|1-\bar{z}-\omega_{6}\right|^{2}+v^{2}}, \frac{v}{\left|1-\bar{z}-\omega_{6}\right|^{2}+v^{2}}\right)=\left(\frac{z-\omega_{6}}{\left|z-\omega_{6}\right|^{2}+v^{2}}, \frac{v}{\left|z-\omega_{6}\right|^{2}+v^{2}}\right) .
$$

Da die rechte Seite gerade $\left(-\overline{z_{\omega_{6}}}, v_{\omega_{6}}\right)$ ist, gilt also

$$
f_{\omega_{6}}\left(z_{\omega_{6}}, v_{\omega_{6}}\right)=\varepsilon_{f} \bar{f}_{\omega_{6}}\left(-\overline{z_{\omega_{6}}}, v_{\omega_{6}}\right)
$$


Diese Einschränkungen lassen sich wie folgt in die Entwicklungen einsetzen: Vereinfachend geschrieben war $\left(4.1^{\prime \prime}\right)$ von der Form

$$
f(w)=\sum_{n \in \mathcal{O}^{(\infty)}} A_{n}(v) \varphi_{n}(z)
$$

mit

$$
\mathcal{O}^{(\infty)}=\left\{n_{1}+3 n_{2} \omega_{6} \mid n_{i} \in \mathrm{Z}, n_{1}<0,0 \leq 3 n_{2} \leq-2 n_{1}\right\}
$$

Die $\varphi_{n}$ sind reell, und es gilt $\varphi_{n}(\bar{z})=\varphi_{-\bar{n}}(z)$. Führt man nun

$$
\varphi_{n}^{+}(z)=\varphi_{n}(z)+\varphi_{-\bar{n}}(z) \text { und } \varphi_{n}^{-}(z)=\varphi_{n}(z)-\varphi_{-\bar{n}}(z)
$$

ein, so wird

$$
f(w)=\sum_{n \in \mathcal{O}(\infty)} A_{n}^{+}(v) \varphi_{n}^{+}(z)+i A_{n}^{-}(v) \varphi_{n}^{-}(z) .
$$

Hierbei sind die $A_{n}^{+}(v)$ und $A_{n}^{-}(v)$ reell und die Grenzen gerade bei $n= \pm \bar{n}$. Auf dem Rand ist bei

$$
\begin{aligned}
n=+\bar{n}: & \varphi_{n}^{+}(z)=\varphi_{n}(z)+\varphi_{-n}(z)=\left(1+\varepsilon_{f}\right) \varphi_{n}(z) \\
& \varphi_{n}^{-}(z)=\left(1-\varepsilon_{f}\right) \varphi_{n}(z) \\
n=-\bar{n}: & \varphi_{n}^{+}(z)=2 \varphi_{n}(z) \\
& \varphi_{n}^{-}(z)=0 .
\end{aligned}
$$

Bei der Spitze $\omega_{6}$ ist die Reihenentwicklung nach $\left(4.2^{\prime \prime}\right)$ von der Form

$$
f_{\omega_{6}}(w)=\sum_{n \in \mathcal{O}^{\left(\omega_{6}\right)}} B_{n}(v) \operatorname{cs}_{n}(z)
$$

mit

$$
\mathcal{O}^{\left(\omega_{6}\right)}=\left\{3\left(n_{1}+n_{2} \omega_{6}\right)-2\left(1+\omega_{6}\right) \mid n_{i} \in \mathrm{Z}, 0<n_{1}, 2-2 n_{1} \leq n_{2} \leq 1-n_{1} / 2\right\} .
$$

Für die $\operatorname{cs}_{n}(z)$ aus Gleichung (4.11) gilt

$$
\operatorname{cs}_{n}(\bar{z})=\operatorname{cs}_{-\bar{n}}(z), \overline{\operatorname{cs}_{n}(z)}=\operatorname{cs}_{n}(-z), \overline{c s_{n}(-\bar{z})}=\operatorname{cs}_{-\bar{n}}(z)
$$

und mit der analogen Definition

$$
\operatorname{cs}_{n}^{ \pm}(z)=\operatorname{cs}_{n}(z) \pm \operatorname{cs}_{-\bar{n}}(z)
$$


gilt

$$
f_{\omega_{6}}(w)=\sum_{n \in \mathcal{O}^{\left(\omega_{6}\right)}} B_{n}^{+}(v) \operatorname{cs}_{n}^{+}(z)+\sum_{n \in \mathcal{O}^{\left(\omega_{6}\right)}} B_{n}^{-}(v) \operatorname{cs}_{n}^{-}(z) .
$$

Wenn man hier nun (4.13) einsetzt, erhält man

$$
0=\bar{f}_{\omega_{6}}(z, v)-\varepsilon_{f} f_{\omega_{6}}(-\bar{z}, v)=\sum\left(B_{n}^{+}(v)-\varepsilon_{f} \bar{B}_{n}^{+}(v)\right) \operatorname{cs}_{n}^{+}(z)+\sum\left(B_{n}^{-}(v)-\varepsilon_{f} \bar{B}_{n}^{-}(v)\right) \operatorname{cs}_{n}^{-}(z)
$$

Da die $\varepsilon_{f} \in\{ \pm 1\}$ anzeigen, ob es sich um eine gerade oder eine ungerade Funktion handelt, folgt, daß die $B_{n}^{+}(v)$ für gerade Funktionen reell sind und für ungerade Funktionen imaginär, während es bei den $B_{n}^{-}(v)$ gerade umgekehrt ist. Auf dem gesamten Rand ist $\mathrm{cs}_{n}^{-}=0$ und $\mathrm{cs}_{n}^{+}=2 \mathrm{cs}_{n}$, so daß die $B_{n}^{-}(v)$ für $2 n_{1}+n_{2}=2$ und $n_{1}+2 n_{2}=2$ verschwinden.

\subsection{Ausführung der Kollokation}

Die Erkenntnisse der ersten drei Abschnitte sollen nun genutzt werden, um die Kollokation durchzuführen. In Kapitel 3 wurde eine Kollokation für automorphe $f$ durchgeführt. Diese nutzte die Gleichung $f(w)=S f(w)$ auf der Grenze zwischen $\mathcal{F}_{3}$ und $R S \mathcal{F}_{3}$ an $m$ Punkten $w_{i}$ aus, um für eine Approximation für $f$ von der Form $\sum_{j=1}^{n} c_{j} \varphi_{j}(w)$ dann $m$ lineare Gleichungen mit $n$ Unbekannten zu haben. Diese Grenze stellt ein drittel Sechseck auf der Einheitskugel dar (vgl. Abbildung 2.2). Diese Grundidee kann man recht analog auch für metaplektisches $f$ in lokalen Koordinaten durchführen.

Um ein gut konditioniertes Gleichungssystem zu erhalten, ist es allerdings von entscheidender Bedeutung, die Kollokationspunkte so zu wählen, daß sie den Kollokationsbereich möglichst gleichmäßig überdecken. Hierzu ist eine genauere Analyse der Kollokationsgleichungen erforderlich, die hier ausgeführt werden soll.

Durch geeignete Wahl der Transformationen lassen sich als Kollokationsflächen immer Teile von Einheitskugeln in lokalen Koordinaten wählen. Zur metaplektischen Funktion $f_{w_{s}}$ zur Spitze $w_{s}$ soll also eine Kollokationsfunktion $\varphi_{w_{s}}(\eta+m)=f_{w_{s}}\left(\eta+m, \sqrt{1-|\eta|^{2}}\right)$ zu einem Mittelpunkt $m$ gewählt werden.

Da $\tilde{\Gamma}$ aus 27 Kopien von $\Gamma$ besteht, gibt es neun sechseckige Grundseiten, welche einer Kollokation zugänglich sind. Die Betrachtungen in Kapitel 2, speziell zu Abbildung 2.7 ergeben, 
daß von diesen neun Bereichen sechs doppelt und drei einfach gezählt werden, so daß insgesamt sechs Grundseiten zur Verfügung stehen. Der Rest dieses Abschnittes soll diese Fläche auf zwei halbe Sechsecke und zwei viertel Sechsecke bei gleichzeitiger Vereinfachung der Gleichungen reduzieren.

Bei den angesprochenen sechs Flächen, mit denen wir beginnen, handelt es sich um die in Tabelle 4.1 angegebenen Nachbarschaften. In dieser Tabelle fixieren die angegebenen Abbildun-

(Nachbarschaft) $\quad \gamma \in \tilde{\Gamma} \quad: \quad$ Indizes der benachbarten Bereiche

$$
\begin{aligned}
& \left(\mathbf{N}_{1}\right) \quad S \quad: \quad 1,2,3 \rightleftharpoons 1,2,3 \\
& \left(\mathbf{N}_{2}\right) \quad \text { id } \quad: \quad 4,5,6 \rightleftharpoons 19,20,21 \\
& \left(\mathbf{N}_{3}\right) \quad L^{3} \quad: \quad 16,17,18 \rightleftharpoons 25,26,27 \\
& \left(\mathbf{N}_{4}\right) \quad R_{1} T^{-1} \quad: \quad 22,23,24 \rightleftharpoons 22,23,24 ; \quad R_{1}=\left(\begin{array}{cc}
1 & -1 \\
1 & 0
\end{array}\right) \\
& \left(\mathbf{N}_{5}\right) \quad \text { id } \quad: \quad 7,8,9 \rightleftharpoons 10,11,12 \\
& \left(\mathbf{N}_{6}\right) \quad R_{2} T: 13,14,15 \rightleftharpoons 13,14,15 ; \quad R_{2}=\left(\begin{array}{cc}
1 & 1 \\
-1 & 0
\end{array}\right) \text {, }
\end{aligned}
$$

Tabelle 4.1: Nichttriviale Nachbarschaften

gen den gemeinsamen Rand der beiden Vereinigungen dreier Gebiete, welche durch deren Indizes angegeben sind. Zuerst sei angemerkt, daß sich die $f$ mit $f(z, v)=\varepsilon_{f} f(-z, v)$ in gerade und ungerade $f$ aufteilen, so daß sich die Nachbarschaftsbedingungen für $\mathbf{N}_{5}$ und $\mathbf{N}_{6}$ aus den Bedingungen von $\mathbf{N}_{2}$ bzw. $\mathbf{N}_{4}$ herleiten lassen. Damit bleiben für $\mathbf{N}_{1}$ eine Gleichung für $\varphi_{\infty}$, für $\mathbf{N}_{2}$ eine Beziehung zwischen $\varphi_{\infty}$ und $\varphi_{\omega_{6}}$ und für $\mathbf{N}_{3}$ und $\mathbf{N}_{4}$ Gleichungen für $\varphi_{\omega_{6}}$.

Bei $\mathbf{N}_{1}$ übertragen sich zuerst einmal die Verhältnisse aus Kapitel 2. Die einfachste Form der Kollokation ist

$$
\varphi_{\infty}(\eta+0)=\varphi_{\infty}(-\bar{\eta}+0)
$$

Unter $z \mapsto-z$ wird dies durch $\varphi_{\infty}(\eta+0)=f_{\infty}\left(\eta+0, \sqrt{1-|\eta|^{2}}\right) \mathrm{zu}$

$$
\varphi_{\infty}(-\eta+0)=\varphi_{\infty}(\bar{\eta}+0)
$$

und unter $z \mapsto \bar{z} \mathrm{zu}$

$$
\varphi_{\infty}(\bar{\eta}+0)=\varphi_{\infty}(-\eta+0)
$$


Man erkennt, daß jede Reihe (4.1'I') für $-\eta_{0}$ und $\pm \bar{\eta}_{0}$ die Gleichung (4.15) erfüllt, wenn sie sie für $\eta_{0}$ erfüllt, so daß wir lediglich ein viertel Sechseck als Kollokationsfläche erhalten. Zusammen mit (4.12) liefert (4.15) dann

$$
\varepsilon_{f} \overline{\varphi_{\infty}(-\bar{\eta}+0)}=\varphi_{\infty}(\eta+0)=\varphi_{\infty}(-\bar{\eta}+0)
$$

Daran sieht man, daß für gerade Funktionen die Realteile und für ungerade die Imaginärteile implizit übereinstimmen. Anstelle einer komplexen Gleichung erhält man also die reelle Gleichung

$$
\left\{\begin{array}{l}
\varepsilon_{f}=1: \quad \operatorname{Im} \\
\varepsilon_{f}=-1: \operatorname{Re}
\end{array}\right\} \varphi_{\infty}(\eta+0)=0
$$

das heißt, die betrachteten metaplektischen Funktionen sind auf der Einheitskugel reell bzw. imaginär.

Für $\mathbf{N}_{2}$ ist die Kollokation vollständig innerhalb des gewählten Fundamentalbereiches. Direktes Einsetzen der lokalen Koordinaten liefert die Beziehung

$$
\varphi_{\omega_{6}}(-\bar{\eta}+0)=\varphi_{\infty}(\eta+(\omega+1))
$$

mit $\eta=z-(\omega+1)$. Wie man sieht, ist (4.17) nicht invariant unter $\eta \mapsto-\bar{\eta}$, aber mit

$$
\bar{f}_{\omega_{6}}(\bar{z}, v)=f\left(\bar{\sigma}_{\omega_{6}}(z, v)=f_{1-\omega_{6}}(z, v)=f_{-\omega_{6}}(z, v)=\varepsilon_{f} f_{\omega_{6}}(-z, v)\right.
$$

folgt aus der Gültigkeit von (4.17) für $\eta$ dieselbe für $-\bar{\eta}$ :

$$
\varphi_{\omega_{6}}(-\bar{\eta}+0)=\varphi_{\infty}(\eta+(\omega+1))
$$

so daß man sich bei der Kollokation $\mathbf{N}_{2}$ auf ein halbes Sechseck, etwa mit $\operatorname{Re} \eta \geq 0$, beschränken kann.

Bei $\mathbf{N}_{4}$ stellt sich die Situation etwas anders dar. Die Grundfläche von 22, 23, 24 gehört zur Spitze $\omega+1$ und liegt $\omega_{6}$-lokal auf der Kugel um -1 (vgl. Abbildung 2.5(c)). Wie man leicht nachrechnen kann, ist die globale Nachbarschaftsbeziehung durch die Transformation $R_{1} T^{-1}=$ $T R_{1}^{-1}=\left(\begin{array}{ll}1 & -2 \\ 1 & -1\end{array}\right)$ gegeben (vgl. Abbildung 2.3). Für die lokale Reihe ergibt sich damit

$$
\begin{aligned}
f_{\omega_{6}}(z, v) & =\sigma_{\omega_{6}}\left(\begin{array}{l}
1 \\
1-2
\end{array}\right) \sigma_{\omega_{6}}^{-1} f_{\omega_{6}}(z, v) \\
& =\chi\left(\left(\begin{array}{l}
1 \\
1-2 \\
1-1
\end{array}\right)\right) f_{\omega_{6}}\left(\frac{(\omega z-1) \overline{(-\omega z-\omega)}-\omega \bar{\omega} v^{2}}{|(-\omega z-\omega)|^{2}+|-\omega|^{2} v^{2}}, \frac{v}{|z+1|^{2}+v^{2}}\right) .
\end{aligned}
$$


Für $z$ lokal auf der Kugel um -1 haben wir mit $|z+1|^{2}+v^{2}=1$

$$
\begin{aligned}
\varphi_{\omega_{6}}((z+1)-1) & =f_{\omega_{6}}\left(-\omega(\bar{z}+1)-|z+1|^{2}-v^{2}, v\right) \\
& =\varphi_{\omega_{6}}(-\omega(\bar{z}+1)-1),
\end{aligned}
$$

was sich mit $\omega_{6}(z+1)=\eta \mathrm{zu}$

$$
\varphi_{\omega_{6}}\left(\omega_{6} \eta-1\right)=\varphi_{\omega_{6}}\left(-\omega_{6} \bar{\eta}-1\right)
$$

symmetrisieren läßt. Gleichung (4.19) ist gegen $\eta \mapsto-\bar{\eta}$ invariant, so daß auch hier die Kollokation auf ein halbes Sechseck beschränkt wurde. Allerdings läßt sich das mit (4.12) noch vereinfachen:

$$
\varphi_{\omega_{6}}\left(\omega_{6} \eta-1\right)=\varepsilon_{f} \overline{\varphi_{\omega_{6}}\left(-\overline{\omega_{6} \eta}+1\right)}=\varepsilon_{f} \overline{\varphi_{\omega_{6}}\left(\omega_{6} \bar{\eta}-1\right)}
$$

so daß mit (4.19)

$$
\varphi_{\omega_{6}}\left(\omega_{6} \eta-1\right)=\varepsilon_{f} \overline{\varphi_{\omega_{6}}\left(\omega_{6} \eta-1\right)}
$$

und so

$$
\left\{\begin{array}{l}
\varepsilon_{f}=1: \quad \operatorname{Im} \\
\varepsilon_{f}=-1: \operatorname{Re}
\end{array}\right\} \varphi_{\omega_{6}}\left(\omega_{6} \eta-1\right)=0
$$

folgt. Also ist auch für $\mathbf{N}_{4}$ eine reelle Gleichung in einem halben Sechseck zu lösen.

Schließlich zur Beziehung $\mathbf{N}_{3}$ : Hier geht es um die Nachbarschaft der Spitze $\omega_{6}$ zur Spitze $-\omega_{6}$. Wie Abbildung 2.3 zeigt, ist die Grundfläche der Bereiche 25, 26 und 27 durch $z \mapsto z-3 \omega_{6}$ mit der Grundfläche von 16, 17 und 18 verbunden. Abbildung 2.5 (b), (c) zeigt, daß 25, 26 und $27 \omega_{6}$-lokal auf einer Kugel um $\omega$ und 16, 17 und $18-\omega_{6}$-lokal auf einer Kugel um $-\omega$ stehen. In Formeln ergibt sich dies durch

$$
\begin{aligned}
f_{\omega_{6}}(z, v) & =\sigma_{-\omega_{6}}\left(\begin{array}{cc}
1 & -3 \omega_{6} \\
0 & 1
\end{array}\right) \sigma_{\omega_{6}}^{-1} f_{\omega_{6}}(z, v)=\left(\begin{array}{rr}
1 & 0 \\
\omega+1 & 1
\end{array}\right) f_{\omega_{6}}(z, v) \\
& =\chi\left(\left(\begin{array}{cc}
1 & -3 \omega_{6} \\
0 & 1
\end{array}\right)\right) f_{-\omega_{6}}\left(\frac{z \overline{((\omega+1) z+1)}+\overline{(\omega+1)} v^{2}}{|(\omega+1) z+1|^{2}+|\omega+1|^{2} v^{2}}, \frac{v}{|(\omega+1) z+1|^{2}+v^{2}}\right),
\end{aligned}
$$

weiter auf der Einheitskugel $|z-\omega|^{2}+v^{2}=1$

$$
\begin{aligned}
\varphi_{\omega_{6}}((z-\omega)+\omega) & =f_{-\omega_{6}}\left(-\omega+\frac{\omega(-\omega \bar{z}+1)}{|z-\omega|^{2}+v^{2}}, v\right) \\
& =f_{-\omega 6}((\omega+1) \bar{z}, v) \\
& =\varphi_{-\omega 6}((\omega+1)(\overline{z-\omega})-\omega)
\end{aligned}
$$


und mit $(z-\omega)=-i \omega \eta$

$$
\varphi_{\omega_{6}}(-i \omega \eta+\omega)=\varphi_{-\omega 6}(i \omega \bar{\eta}-\omega)=\varphi_{\omega 6}(-i \omega \bar{\eta}+\omega)
$$

und damit ist (4.20) sowohl bei $\eta \mapsto-\bar{\eta}$ als auch bei $\eta \mapsto-\eta$ invariant, so daß für $\mathbf{N}_{3}$ eine Beschränkung auf $\operatorname{Re} \eta>0, \operatorname{Im} \eta>0$ möglich ist.

Tatsächlich war es erforderlich, um die stetige Differenzierbarkeit bis auf den Rand zu erhalten, neben einer Kollokation der Funktion noch eine Kollokation der Normalableitung zu verlangen. Ein Versuch analog zu Kapitel 3, nur die Funktion in einem Bereich in der Nähe der Grenzfläche zu kollokieren, erwies sich als numerisch völlig instabil.

Sei $\eta=\eta_{r}+i \eta_{i}$ und $\nu=\sqrt{1-|\eta|^{2}}$, so gilt auf der Kugel entsprechend $1=\eta_{i}^{2}+\eta_{r}^{2}+\nu^{2}$. Die kontravarianten Komponenten eines Normaleneinheitsvektors sind in der hyperbolischen Metrik dann durch

$$
\mathbf{n}=\left(\frac{\eta_{r}}{\nu}, \frac{\eta_{i}}{\nu}, 1\right) \text { gegeben, da } g_{i k}=\frac{1}{\nu^{2}} \delta_{i k}, g^{i k}=\nu^{2} \delta_{i k}
$$

Damit ist die Normalenableitung

$$
\frac{\partial \varphi}{\partial \mathbf{n}}=\nu\left(\eta_{r} \frac{\partial \varphi}{\partial \eta_{r}}+\eta_{i} \frac{\partial \varphi}{\partial \eta_{i}}+\nu \frac{\partial \varphi}{\partial \nu}\right)
$$

Für den Spezialfall, daß $\varphi$ nur eine Funktion von $\pi_{a}(\eta)=\langle a, \eta\rangle=\eta_{r} \operatorname{Re} a+\eta_{i} \operatorname{Im} a$ ist, folgt

$$
\frac{\partial \varphi}{\partial \mathbf{n}}=\nu\left(\pi_{a} \frac{\partial \varphi}{\partial \pi_{a}}+\nu \frac{\partial \varphi}{\partial \nu}\right)
$$

Mit diesem Ansatz lassen sich die Normalenableitungen ganz analog zu den Funktionen kollokieren, was hier nicht mehr im einzelnen ausgeführt werden soll. $\mathrm{Zu}$ beachten ist dabei, daß die Normalableitung jeweils in Richtung der inneren Normalen ausgeführt wird, so daß sich die Ableitungen auf den Nachbarschaften jeweils um das Vorzeichen unterscheiden. 


\title{
Kapitel 5
}

\section{Bestimmung der metaplektischen Formen}

\begin{abstract}
Als eine der interessantesten Fragen in diesem Zusammenhang stellt sich die nach der Verbindung zwischen den automorphen und den dazu gehörenden metaplektischen Formen. Wie in [11] dargestellt, gibt es eine kubische Shimura Korrespondenz zwischen der Menge der in Kapitel 3 beschriebenen automorphen Formen und den in Kapitel 4 behandelten metaplektischen Formen. Einige dieser Formen zu kleinen Eigenwerten werden in diesem Kapitel bestimmt. In einem zweiten Abschnitt wird in Anlehnung an [6] ein Vergleich zwischen den L-Reihen getwisteter automorpher Formen und den Fourierkoeffizienten metaplektischer Formen angestellt.
\end{abstract}

\subsection{Numerische Bestimmung}

Mit den in Kapitel 4 beschriebenen Methoden war es möglich, analog zu dem Ansatz in Kapitel 3 metaplektische Formen zu bestimmen. Für die Berechnung dieser Lösungen wurde das Kollokationsfehlerquadrat der Funktion und ihrer Normalableitung an allen Punkten eines quadratischen Punktgitters mit einer Seitenlänge von $\mathrm{h}=0.04$ minimiert. Damit ergeben sich 129 Punkte auf den Viertelsechsecken und 258 Punkte auf den halben Sechsecken und damit 2322 reelle Gleichungen bei 266 reellen Unbekannten in der Fourierentwicklung um $\infty$ und 555 reellen Unbekannten in der Entwicklung um $\omega_{6}$. Die so bestimmten Lösungen sind im Verzeichnis chap5 auf der CD verfügbar. Da die Formen sich nicht mehr reell darstellen lassen, ist die Bestimmung der Eigenwerte etwas erschwert. Da es für Parameter $R$ zu einem Eigenwert von $\Delta$ Eigenfor- 
men gibt, sollten endliche Approximationen dieser Eigenformen einerseits bei einer gemeinsamen Normierung, etwa durch $A(1)=1$ (oder $i$ ), vergleichsweise kleine Absolutbeträge der Fourierkoeffizienten haben. Andererseits sollten die konvergenten Lösungen auf der ganzen Grenzfläche, und nicht nur an den Kollokationspunkten, der Gleichung $\gamma f(w)=\chi(\gamma) f(w)$ genügen.

\begin{tabular}{|c|c|c|c|c|c|c|c|}
\hline$R_{1}^{+}$ & 4.16670006 & $R_{11}^{+}$ & 8.10822342 & $R_{1}^{-}$ & 2.35733473 & $R_{11}^{-}$ & $7.31105908 *)$ \\
\hline$R_{2}^{+}$ & 5.00323536 & $R_{12}^{+}$ & $\left.8.11533888^{\dagger}\right)$ & $R_{2}^{-}$ & 3.66937112 & $R_{12}^{-}$ & 7.31105908 \\
\hline$R_{3}^{+}$ & 5.69955190 & $R_{13}^{+}$ & 8.58933465 & $R_{3}^{-}$ & 4.43105424 & $R_{13}^{-}$ & $\left.7.42064242^{*}\right)$ \\
\hline$R_{4}^{+}$ & 6.27483627 & $R_{14}^{+}$ & 8.69555537 & $R_{4}^{-}$ & 4.95453278 & $R_{14}^{-}$ & 7.75829547 \\
\hline$R_{5}^{+}$ & $\left.6.35579684^{\dagger}\right)$ & $R_{15}^{+}$ & $\left.8.75620434^{*}\right)$ & $R_{5}^{-}$ & $\left.5.37908054^{*}\right)$ & $R_{15}^{-}$ & $7.81868301^{*)}$ \\
\hline$R_{6}^{+}$ & 7.02600170 & $R_{16}^{+}$ & 9.12670677 & $R_{6}^{-}$ & 5.79889530 & $R_{16}^{-}$ & 8.16778051 \\
\hline$R_{7}^{+}$ & 7.05074345 & $R_{17}^{+}$ & 9.18650090 & $R_{7}^{-}$ & 6.05337957 & $R_{17}^{-}$ & 8.41532324 \\
\hline$R_{8}^{+}$ & 7.53669657 & $R_{18}^{+}$ & 9.33154758 & $R_{8}^{-}$ & $\left.6.45032417^{*}\right)$ & $R_{18}^{-}$ & 8.57161085 \\
\hline$R_{9}^{+}$ & $\left.7.55161848^{*}\right)$ & $R_{19}^{+}$ & 9.57233968 & $R_{9}^{-}$ & 6.72630204 & $R_{19}^{-}$ & $\left.8.71821411^{*}\right)$ \\
\hline \multirow[t]{2}{*}{$R_{10}^{+}$} & 7.92429480 & $R_{20}^{+}$ & $\left.9.69135104^{*}\right)$ & $R_{10}^{-}$ & 6.98074676 & & \\
\hline & & $\begin{array}{l}R_{21}^{+} \\
R_{22}^{+}\end{array}$ & $\begin{array}{l}9.69336704 \\
9.75906427\end{array}$ & & & & \\
\hline
\end{tabular}

*) Diese Eigenwerte haben einen Eigenraum der Dimension 2.

$\dagger$ Diese Eigenwerte stammen von $\operatorname{PSL}(2, \mathbf{Z})$.

Tabelle 5.1: Liste der kleinsten Eigenwerte für die ersten 25 geraden und 25 ungeraden Eigenfunktionen. Die Eigenwerte $R_{\nu}^{+}$bezeichnen die zu geraden, die Eigenwerte $R_{\nu}^{-}$die zu ungeraden Eigenfunktionen gehörenden Eigenwerte.

Als Indikatoren zur Eigenwertsuche wurden dementsprechend zwei Parameter gewählt: Einerseits das minimierte Fehlerquadrat, andererseits die Summe der Absolutbeträge der Fourierkoeffizienten. Es zeigt sich, daß an zu Eigenwerten gehörenden $R$ diese beiden Funktionen um viele Größenordnungen kleiner sind als außerhalb der Nachbarschaft zu diesen Punkten. Um die Eigenwertsuche graphisch darzustellen, wurden in den Grafiken 5.1-5.6 daher die Logarithmen dieser Funktionen dargestellt. Diese Logarithmen $\delta_{\log }$ und $\Sigma_{\log }$ sind allerdings wieder so normiert, daß deren Werte im Intervall [0,1] liegen. Außerdem wurde das nicht logarithmierte Fehlerquadrat in diesen Abbildungen dargestellt, allerdings mit einer Obergrenze, welche eine sinnvolle Darstellung der Kurve erlaubt (die mit $\delta^{\prime}$ bezeichnete Kurve). Das Raster für $R$ im gewählten Bereich ist 1/30, wobei bei lokalen Minima eine Minimasuche zur Bestimmung mit einer Ge- 
nauigkeit von $10^{-8}$ vorgenommen wurde. Alle Minima, welche um mehrere Größenordnungen kleiner waren als die Ausgangsfunktion, entsprachen bis auf $10^{-8}$ den in Kapitel 3 gefundenen. Die Eigenwerte heben sich, wie etwa in Abbildung 5.1 sichtbar, deutlich hervor. Die Tabelle 5.1 korrespondiert daher auch mit Tabelle 3.1. Die so bestimmten Eigenwerte wurden in den Abbildungen 5.1-5.6 durch Striche unterhalb der Graphen angedeutet.

Die Tabellen 5.2-5.5 sollen die errechneten Werte für einige beispielhafte $R$ darstellen. Dabei sind in den sechs Spalten jeweils die Fourierentwicklungen sowohl einer metaplektischen, als auch einer korrespondierenden automorphen Form abgebildet. Dazu werden in der ersten Spalte dieser Tabellen die 55 betragsmäßig kleinsten $n$ aus der Entwicklung nach $\left(4.1^{\prime \prime \prime}\right)$ und in der zweiten Spalte die dazu gehörenden $A(n)$ aufgelistet, in der dritten dann die 55 betragsmäßig kleinsten $n$ aus der Entwicklung nach $\left(4.2^{\prime \prime \prime}\right)$ mit den entsprechenden $B(n)$ in der vierten Spalte. Die korrespondierende Approximation an eine automorphe Form findet sich in den letzten beiden Spalten, wobei die fünfte Spalte die 55 betragsmäßig kleinsten $p$, mit $p$ prim oder $p=1$, auflistet, und die sechste Spalte die dazu gehörenden Fourierkoeffizienten aus der Entwicklung (3.1).

Die entsprechenden Tabellen für alle 50 Eigenwerte finden sich als Postscript-Datei auf der beigelegten CD im Verzeichnis DOC.

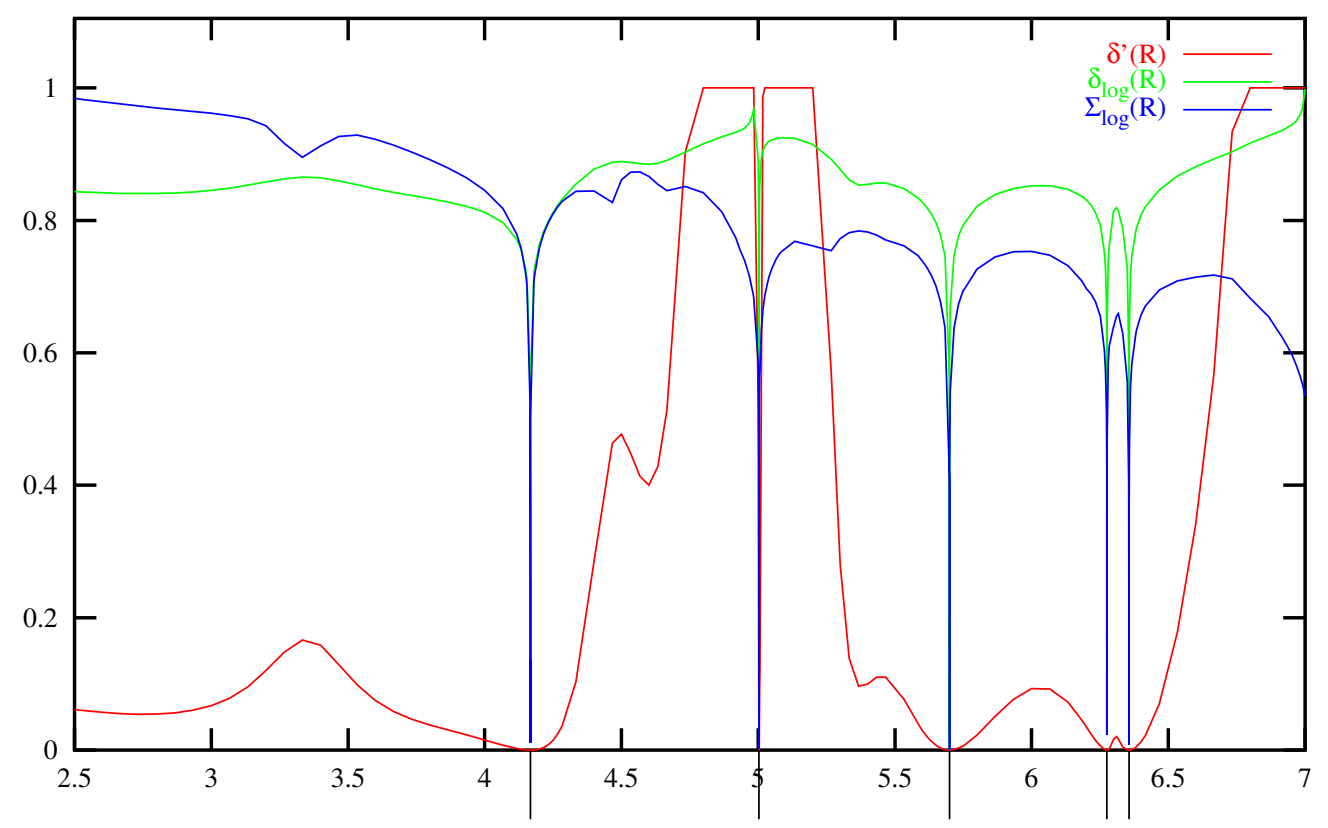

Abbildung 5.1: Die ersten fünf Eigenwerte bei geraden Eigenfunktionen 


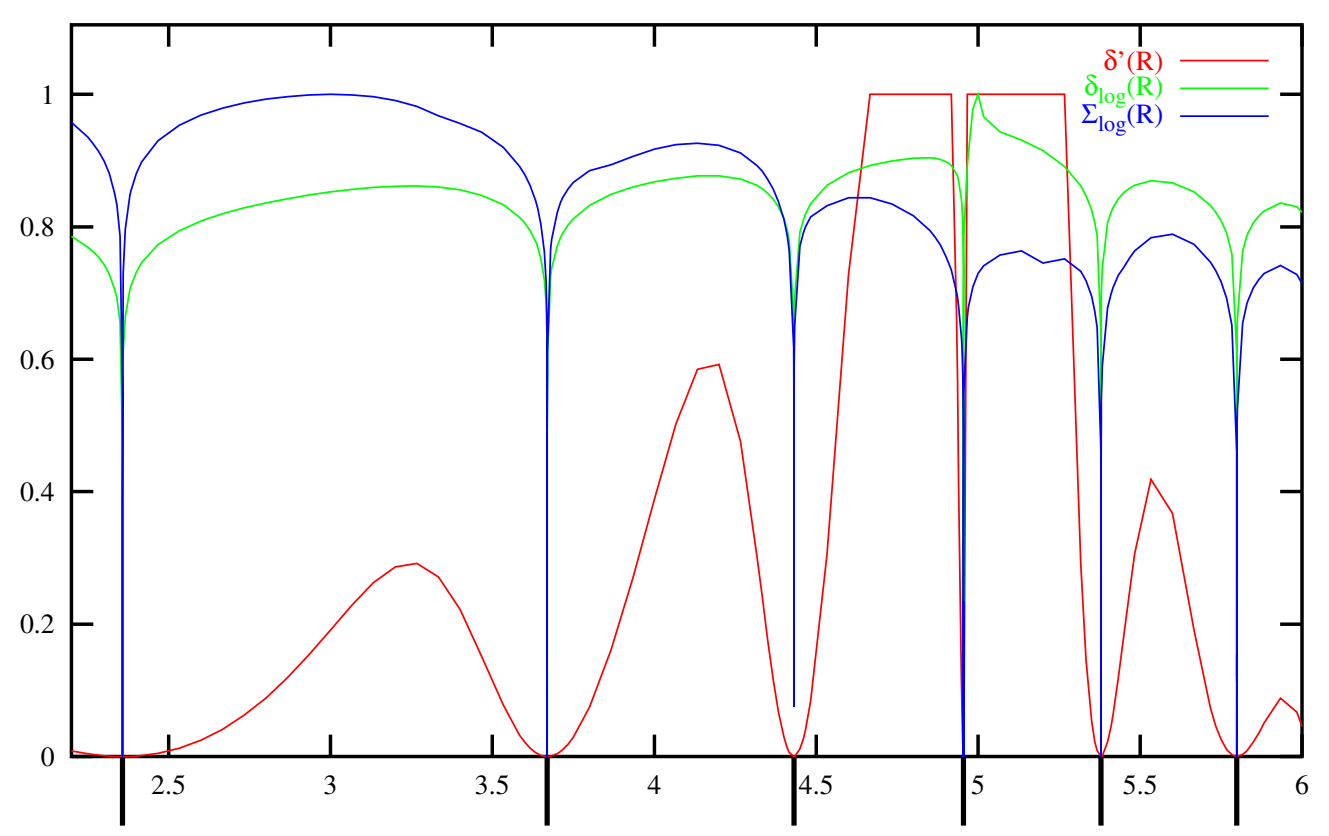

Abbildung 5.2: Die ersten 6 Eigenwerte bei ungeraden Eigenfunktionen

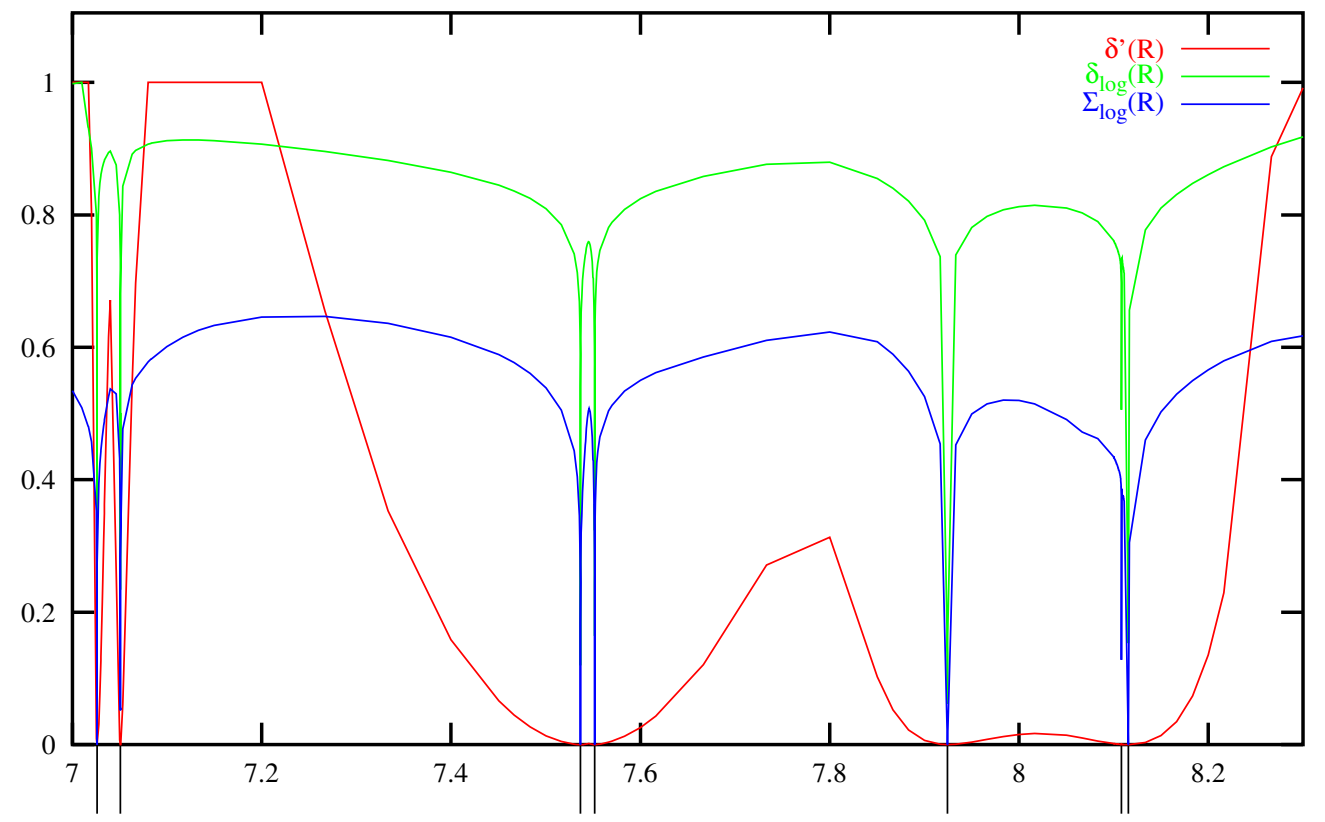

Abbildung 5.3: Eigenwertsuche für gerade Eigenfunktionen $(R \in[7,8.3])$ 


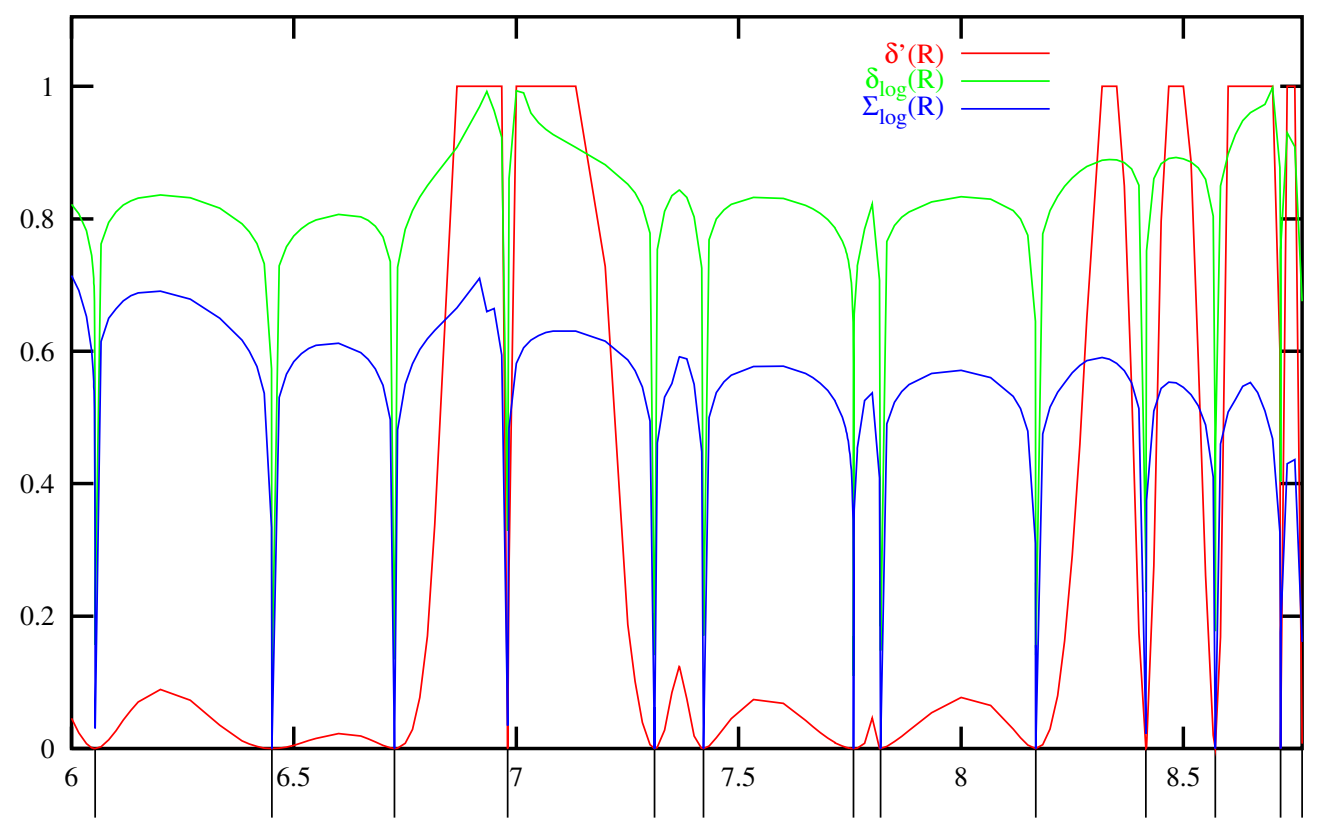

Abbildung 5.4: Eigenwertsuche für ungerade Eigenfunktionen $(R \in[6,8.8])$

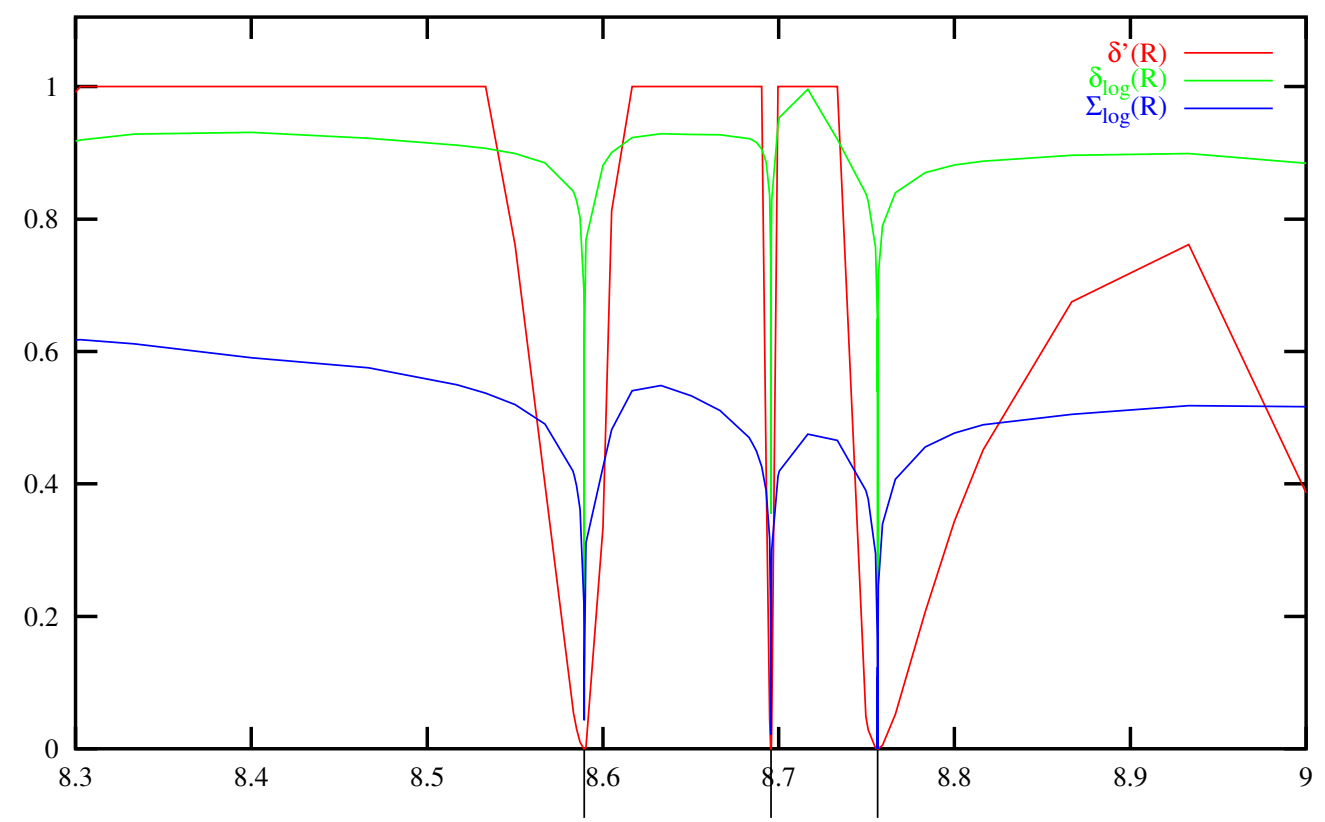

Abbildung 5.5: Eigenwertsuche für gerade Eigenfunktionen $(R \in[8.3,9])$ 


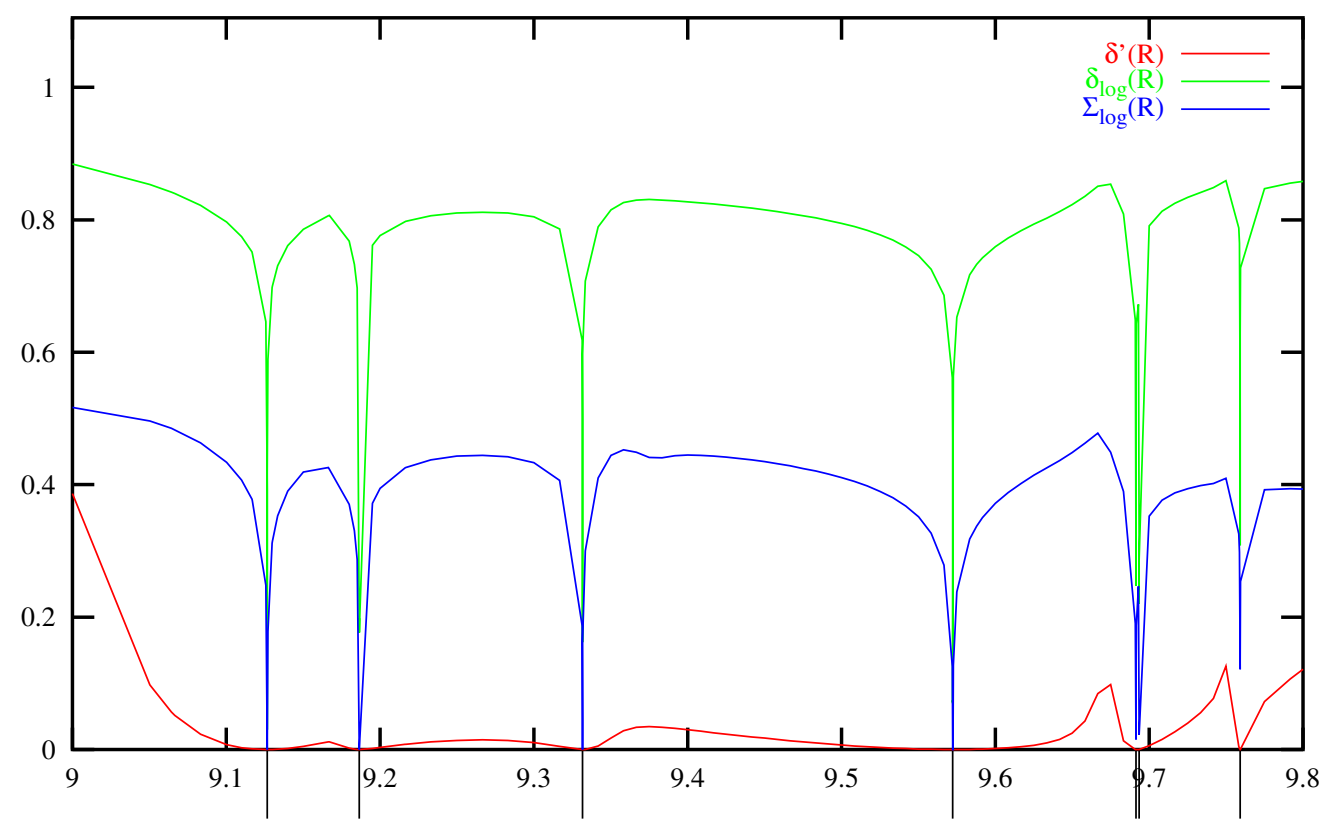

Abbildung 5.6: Eigenwertsuche für gerade Eigenfunktionen $(R \in[9,9.8])$ 


\begin{tabular}{|c|c|c|c|c|c|}
\hline$n$ & $A(n)$ wie $\left(4.1^{\prime \prime \prime}\right)$ & $n$ & $B(n)$ wie $\left(4.2^{\prime \prime \prime}\right)$ & $p$ & $c(p)$ wie $(3.1)$ \\
\hline-1 & $+1.00000000 i$ & $-1-2 \omega$ & $-2.19324998 i$ & 1 & 1.0000000000000000000000000 \\
\hline-2 & $+1.98853169 i$ & $-1-5 \omega$ & $-0.58809743-1.82778476 i$ & $2+\omega$ & 0.0000000000000000000000000 \\
\hline $1+3 \omega$ & $0.07452169-3.92727249 i$ & $-4-8 \omega$ & $-0.27951420 i$ & 2 & -1.2235534628458702437086909 \\
\hline-3 & $-0.07119763 i$ & $2-2 \omega$ & $-0.70094657 i$ & $3+2 \omega$ & -0.8396690036824385585311796 \\
\hline$+3 \omega$ & $-0.25404645-0.74124813 i$ & $2-5 \omega$ & $0.67780005-0.12276449 i$ & $3+\omega$ & 0.8396690036824385585311796 \\
\hline$-1+3 \omega$ & $0.49986192+2.71264125 i$ & $-1-8 \omega$ & $-0.61162970+0.70488538 i$ & $4+3 \omega$ & 5772733089604642858 \\
\hline-4 & $+0.57923551 i$ & $-4-11 \omega$ & $-0.34707538-0.23975533 i$ & $4+\omega$ & 1.0766645772733089604642858 \\
\hline$-2+3 \omega$ & $-0.72147949-0.82090942 i$ & $2-8 \omega$ & $-0.16332892+0.13981308 i$ & $5+3 \omega$ & 1.1986497563053090515387595 \\
\hline-5 & $+0.7354845 i$ & $-7-14 \omega$ & $-0.14765153 i$ & $5+2 \omega$ & -1.1986497563053090515387595 \\
\hline $3+6 \omega$ & 0.4873889 & $5-5 \omega$ & $+0.24054344 i$ & 5 & 1.9656716789984966540935762 \\
\hline$-3+3 \omega$ & $0.7076121-1.9283230 i$ & $-1-11 \omega$ & $0.29375983-0.03139369 i$ & $6+5 \omega$ & -0.0747021743404604023776511 \\
\hline $2+6 \omega$ & $-1.1629465-0.1933623 i$ & $-4-14 \omega$ & $-0.55045911+0.55453968 i$ & $6+\omega$ & 0.0747021743404604023776511 \\
\hline $1+6 \omega$ & $1.2461858-0.6875939 i$ & $5-8 \omega$ & $0.08249633+0.33651849 i$ & $7+4 \omega$ & -0.6291362258139189374553433 \\
\hline-6 & $+0.0562242 i$ & $2-11 \omega$ & $0.21760227-0.38154632 i$ & $7+3 \omega$ & 0.6291362258139189374553433 \\
\hline$+6 \omega$ & $-0.6118365-0.0234731 i$ & $-7-17 \omega$ & $0.23947737-0.40154335 i$ & $7+6 \omega$ & -0.7161184181841670489200989 \\
\hline$-4+3 \omega$ & $-0.4879604-0.0237302 i$ & $-1-14 \omega$ & $0.28924596-0.06243164 i$ & $7+\omega$ & 89200989 \\
\hline$-1+6 \omega$ & $-0.5002854+0.9491294 i$ & $-4-17 \omega$ & $-0.04543784+0.26267790 i$ & $9+5 \omega$ & -0.0400314581172724648718048 \\
\hline-7 & $+0.4623254 i$ & $5-11 \omega$ & $0.50526208-0.66627873 i$ & $9+4 \omega$ & 0.0400314581172724648718048 \\
\hline$-5+3 \omega$ & $0.4425673+0.1603353 i$ & $8-8 \omega$ & $-0.2478264 i$ & $9+7 \omega$ & 1.0289393642788992268654192 \\
\hline$-2+6 \omega$ & $1.4696325+0.3907568 i$ & $-10-20 \omega$ & $+0.2244428 i$ & $9+2 \omega$ & -1.0289393642788992268654192 \\
\hline $4+9 \omega$ & $-0.437549-1.297784 i$ & $2-14 \omega$ & $-0.3091244+0.3518803 i$ & $9+8 \omega$ & 462806 \\
\hline$-6+3 \omega$ & $-0.485196+0.723843 i$ & $-7-20 \omega$ & $-0.1735470-0.2053396 i$ & $9+\omega$ & -0.2882366827806330305462806 \\
\hline$-3+6 \omega$ & $-1.778176-0.508004 i$ & $-1-17 \omega$ & $0.1481252+0.4650446 i$ & $10+7 \omega$ & 0.2625974796957001571878541 \\
\hline $3+9 \omega$ & $0.906945-0.082233 i$ & $-4-20 \omega$ & $-0.2490269-0.2465397 i$ & $10+3 \omega$ & -0.2625974796957001571878541 \\
\hline-8 & $-0.401462 i$ & $8-11 \omega$ & $-0.3347347-0.2183459 i$ & $11+8 \omega$ & 0.5269113060239121948192348 \\
\hline & $-0.395988+0.048499 i$ & & $-0.2388649+0.2118882 i$ & & ה00000101040100 \\
\hline $1+9 \omega$ & $-0.775006-0.206495 i$ & $2-17 \omega$ & $0.0423799-0.2399815 i$ & $1+9 \omega$ & 0.3208466286257447915569120 \\
\hline$-4+6 \omega$ & $0.786821+0.294023 i$ & $-10-23 \omega$ & $0.341670-0.181120 i$ & $1+2 \omega$ & -0.3208466286257447915569120 \\
\hline$-7+3 \omega$ & $0.04577-0.02567 i$ & $-1-20 \omega$ & $0.217220+0.350230 i$ & $12+7 \omega$ & 1.3208113104927926775602717 \\
\hline-9 & $-0.15771 i$ & $-7-23 \omega$ & $-0.173463-0.252880 i$ & $12+5 \omega$ & -1.3208113104927926775602717 \\
\hline$+9 \omega$ & $1.08852+0.62057 i$ & $8-14 \omega$ & $-0.166760+0.419658 i$ & 11 & 5997892 \\
\hline$-1+9 \omega$ & $-0.01501-0.37266 i$ & $11-11 \omega$ & $-0.040374 i$ & $3+7 \omega$ & -1.3672206662566281969 \\
\hline$-5+6 \omega$ & $0.87237-0.48928 i$ & $-4-23 \omega$ & $-0.296558-0.031590 i$ & & 1.36722066625662819 \\
\hline$-8+3 \omega$ & $0.4768-0.4398 i$ & $5-17 \omega$ & $-0.140907+0.255106 i$ & $13+10 \omega$ & 0.6717098722328782247921610 \\
\hline-10 & $+0.1570 i$ & $-13-26 \omega$ & $+0.080612 i$ & $13+3 \omega$ & -0.6717098722328782247921610 \\
\hline$-2+9 \omega$ & $-0.3362-0.4894 i$ & $-10-26 \omega$ & $-0.143389+0.229573 i$ & $14+9 \omega$ & 0.5217481093920599303554359 \\
\hline$-6+6 \omega$ & $-1.0462-0.2594 i$ & $2-20 \omega$ & $0.365436+0.026241 i$ & & -0.521748109392059930 \\
\hline $6+12 \omega$ & -0.1558 & $-7-26 \omega$ & $0.198767-0.230331 i$ & $13+12 \omega$ & -0.6155827601237021618307143 \\
\hline $5+12 \omega$ & $0.4290-0.4$ & & $0.09075-0.12669 i$ & & 0.6155827601237021618307143 \\
\hline $4+12 \omega$ & $-0.5135+0.2935 i$ & $11-14 \omega$ & $-0.01039+0.14657 i$ & $14+11 \omega$ & 1.6897589220099385689575848 \\
\hline$-9+3 \omega$ & $-0.2285-0.3322 i$ & $8-17 \omega$ & $0.23227+0.07373 i$ & $14+3 \omega$ & -1.6897589220099385689575848 \\
\hline$-3+9 \omega$ & $-0.5806-0.1471 i$ & $5-20 \omega$ & $0.08944+0.08377 i$ & $+11 \omega$ & -1.5413461353912764214651622 \\
\hline $3+12 \omega$ & $0.520+0.500 i$ & $-13-29 \omega$ & $0.08830+0.10095 i$ & $5+4 \omega$ & 1.5413461353912764214651622 \\
\hline-11 & $+0.414 i$ & $-4-26 \omega$ & $-0.07253-0.26442 i$ & $16+9 \omega$ & 0.0836855551834201409734036 \\
\hline $2+12 \omega$ & $-0.321+0.740 i$ & $2-23 \omega$ & $0.00058-0.15941 i$ & $16+7 \omega$ & -0.0836855551834201409734036 \\
\hline$-7+6 \omega$ & $0.032+0.245 i$ & $-10-29 \omega$ & $0.02385+0.08308 i$ & $15+13 \omega$ & -0.8585146214484064430323731 \\
\hline $1+12 \omega$ & $-0.313+0.478 i$ & $-7-29 \omega$ & $0.07653+0.13865 i$ & $2 \omega$ & 0.8585146214484064430323731 \\
\hline$-4+9 \omega$ & $0.357+0.511 i$ & $-1-26 \omega$ & $0.03427-0.19519 i$ & $15+14 \omega$ & 1.1141042534449374581398225 \\
\hline$-10+3 \omega$ & $-0.338-0.628 i$ & $11-17 \omega$ & $-0.00094-0.27637 i$ & $15+\omega$ & -1.1141042534449374581398225 \\
\hline-12 & $+0.625 i$ & $14-14 \omega$ & $-0.02314 i$ & $17+11 \omega$ & 0.3832536190747926045462154 \\
\hline$+12 \omega$ & $0.317-0.566 i$ & $8-20 \omega$ & $-0.08334-0.29188 i$ & $17+6 \omega$ & -0.3832536190747926045462154 \\
\hline$-8+6 \omega$ & $-0.135-0.248 i$ & $-16-32 \omega$ & $-0.03851 i$ & & 0.8418532718222818594056584 \\
\hline$-5+9 \omega$ & $0.399+0.599 i$ & $5-23 \omega$ & $0.03864-0.04696 i$ & $17+5 \omega$ & -0.8418532718222818594056584 \\
\hline$-1+12 \omega$ & $0.30-0.61 i$ & $-13-32 \omega$ & $-0.13309+0.00236 i$ & $16+15 \omega$ & -0.1911190905480637616337007 \\
\hline$-11+3 \omega$ & $0.44+0.60 i$ & $-4-29 \omega$ & $-0.04119+0.11634 i$ & $16+\omega$ & 0.1911190905480637616337007 \\
\hline
\end{tabular}

Tabelle 5.2: Metaplektische und automorphe Fourierentwicklung für $R=R_{3}^{-}$ 


\begin{tabular}{|c|c|c|c|c|c|}
\hline$n$ & $A(n)$ wie $\left(4.1^{\prime \prime \prime}\right)$ & $n$ & $B(n)$ wie $\left(4.2^{\prime \prime \prime}\right)$ & $p$ & $c(p)$ wie $(3.1)$ \\
\hline-1 & 1.00000000 & $-1-2 \omega$ & -0.02203438 & 1 & 1.0000000000000000000000000 \\
\hline-2 & 0.19753432 & $-1-5 \omega$ & $-0.09316210-0.17007450 i$ & $2+\omega$ & -0.3982427616050333073122704 \\
\hline $1+3 \omega$ & $-0.75104312+0.48084064 i$ & $-4-8 \omega$ & -0.12948250 & 2 & 1.7942189271757078747398505 \\
\hline-3 & 0.20735857 & $2-2 \omega$ & -0.04431570 & $3+2 \omega$ & -1.4113602257120067654665920 \\
\hline$+3 \omega$ & $-0.57077504-0.14518063 i$ & $2-5 \omega$ & $-0.28179907-0.01582528 i$ & $3+\omega$ & -1.4113602257120067654665920 \\
\hline$-1+3 \omega$ & $-0.11728776-0.20521140 i$ & $-1-8 \omega$ & $-0.02360498-0.09917957 i$ & $4+3 \omega$ & 0.0618204480220294091926918 \\
\hline-4 & -0.01653356 & $-4-11 \omega$ & $0.03972401+0.14804766 i$ & $4+\omega$ & 0.0618204480220294091926918 \\
\hline$-2+3 \omega$ & $0.26981417+0.39443861 i$ & $2-8 \omega$ & $0.01079305+0.13380758 i$ & $5+3 \omega$ & -0.4660763359376885205746926 \\
\hline-5 & -0.0273683 & $-7-14 \omega$ & 0.00148914 & $5+2 \omega$ & -0.4660763359376885205746926 \\
\hline $3+6 \omega$ & -0.0717453 & $5-5 \omega$ & 0.03983225 & 5 & 1.7720451827660270106344506 \\
\hline$-3+3 \omega$ & $0.0155959-0.2739663 i$ & $-1-11 \omega$ & $0.03941797+0.04439566 i$ & $6+5 \omega$ & 0.5832642874218084144556346 \\
\hline $2+6 \omega$ & $0.0422523-0.2017915 i$ & $-4-14 \omega$ & $-0.02777415+0.05415558 i$ & $6+\omega$ & 0.5832642874218084144556346 \\
\hline $1+6 \omega$ & $-0.0207612-0.1932631 i$ & $5-8 \omega$ & $-0.18591968-0.07025973 i$ & $7+4 \omega$ & 0.8663316985604420612982511 \\
\hline-6 & 0.1600859 & $2-11 \omega$ & $-0.07582779-0.09242919 i$ & $7+3 \omega$ & 0.8663316985604420612982511 \\
\hline$+6 \omega$ & $-0.0380190+0.2020192 i$ & $-7-17 \omega$ & $-0.06091212+0.08774762 i$ & $7+6 \omega$ & -0.2684602870113338810223403 \\
\hline$-4+3 \omega$ & $-0.0213469+0.0182935 i$ & $-1-14 \omega$ & $-0.06230725+0.06662617 i$ & $7+\omega$ & -0.2684602870113338810223403 \\
\hline$-1+6 \omega$ & $6257+0.0214140 i$ & $-4-17 \omega$ & $0.04122149+0.08186470 i$ & $9+5 \omega$ & -0.7423759336410702392839332 \\
\hline-7 & 0.1637039 & $5-11 \omega$ & $-0.02102716+0.05234165 i$ & $9+4 \omega$ & -0.7423759336410702392839332 \\
\hline$-5+3 \omega$ & $0.1491156+0.0180253 i$ & $8-8 \omega$ & 0.0005977 & $9+7 \omega$ & 0.2650251710776058500370574 \\
\hline$-2+6 \omega$ & $0.2245632+0.1273898 i$ & $-10-20 \omega$ & 0.0295369 & $9+2 \omega$ & 0.2650251710776058500370574 \\
\hline $4+9 \omega$ & $-0.101102-0.033183 i$ & & $-0.0126420-0.0122157 i$ & $9+8 \omega$ & 027898 \\
\hline$-6+3 \omega$ & $0.133721-0.009631 i$ & $-7-20 \omega$ & $0.0238929+0.0010242 i$ & $9+\omega$ & 0.5626799993421531322027898 \\
\hline$-3+6 \omega$ & $0.075219-0.058186 i$ & $-1-17 \omega$ & $0.0205171+0.1491035 i$ & $10+7 \omega$ & 0.0432204487755142415369701 \\
\hline $3+9 \omega$ & $-0.104344-0.022903 i$ & $-4-20 \omega$ & $-0.0535139-0.0241574 i$ & $10+3 \omega$ & 0.0432204487755142415369701 \\
\hline-8 & 0.199586 & $8-11 \omega$ & $0.0358559-0.0104735 i$ & $11+8 \omega$ & 0.8444612600497549890036120 \\
\hline $2+9 \omega$ & $-0.026677-0.057823 i$ & $5-14 \omega$ & $0.0277723-0.0779978 i$ & $11+3 \omega$ & 0.8444612600497549890036120 \\
\hline $1+9 \omega$ & $-0.026755-0.053180 i$ & $2-17 \omega$ & $0.0043916-0.0109995 i$ & $11+9 \omega$ & -1.6624248590391364239409732 \\
\hline$-4+6 \omega$ & $0.154636-0.202395 i$ & $-10-23 \omega$ & $0.077843+0.030708 i$ & $11+2 \omega$ & -1.6624248590391364239409732 \\
\hline$-7+3 \omega$ & $0.07454+0.13463 i$ & $-1-20 \omega$ & $0.006086+0.014646 i$ & $12+7 \omega$ & 1.2570516944483100091724279 \\
\hline-9 & 0.07150 & $-7-23 \omega$ & $-0.049801+0.029695 i$ & $12+5 \omega$ & 1.2570516944483100091724279 \\
\hline$+9 \omega$ & & & $0.065348+0.036174 i$ & & 917336 \\
\hline$-1+9 \omega$ & $-0.11562+0.12968 i$ & $11-11 \omega$ & 0.039672 & $13+7 \omega$ & -0.1752390074481811020312226 \\
\hline$-5+6 \omega$ & $-0.12593-0.01303 i$ & $-4-23 \omega$ & $-0.030827-0.008765 i$ & $13+6 \omega$ & -0.1752390074481811020312226 \\
\hline$-8+3 \omega$ & $-0.0053+0.0003 i$ & $5-17 \omega$ & $-0.001145-0.005118 i$ & $13+10 \omega$ & -1.5615602771356498854063657 \\
\hline-10 & 0.0078 & $-13-26 \omega$ & 0.023545 & $13+3 \omega$ & -1.5615602771356498854063657 \\
\hline$-2+9 \omega$ & $-0.0728+0.0223 i$ & $-10-26 \omega$ & $-0.026479+0.064131 i$ & $14+9 \omega$ & 221609646 \\
\hline$-6+6 \omega$ & $0.0076+0.1346 i$ & $2-20 \omega$ & $0.014613-0.039855 i$ & $14+5 \omega$ & 8869221609646 \\
\hline $6+12 \omega$ & -0.0053 & $-7-26 \omega$ & $0.030133-0.004433 i$ & $13+12 \omega$ & 0.6231087401911425714294114 \\
\hline $5+12 \omega$ & $0.2389-0.0896 i$ & $-1-23 \omega$ & $-0.04625-0.02645 i$ & $13+\omega$ & 0.6231087401911425714294114 \\
\hline $4+12 \omega$ & $0.0155-0.0932 i$ & $11-14 \omega$ & $-0.05369+0.04057 i$ & $14+11 \omega$ & 1.0482590465436635772056915 \\
\hline$-9+3 \omega$ & $0.0404-0.1025 i$ & $8-17 \omega$ & $-0.03825-0.01998 i$ & & 1.0482590465436635772 \\
\hline$-3+9 \omega$ & $-0.1949-0.1121 i$ & $5-20 \omega$ & $-0.04339+0.05214 i$ & $+11 \omega$ & 1.2258396101453748321612865 \\
\hline $3+12 \omega$ & $0.148+0.114 i$ & $-13-29 \omega$ & $-0.00828-0.00152 i$ & $15+4 \omega$ & 1.2258396101453748321612865 \\
\hline-11 & 0.073 & $-4-26 \omega$ & $0.03263+0.00991 i$ & $16+9 \omega$ & 1.1730874620483679947036207 \\
\hline $2+12 \omega$ & $-0.063+0.045 i$ & $2-23 \omega$ & $0.01973-0.02297 i$ & $16+7 \omega$ & 1.1730874620483679947036207 \\
\hline$-7+6 \omega$ & $-0.117+0.050 i$ & $-10-29 \omega$ & $0.00357-0.04100 i$ & $15+13 \omega$ & -1.2585491763055633350653067 \\
\hline $1+12 \omega$ & $0.058+0.079 i$ & $-7-29 \omega$ & $0.05014+0.00611 i$ & $15+2 \omega$ & -1.2585491763055633350653067 \\
\hline$-4+9 \omega$ & $-0.134+0.038 i$ & $-1-26 \omega$ & $-0.04548-0.03697 i$ & $15+14 \omega$ & -0.3167363635781918528294216 \\
\hline$-10+3 \omega$ & $0.119-0.119 i$ & $11-17 \omega$ & $-0.05570+0.03466 i$ & $15+\omega$ & -0.3167363635781918528294216 \\
\hline-12 & 0.050 & $14-14 \omega$ & -0.01441 & $17+11 \omega$ & 1.2195503546691110937039779 \\
\hline$+12 \omega$ & $-0.052-0.099 i$ & $8-20 \omega$ & $0.01176-0.01495 i$ & $17+6 \omega$ & 1.2195503546691110937039779 \\
\hline$-8+6 \omega$ & $-0.021-0.013 i$ & $-16-32 \omega$ & -0.00925 & $17+12 \omega$ & -0.3280105530101637731837869 \\
\hline$-5+9 \omega$ & $0.075-0.067 i$ & $5-23 \omega$ & $-0.00820-0.05705 i$ & $17+5 \omega$ & -0.3280105530101637731837869 \\
\hline$-1+12 \omega$ & $0.05-0.11 i$ & $-13-32 \omega$ & $-0.04742+0.00882 i$ & $16+15 \omega$ & 0.7276397332642290494811938 \\
\hline$-11+3 \omega$ & $0.09+0.03 i$ & $-4-29 \omega$ & $-0.01409-0.02114 i$ & $16+\omega$ & 0.7276397332642290494811938 \\
\hline
\end{tabular}

Tabelle 5.3: Metaplektische und automorphe Fourierentwicklung für $R=R_{6}^{+}$ 


\begin{tabular}{|c|c|c|c|c|c|}
\hline$n$ & $A(n)$ wie $\left(4.1^{\prime \prime \prime}\right)$ & $n$ & $B(n)$ wie $\left(4.2^{\prime \prime \prime}\right)$ & $p$ & $c(p)$ wie $(3.1)$ \\
\hline-1 & 1.00000000 & $-1-2 \omega$ & 0.27444427 & 1 & 1.000000000000000000 \\
\hline-2 & -0.66401360 & $-1-5 \omega$ & $-0.17754818-0.30095006 i$ & $2+\omega$ & 0.974455153242599482 \\
\hline $1+3 \omega$ & $0.26694687-0.31698719 i$ & $-4-8 \omega$ & 0.05235499 & 2 & -1.175006293091341487 \\
\hline-3 & 0.18694286 & $2-2 \omega$ & -0.11995261 & $3+2 \omega$ & -0.731596565810402583 \\
\hline$+3 \omega$ & $0.11361553-0.46439154 i$ & $2-5 \omega$ & $-0.01572544+0.13159139 i$ & $3+\omega$ & 0.587080090761923263 \\
\hline$-1+3 \omega$ & $0.77326353+0.48443170 i$ & $-1-8 \omega$ & $-0.10320984-0.04449359 i$ & $4+3 \omega$ & -1.063828009303246491 \\
\hline-4 & 0.03416533 & $-4-11 \omega$ & $0.07904803+0.27265018 i$ & $4+\omega$ & -0.647611327465178281 \\
\hline$-2+3 \omega$ & $0.11622323-0.14816889 i$ & $2-8 \omega$ & $0.00272078+0.04642524 i$ & $5+3 \omega$ & 0.162243908973960715 \\
\hline-5 & -0.0954068 & $-7-14 \omega$ & -0.08441362 & $5+2 \omega$ & -0.171927985296005837 \\
\hline $3+6 \omega$ & 0.1265463 & $5-5 \omega$ & -0.00175397 & 5 & 0.463909856599805109 \\
\hline$-3+3 \omega$ & $-0.3900161-0.1360981 i$ & $-1-11 \omega$ & $-0.02505339+0.18803190 i$ & $6+5 \omega$ & -1.037948840088401175 \\
\hline $2+6 \omega$ & $0.0462401+0.1173848 i$ & $-4-14 \omega$ & $0.13789201-0.03302989 i$ & $6+\omega$ & -0.492467664482579292 \\
\hline $1+6 \omega$ & $-0.1407989+0.4049142 i$ & $5-8 \omega$ & $0.04537850+0.06286987 i$ & $7+4 \omega$ & 0.473628076670323690 \\
\hline-6 & -0.0212563 & $2-11 \omega$ & $-0.00627909+0.14310712 i$ & $7+3 \omega$ & -0.161982361949622732 \\
\hline$+6 \omega$ & $0.0968724-0.0922022 i$ & $-7-17 \omega$ & $0.08443317-0.01158343 i$ & $7+6 \omega$ & -0.679178644943579004 \\
\hline$-4+3 \omega$ & $-0.2616730+0.0917271 i$ & $-1-14 \omega$ & $-0.05445215+0.11269841 i$ & $7+\omega$ & -0.163180256243435556 \\
\hline$-1+6 \omega$ & $0.1020378+0.0376744 i$ & $-4-17 \omega$ & $-0.02668161-0.02685669 i$ & $9+5 \omega$ & 0.167705141445407517 \\
\hline-7 & -0.1879538 & $5-11 \omega$ & $-0.00812213+0.11039456 i$ & $9+4 \omega$ & 0.213111184872026280 \\
\hline$-5+3 \omega$ & $-0.1471923+0.0469019 i$ & $8-8 \omega$ & -0.0253151 & $9+7 \omega$ & -0.402412369132929498 \\
\hline$-2+6 \omega$ & $0.3397962-0.0293872 i$ & $-10-20 \omega$ & 0.0256106 & $9+2 \omega$ & -0.121364972205079390 \\
\hline $4+9 \omega$ & $0.124695+0.019673 i$ & $2-14 \omega$ & $0.1308124-0.0271602 i$ & $9+8 \omega$ & 0.288920321792852548 \\
\hline$-6+3 \omega$ & $-0.235586+0.018949 i$ & $-7-20 \omega$ & $-0.0202389-0.0747554 i$ & $9+\omega$ & -0.267309615803910291 \\
\hline$-3+6 \omega$ & $0.132078-0.247427 i$ & $-1-17 \omega$ & $-0.0650879-0.0406885 i$ & $10+7 \omega$ & -0.164387727466458335 \\
\hline $3+9 \omega$ & $0.032499+0.013754 i$ & $-4-20 \omega$ & $-0.0611111-0.0488460 i$ & $10+3 \omega$ & -1.148463221948321295 \\
\hline-8 & -0.063874 & $8-11 \omega$ & $0.0572598+0.0485218 i$ & $11+8 \omega$ & -0.365805987476726974 \\
\hline $2+9 \omega$ & $-0.131388-0.076193 i$ & $5-14 \omega$ & $0.0026814+0.0266075 i$ & $11+3 \omega$ & -0.571100613671304723 \\
\hline $1+9 \omega$ & $0.106369+0.056320 i$ & $2-17 \omega$ & $0.0655857-0.0058360 i$ & $11+9 \omega$ & 0.689383912996105084 \\
\hline$-4+6 \omega$ & $-0.011172+0.082970 i$ & $-10-23 \omega$ & $-0.007608-0.032799 i$ & $11+2 \omega$ & 0.796582846864461617 \\
\hline$-7+3 \omega$ & $-0.00346-0.07008 i$ & $-1-20 \omega$ & $0.050207-0.036717 i$ & $12+7 \omega$ & 0.488137746036444524 \\
\hline-9 & -0.01829 & $-7-23 \omega$ & $-0.003827-0.103229 i$ & $12+5 \omega$ & 0.143193304870476034 \\
\hline$+9 \omega$ & $-0.20342+0.26584 i$ & $8-14 \omega$ & $-0.063326+0.014476 i$ & 11 & -0.400259936578718722 \\
\hline$-1+9 \omega$ & $-0.09400-0.07303 i$ & $11-11 \omega$ & -0.044293 & $13+7 \omega$ & 0.429058923187070027 \\
\hline$-5+6 \omega$ & $0.18267+0.09033 i$ & $-4-23 \omega$ & $0.033720+0.005700 i$ & $13+6 \omega$ & 1.011333545701487928 \\
\hline$-8+3 \omega$ & $0.2835+0.0731 i$ & $5-17 \omega$ & $-0.017101-0.000541 i$ & $13+10 \omega$ & 0.592652032294269351 \\
\hline-10 & -0.1052 & $-13-26 \omega$ & 0.013566 & $13+3 \omega$ & 1.133138316473920832 \\
\hline$-2+9 \omega$ & $0.0115-0.0735 i$ & $-10-26 \omega$ & $0.005417+0.043782 i$ & $14+9 \omega$ & 1.147176248977683379 \\
\hline$-6+6 \omega$ & $0.1443+0.0705 i$ & $2-20 \omega$ & $0.008703+0.080104 i$ & $14+5 \omega$ & 0.356660081214303679 \\
\hline $6+12 \omega$ & -0.0979 & $-7-26 \omega$ & $0.009323-0.006725 i$ & $13+12 \omega$ & -0.008354141622158139 \\
\hline $5+12 \omega$ & $0.0110+0.0348 i$ & $-1-23 \omega$ & $-0.04974-0.01317 i$ & $13+\omega$ & 0.615044030387230990 \\
\hline $4+12 \omega$ & $0.0078-0.1164 i$ & $11-14 \omega$ & $-0.00057-0.03114 i$ & $14+11 \omega$ & 0.140129666902732691 \\
\hline$-9+3 \omega$ & $0.1673-0.0965 i$ & $8-17 \omega$ & $-0.04435-0.00125 i$ & $14+3 \omega$ & -0.237701658270889518 \\
\hline$-3+9 \omega$ & $0.0697-0.1249 i$ & $5-20 \omega$ & $-0.06310-0.00801 i$ & $15+11 \omega$ & 0.133462415796061134 \\
\hline $3+12 \omega$ & $-0.007+0.059 i$ & $-13-29 \omega$ & $-0.04472-0.00641 i$ & $15+4 \omega$ & -0.647939068747701437 \\
\hline-11 & 0.040 & $-4-26 \omega$ & $-0.00107+0.05205 i$ & $16+9 \omega$ & 0.157244674380075979 \\
\hline $2+12 \omega$ & $-0.078-0.096 i$ & $2-23 \omega$ & $-0.03204-0.04397 i$ & $16+7 \omega$ & 0.516426988448303458 \\
\hline$-7+6 \omega$ & $0.142+0.002 i$ & $-10-29 \omega$ & $0.02831+0.06345 i$ & $15+13 \omega$ & 0.655717068568996562 \\
\hline $1+12 \omega$ & $0.148+0.081 i$ & $-7-29 \omega$ & $-0.00591-0.01394 i$ & $15+2 \omega$ & 0.567307922226467313 \\
\hline$-4+9 \omega$ & $0.110+0.005 i$ & $-1-26 \omega$ & $-0.01116+0.08578 i$ & $15+14 \omega$ & 1.089724039351992119 \\
\hline$-10+3 \omega$ & $0.077-0.063 i$ & $11-17 \omega$ & $-0.06920+0.03032 i$ & $15+\omega$ & 1.253025582852597562 \\
\hline-12 & 0.017 & $14-14 \omega$ & 0.01858 & $17+11 \omega$ & 0.055818843662576444 \\
\hline$+12 \omega$ & $-0.071-0.106 i$ & $8-20 \omega$ & $0.05714+0.08406 i$ & $17+6 \omega$ & 0.310059537326260739 \\
\hline$-8+6 \omega$ & $0.076-0.084 i$ & $-16-32 \omega$ & 0.00904 & $17+12 \omega$ & 0.906460595589592839 \\
\hline$-5+9 \omega$ & $-0.021+0.105 i$ & $5-23 \omega$ & $0.03907-0.02616 i$ & $17+5 \omega$ & 0.505609603901547895 \\
\hline$-1+12 \omega$ & $-0.09+0.12 i$ & $-13-32 \omega$ & $-0.02525+0.01757 i$ & $16+15 \omega$ & -0.324366296855912582 \\
\hline$-11+3 \omega$ & $0.09+0.03 i$ & $-4-29 \omega$ & $-0.02478-0.01962 i$ & $16+\omega$ & 0.634707757489658556 \\
\hline
\end{tabular}

Tabelle 5.4: Metaplektische und automorphe Fourierentwicklung für $R=R_{15}^{+}(1)$ 


\begin{tabular}{|c|c|c|c|c|c|}
\hline$n$ & $A(n)$ wie $\left(4.1^{\prime \prime \prime}\right)$ & $n$ & $B(n)$ wie $\left(4.2^{\prime \prime \prime}\right)$ & $p$ & $c(p)$ wie $(3.1)$ \\
\hline-1 & 1.00000000 & $-1-2 \omega$ & -1.08140355 & 1 & 1.000000000000000000 \\
\hline-2 & -0.84879187 & $-1-5 \omega$ & $-0.93138977-1.17755206 i$ & $2+\omega$ & 0.974455153242599482 \\
\hline $1+3 \omega$ & $0.50275275+0.04697536 i$ & $-4-8 \omega$ & 0.50678544 & 2 & -1.175006293091341487 \\
\hline-3 & 0.30114135 & $2-2 \omega$ & -0.37527418 & $3+2 \omega$ & 0.587080090761923263 \\
\hline$+3 \omega$ & $-0.25649097-0.62685123 i$ & $2-5 \omega$ & $-0.10486069-0.16029997 i$ & $3+\omega$ & -0.731596565810402583 \\
\hline$-1+3 \omega$ & $0.95817936+0.61535500 i$ & $-1-8 \omega$ & $0.01174434+0.24579559 i$ & $4+3 \omega$ & -0.647611327465178281 \\
\hline-4 & 0.09067399 & $-4-11 \omega$ & $-0.34838890+0.44307296 i$ & $4+\omega$ & -1.063828009303246491 \\
\hline$-2+3 \omega$ & $-0.09661951-0.29374428 i$ & $2-8 \omega$ & $-0.18191503+0.02758516 i$ & $5+3 \omega$ & -0.171927985296005837 \\
\hline-5 & -0.0050329 & $-7-14 \omega$ & -0.32510971 & $5+2 \omega$ & 0.162243908973960715 \\
\hline $3+6 \omega$ & 0.1806100 & $5-5 \omega$ & -0.14241592 & 5 & 0.463909856599805109 \\
\hline$-3+3 \omega$ & $-0.4789759-0.0638732 i$ & $-1-11 \omega$ & $-0.48312520+0.54884271 i$ & $6+5 \omega$ & -0.492467664482579292 \\
\hline $2+6 \omega$ & $0.1796944+0.1238969 i$ & $-4-14 \omega$ & $0.18259108+0.00945819 i$ & $6+\omega$ & -1.037948840088401175 \\
\hline $1+6 \omega$ & $-0.1475546+0.3665440 i$ & $5-8 \omega$ & $0.06416296+0.45787263 i$ & $7+4 \omega$ & -0.161982361949622732 \\
\hline-6 & -0.0521239 & $2-11 \omega$ & $-0.20394989+0.22208961 i$ & $7+3 \omega$ & 0.473628076670323690 \\
\hline$+6 \omega$ & $-0.0846500-0.1657513 i$ & $-7-17 \omega$ & $-0.12514586-0.24314596 i$ & $7+6 \omega$ & -0.163180256243435556 \\
\hline$-4+3 \omega$ & $-0.2821564+0.1734436 i$ & $-1-14 \omega$ & $-0.53834503+0.15742536 i$ & $7+\omega$ & -0.679178644943579004 \\
\hline$-1+6 \omega$ & $0.0570207+0.0333355 i$ & $-4-17 \omega$ & $-0.18464109+0.19646047 i$ & $9+5 \omega$ & 0.213111184872026280 \\
\hline-7 & -0.2880501 & $5-11 \omega$ & $-0.25503505+0.23763842 i$ & $9+4 \omega$ & 0.167705141445407517 \\
\hline$-5+3 \omega$ & $-0.1363293-0.0400874 i$ & $8-8 \omega$ & 0.2057412 & $9+7 \omega$ & -0.121364972205079390 \\
\hline$-2+6 \omega$ & $0.5479883+0.0719770 i$ & $-10-20 \omega$ & 0.1748470 & $9+2 \omega$ & -0.402412369132929498 \\
\hline $4+9 \omega$ & $0.234820-0.034015 i$ & $2-14 \omega$ & $0.3042652+0.0446103 i$ & $9+8 \omega$ & -0.267309615803910291 \\
\hline$-6+3 \omega$ & $-0.265490-0.073314 i$ & $-7-20 \omega$ & $-0.3751844-0.0613350 i$ & $9+\omega$ & 0.288920321792852548 \\
\hline$-3+6 \omega$ & $0.185229-0.271863 i$ & $-1-17 \omega$ & $-0.4674511-0.0615802 i$ & $10+7 \omega$ & -1.148463221948321295 \\
\hline $3+9 \omega$ & $0.031155-0.111214 i$ & $-4-20 \omega$ & $-0.0098086-0.1811271 i$ & $10+3 \omega$ & -0.164387727466458335 \\
\hline-8 & -0.040777 & $8-11 \omega$ & $0.2711391+0.1337722 i$ & $11+8 \omega$ & -0.571100613671304723 \\
\hline $2+9 \omega$ & $-0.202806-0.154279 i$ & $5-14 \omega$ & $-0.1213235+0.1673119 i$ & $11+3 \omega$ & -0.365805987476726974 \\
\hline $1+9 \omega$ & $0.072398+0.091894 i$ & $2-17 \omega$ & $0.1003287-0.0546814 i$ & $11+9 \omega$ & 0.796582846864461617 \\
\hline$-4+6 \omega$ & $-0.073717+0.085300 i$ & $-10-23 \omega$ & $-0.060670-0.078964 i$ & $11+2 \omega$ & 0.689383912996105084 \\
\hline$-7+3 \omega$ & $-0.04955+0.01585 i$ & $-1-20 \omega$ & $-0.052023-0.060898 i$ & $12+7 \omega$ & 0.143193304870476034 \\
\hline-9 & 0.03361 & $-7-23 \omega$ & $0.076165-0.188249 i$ & $12+5 \omega$ & 0.488137746036444524 \\
\hline$+9 \omega$ & $-0.27477+0.39673 i$ & $8-14 \omega$ & $-0.080109-0.020199 i$ & 11 & -0.400259936578718722 \\
\hline$-1+9 \omega$ & $-0.15750-0.05850 i$ & $11-11 \omega$ & -0.090937 & $13+7 \omega$ & 1.011333545701487928 \\
\hline$-5+6 \omega$ & $0.19262+0.05826 i$ & $-4-23 \omega$ & $0.102906-0.142757 i$ & $13+6 \omega$ & 0.429058923187070027 \\
\hline$-8+3 \omega$ & $0.2387+0.1579 i$ & $5-17 \omega$ & $0.102917+0.330327 i$ & $13+10 \omega$ & 1.133138316473920832 \\
\hline-10 & -0.1504 & $-13-26 \omega$ & 0.004191 & $13+3 \omega$ & 0.592652032294269351 \\
\hline$-2+9 \omega$ & $0.0372-0.1055 i$ & $-10-26 \omega$ & $0.063017+0.245977 i$ & $14+9 \omega$ & 0.356660081214303679 \\
\hline$-6+6 \omega$ & $0.1861+0.0567 i$ & $2-20 \omega$ & $0.102544+0.004009 i$ & $14+5 \omega$ & 1.147176248977683379 \\
\hline $6+12 \omega$ & -0.1223 & $-7-26 \omega$ & $-0.046168+0.026078 i$ & $13+12 \omega$ & 0.615044030387230990 \\
\hline $5+12 \omega$ & $-0.0287-0.0259 i$ & $-1-23 \omega$ & $-0.17444+0.04440 i$ & $13+\omega$ & -0.008354141622158139 \\
\hline $4+12 \omega$ & $0.0759-0.2055 i$ & $11-14 \omega$ & $0.05127+0.06245 i$ & $14+11 \omega$ & -0.237701658270889518 \\
\hline$-9+3 \omega$ & $0.1507-0.1108 i$ & $8-17 \omega$ & $0.08384-0.06709 i$ & $14+3 \omega$ & 0.140129666902732691 \\
\hline$-3+9 \omega$ & $0.0967-0.1931 i$ & $5-20 \omega$ & $-0.28396-0.06482 i$ & $15+11 \omega$ & -0.647939068747701437 \\
\hline $3+12 \omega$ & $0.070+0.058 i$ & $-13-29 \omega$ & $-0.13791+0.03030 i$ & $15+4 \omega$ & 0.133462415796061134 \\
\hline-11 & 0.053 & $-4-26 \omega$ & $-0.01300+0.12861 i$ & $16+9 \omega$ & 0.516426988448303458 \\
\hline $2+12 \omega$ & $-0.043-0.091 i$ & $2-23 \omega$ & $-0.08003-0.17634 i$ & $16+7 \omega$ & 0.157244674380075979 \\
\hline$-7+6 \omega$ & $0.226+0.031 i$ & $-10-29 \omega$ & $0.12098+0.24214 i$ & $15+13 \omega$ & 0.567307922226467313 \\
\hline $1+12 \omega$ & $0.166+0.123 i$ & $-7-29 \omega$ & $0.14348-0.03370 i$ & $15+2 \omega$ & 0.655717068568996562 \\
\hline$-4+9 \omega$ & $0.166-0.053 i$ & $-1-26 \omega$ & $-0.13990+0.14073 i$ & $15+14 \omega$ & 1.253025582852597562 \\
\hline$-10+3 \omega$ & $0.084-0.138 i$ & $11-17 \omega$ & $-0.01981+0.08583 i$ & $15+\omega$ & 1.089724039351992119 \\
\hline-12 & 0.008 & $14-14 \omega$ & 0.17653 & $17+11 \omega$ & 0.310059537326260739 \\
\hline$+12 \omega$ & $-0.146-0.073 i$ & $8-20 \omega$ & $0.11063+0.06194 i$ & $17+6 \omega$ & 0.055818843662576444 \\
\hline$-8+6 \omega$ & $0.065-0.129 i$ & $-16-32 \omega$ & 0.08890 & $17+12 \omega$ & 0.505609603901547895 \\
\hline$-5+9 \omega$ & $-0.048+0.158 i$ & $5-23 \omega$ & $0.21871-0.08937 i$ & $17+5 \omega$ & 0.906460595589592839 \\
\hline$-1+12 \omega$ & $-0.14+0.19 i$ & $-13-32 \omega$ & $-0.22309-0.02126 i$ & $16+15 \omega$ & 0.634707757489658556 \\
\hline$-11+3 \omega$ & $0.09+0.03 i$ & $-4-29 \omega$ & $-0.15793-0.11480 i$ & $16+\omega$ & -0.324366296855912582 \\
\hline
\end{tabular}

Tabelle 5.5: Metaplektische und automorphe Fourierentwicklung für $R=R_{15}^{+}(2)$ 


\subsection{Beobachtungen}

In [21] hat Waldspurger einen direkten Zusammenhang zwischen Eigenformen $f$ vom Gewicht $2 k$ und den nach Shimura korrespondierenden Eigenformen $g(z)=\sum c(n) e(n z)$ zum Gewicht $k+1 / 2$ erwiesen: Für quadratfreies $n$ ist $c^{2}(n)$ im wesentlichen proportional zu dem Wert der um den Charakter des quadratischen Körpers getwisteten Reihe $L_{f}(s, \chi)$ an der Stelle $s=k$.

Mit den Daten, die sowohl zu den automorphen, als auch zu den kubisch korrespondierenden Formen gesammelt worden sind, war es möglich, analoge Vermutungen auch hier zu prüfen. Allerdings konnten keine zum quadratischen Fall vergleichbaren Zusammenhänge gefunden werden.

Um die erlangten numerischen Ergebnisse zur Bestimmung von $L$-Reihen für Spitzenformen bei $s=1 / 2 \mathrm{zu}$ verwenden, ist es nötig, eine schnell konvergierende Darstellung für diese Reihen zu finden. Die einfache Darstellung

$$
L_{f}(s, \chi)=\frac{1}{6} \sum c(\mu) N(\mu)^{-s}
$$

ist dafür keinesfalls geeignet, wenn man das langsame Wachstum der $N(\mu)$ bedenkt. Für die letzte Primstelle der Tabelle gilt $N(16+\omega)^{-1 / 2} \approx 0.06$, so daß die Summation der ersten $161 \mu$, welche sich mit den angegebenen $c(p)$ für die ersten 54 Primzahlen bestimmen lassen, noch nicht einmal die erste Stelle von $L_{f}(1 / 2, \chi)$ sicher bestimmen. Es ist auch nicht sofort klar, daß $s=1 / 2$ im Konvergenzgebiet von (5.1) liegt.

Eine günstigere Darstellung zur Bestimmung von $L_{f}(s, \chi)$ ist

$$
\begin{aligned}
& \frac{3^{s+1}}{2} \tau(\bar{\chi})(2 \pi)^{-2 s} \Gamma\left(s+\frac{i R}{2}\right) \Gamma\left(s-\frac{i R}{2}\right) L_{f}(s, \chi)= \\
& \tau(\bar{\chi}) \sum_{\mu \in \lambda^{-1} \mathbf{Z}[\omega]} c(\mu) \chi(\lambda \mu) N(\mu)^{-s} \int_{\substack{|\mu| \\
|M|}}^{\infty} K_{i R}(4 \pi v) v^{2 s-1} d v+ \\
& +\tau(\chi)|M|^{1-2 s} \sum_{\mu \in \lambda^{-1} \mathbf{Z}[\omega]} c(\mu) \bar{\chi}(\lambda \mu) N(\mu)^{-s} \int_{\frac{|\mu|}{|M|}}^{\infty} K_{i R}(4 \pi v) v^{1-2 s} d v
\end{aligned}
$$

Die folgenden Ausführungen zu der Gültigkeit von (5.2) folgen einer persönlichen Mitteilung von Herrn Professor S. J. Patterson [9]. 
Sei wie in Kapitel 3 eine Spitzenform $f$ auf $\mathbb{H}^{3}$ gegeben, invariant unter $\Gamma$ mit

$$
\begin{aligned}
f(z, v) & =\sum_{\mu \in \mathbf{Z}[\omega]} c(\mu) v K_{i R}\left(\frac{2 \pi}{A}|\mu| v\right) e\left(\frac{\langle\mu, z\rangle}{A}\right) \\
& =\sum_{\mu \in \frac{1}{\lambda} \mathbf{Z}[\omega]} c(\lambda \mu) v K_{i R}(4 \pi|\mu| v) e(2\langle\mu, z\rangle),
\end{aligned}
$$

wobei $\lambda=\sqrt{-3}=\omega-\omega^{2}$. Sei weiter $f$ gerade, dann gilt $c(\varepsilon \mu)=c(\mu)$ für alle Einheiten $\varepsilon$, und sei $f$ eine normierte Eigenform der Hecke-Operatoren mit $c(1)=1$.

Für einen primitiven Charakter $\chi$ von Modulus $M$ kann man $\tau(\chi)$ und $f_{\chi}$ durch

$$
\tau(\chi)=\sum_{j(M)} \chi(j) e\left(\frac{2 j}{\lambda M}\right)
$$

und

$$
f_{\chi}(z, v)=\sum \overline{\chi(j)} f\left(z+\frac{j}{M}, v\right)=\tau(\chi) \sum_{\mu \in \frac{1}{\lambda} \mathbf{Z}[\omega]} c(\lambda \mu) \chi(\lambda \mu) K_{i R}(4 \pi|\mu| v) e(2\langle\mu, z\rangle)
$$

definieren. Sei weiter $\chi(-1)=1$ und $\chi(\omega)=1$. Mit

$$
\int_{0}^{\infty} K_{i R}(4 \pi v) v^{2 s-1} d v=\frac{1}{4}(2 \pi)^{-2 s} \Gamma\left(s+\frac{i R}{2}\right) \Gamma\left(s-\frac{i R}{2}\right)
$$

erhält man

$$
\int_{0}^{\infty} f_{\chi}(0, v) v^{2 s-2} d v=6 \tau(\bar{\chi}) \frac{3^{s}}{4}(2 \pi)^{-2 s} \Gamma\left(s+\frac{i R}{2}\right) \Gamma\left(s-\frac{i R}{2}\right) L_{f}(s, \chi)
$$

wobei

$$
L_{f}(s, \chi)=\prod_{\pi}\left(1-\chi(\pi) c\left(\lambda^{-1} \pi\right) N(\pi)^{-s}+\chi(\pi)^{2} N(\pi)^{-2 s}\right)^{-1}=\sum_{\mu \in \mathbf{Z}[\omega] /(-\omega)}^{\prime} c(\mu) \chi(\mu) N(\mu)^{-s} .
$$

Außerdem ist

$$
\begin{aligned}
f_{\bar{\chi}}\left(\left(\begin{array}{cc}
0 & -1 / M \\
M & 0
\end{array}\right)(z, v)\right) & =\sum_{j(M)} f\left(\frac{j}{M}+\left(\begin{array}{cc}
0 & -1 / M \\
M & 0
\end{array}\right)(z, v)\right) \bar{\chi}(j) \\
& =\sum_{j(M)} \bar{\chi}(j) f\left(\left(\begin{array}{cc}
1 & j / M \\
0 & 1
\end{array}\right)\left(\begin{array}{cc}
0 & -1 / M \\
M & 0
\end{array}\right)(z, v)\right) \\
& =\sum_{j(M)} \bar{\chi}(j) f\left(\left(\begin{array}{cc}
j & -1 / M \\
M & 0
\end{array}\right)(z, v)\right) .
\end{aligned}
$$


Mit $\bar{j}$ so gewählt, daß $j \bar{j} \equiv 1(\bmod M)$, gilt $\left(\begin{array}{cc}j & -1 / M \\ M & 0\end{array}\right)\left(\begin{array}{cc}1 & \bar{j} / M \\ 0 & 1\end{array}\right)=\left(\begin{array}{c}j \\ M\end{array}\left(\begin{array}{l}j \bar{j}-1) / M \\ \bar{j}\end{array}\right) \in S L_{2}(\mathbf{Z}[\omega])\right.$. Damit vereinfacht sich (5.3) zu

$$
\begin{aligned}
f_{\bar{\chi}}\left(\left(\begin{array}{cc}
0 & -1 / M \\
M & 0
\end{array}\right)(z, v)\right) & =\sum_{j(M)} \bar{\chi}(j) f\left(\left(\begin{array}{cc}
1 & -\bar{j} / M \\
0 & 1
\end{array}\right)(z, v)\right) \\
& =\chi(-1) f_{\chi}(z, v) \\
& =f_{\chi}(z, v) .
\end{aligned}
$$

Daher gilt

$$
\int_{0}^{\infty} f_{\chi}(0, v) v^{2 s-2} d v=\frac{3^{s+1}}{2} \tau(\bar{\chi})(2 \pi)^{-2 s} \Gamma\left(s+\frac{i R}{2}\right) \Gamma\left(s-\frac{i R}{2}\right) L_{f}(s, \chi)
$$

und außerdem

$$
\begin{aligned}
\int_{0}^{\infty} f_{\chi}(0, v) v^{2 s-2} d v & =\int_{0}^{|M|^{-1}} f_{\chi}(0, v) v^{2 s-2} d v+\int_{|M|^{-1}}^{\infty} f_{\chi}(0, v) v^{2 s-2} d v \\
& =\int_{0}^{|M|^{-1}} f_{\bar{\chi}}\left(0, \frac{1}{|M|^{2} v}\right) v^{2 s-2} d v+\int_{|M|^{-1}}^{\infty} f_{\chi}(0, v) v^{2 s-2} d v \\
& =\int_{|M|^{-1}}^{\infty} f_{\bar{\chi}}(0, v) v^{-2 s} d v|M|^{1-2 s}+\int_{|M|^{-1}}^{\infty} f_{\chi}(0, v) v^{2 s-2} d v
\end{aligned}
$$

Mit

$$
\begin{aligned}
\int_{|M|^{-1}}^{\infty} f_{\chi}(0, v) v^{2 s-2} d v & =\int_{|M|^{-1}}^{\infty} \tau(\bar{\chi}) \sum c(\lambda \mu) \chi(\lambda \mu) v K_{i R}(4 \pi|\mu| v) v^{2 s-2} d v \\
& =\tau(\bar{\chi}) \sum c(\lambda \mu) \chi(\lambda \mu) N(\mu)^{-s} \int_{\frac{|\mu|}{|M|}}^{\infty} K_{i R}(4 \pi v) v^{2 s-1} d v
\end{aligned}
$$

und

$$
\int_{|M|^{-1}}^{\infty} f_{\bar{\chi}}(0, v) v^{-2 s} d v=\tau(\chi) \sum c(\mu) \bar{\chi}(\lambda \mu) N(\mu)^{-s} \int_{\frac{|\mu|}{|M|}}^{\infty} K_{i R}(4 \pi v) v^{1-2 s} d v
$$

folgt die Gültigkeit von (5.2). Anzumerken ist noch, daß für $s=1 / 2$

$$
\Gamma\left(1 / 2+\frac{i R}{2}\right) \Gamma\left(1 / 2-\frac{i R}{2}\right)=\frac{\pi}{\cosh \left(\frac{\pi}{2} R\right)}
$$


gilt. Damit vereinfacht sich (5.2) zu

$$
L_{f}\left(\frac{1}{2}, \chi\right)=\frac{1}{\tau(\bar{\chi})} \frac{8 \cosh \left(\frac{\pi}{2} R\right)}{3 \sqrt{3}} \operatorname{Re}\left(\tau(\chi) \sum_{\mu \in \lambda^{-1} \mathbf{Z}[\omega]} c(\lambda \mu) \bar{\chi}(\lambda \mu) N(\mu)^{-1 / 2} \int_{\frac{|\mu|}{|M|}}^{\infty} K_{i R}(4 \pi v) d v\right)
$$

da $\tau(\bar{\chi})=\overline{\tau(\chi)}$ gilt.

Ein vergleichbares Verhalten zum quadratischen von Waldspurger untersuchten Fall wäre eine Proportionalität zwischen $L_{f}\left(\frac{1}{2}, \chi\right)$ und $A^{3}(n)$. Die einfachste Möglichkeit (5.5) anzuwenden, ist es, das Argument von dieser Gleichung zu betrachten. Da alle Terme bis auf $L_{f}\left(\frac{1}{2}, \chi\right)$ und $\frac{1}{\tau(\bar{\chi})}$ reell sind, müßte im Falle einer Proportionalität auch

$$
3 \arg (A(n))+\arg (\tau(\bar{\chi}))=3 \arg (A(n))-\arg (\tau(\chi))=\left\{\begin{array}{l}
0 \\
\pi
\end{array}\right.
$$

gelten, und hierfür ist keine Auswertung von $L_{f}(s, \chi)$ nötig. Mögliche Kandidaten für $\chi$ sind etwa für $n=n_{1}+3 n_{2} \omega$ quadratfrei:

$$
\chi: m \mapsto\left(\begin{array}{c}
\lambda^{a} \omega^{b} m \\
n
\end{array}\right)_{3} \quad \text { für verschiedene } 0 \leq a, b \leq 2 .
$$

Die Abbildungen 5.7 und 5.8 zeigen auf der $x$-Achse das Argument von $1 / \tau(\bar{\chi})$ und auf der $y$-Achse das Argument von $A(n)$ für verschiedene $n, a$ und $b$ für $R=R_{12}^{+}$bzw. $R=R_{8}^{-}$. Vergleichbare Plots finden sich für andere Eigenformen auf der CD im Verzeichnis DOC und die dazu gehörenden Daten für die Plots im Verzeichnis GNUPLOT. In keinem dieser Plots ist ein proportionaler Zusammenhang zu erkennen.

Da im quadratischen Fall die $c(n)$ reell sind und das Vorzeichen der $c(n)$ noch nicht verstanden ist, könnte man vermuten, daß in diesem kubischen Fall nicht das Argument, wohl aber der Absolutbetrag einem Zusammenhang genügt. Ein solcher Zusammenhang ist etwas schwerer zu prüfen, da dies in (5.5) ein Auswerten der Integrale über K-Bessel Funktionen und eine Konvergenz der Reihe erfordert. Dieser Zusammenhang wird in den Tabellen 5.6-5.8 exemplarisch dargestellt; die entsprechenden Tabellen für andere Werte von $a, b$ und $R$ finden sich auch auf der CD im Verzeichnis DOC. Auch hier ist für keine Werte von $R$ eine Proportionalität festzustellen. 


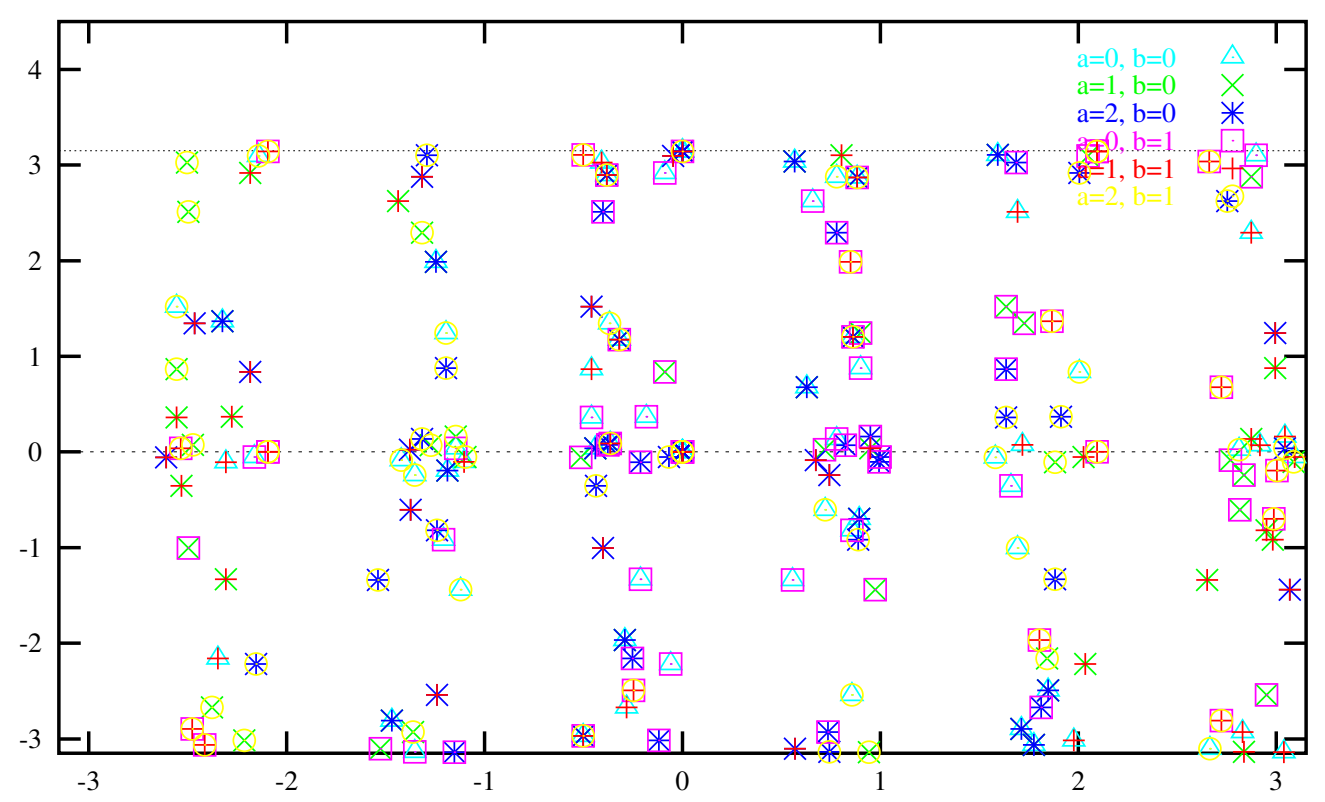

Abbildung 5.7: Plot der Argumente bei $R=R_{12}^{+}$für quadratfreie $n$ und $3 \nmid n$

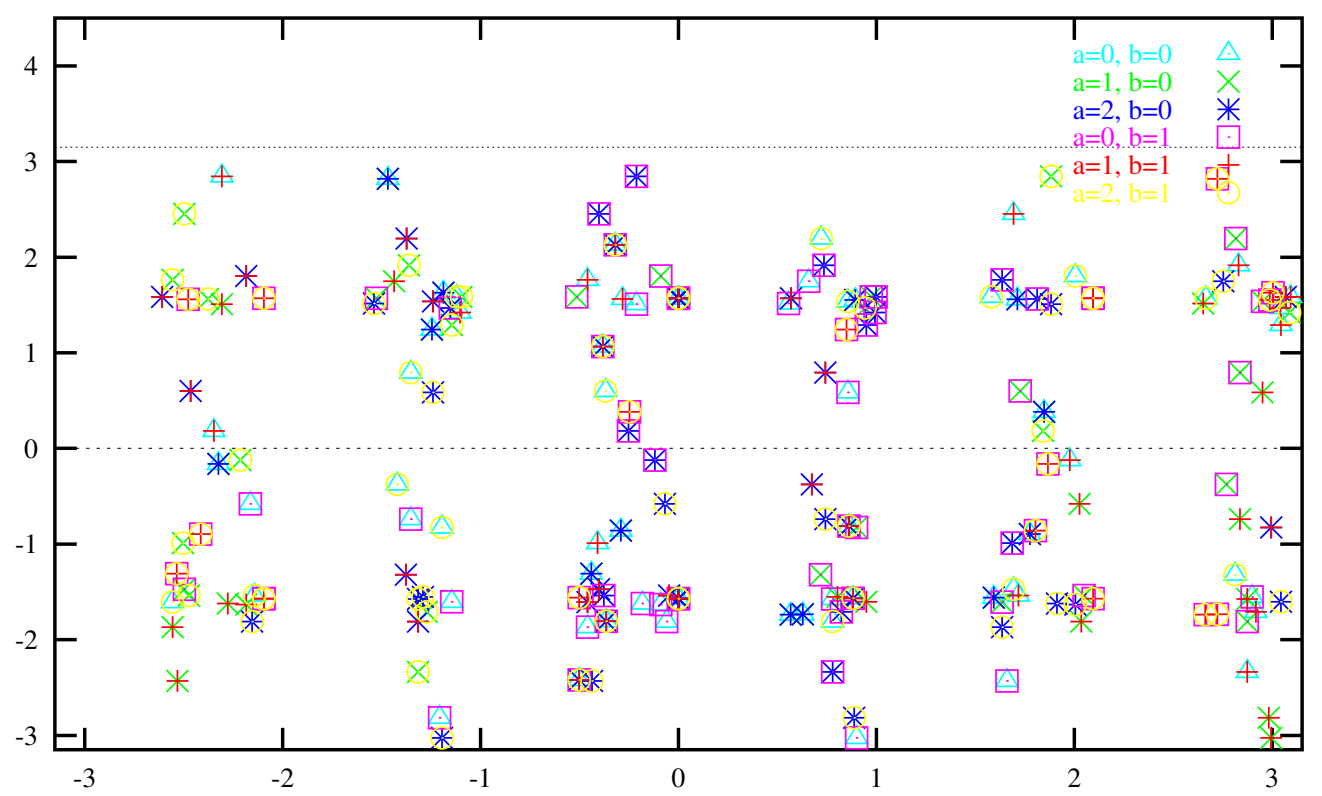

Abbildung 5.8: Plot der Argumente bei $R=R_{8}^{-}$für quadratfreie $n$ und $3 \nmid n$ 


\begin{tabular}{|c|c|c|r|}
\hline$n$ & $L_{f}(1 / 2, \chi)$ & $\left|L_{f}(1 / 2, \chi) / A^{3}(n)\right|$ & $\arg \left(L_{f}(1 / 2, \chi) / A^{3}(n)\right)$ \\
\hline-2 & 0.337253 & 0.181279 & 0.0 \\
$3 \omega-2$ & $-0.028238-0.0187834 * I$ & 0.0643731 & 0.608042 \\
$3 \omega-4$ & $0.126652+0.125038 * I$ & 0.64056 & 0.50297 \\
$3 \omega-5$ & $-0.0340537+0.00735992 * I$ & 0.668868 & 1.22402 \\
-5 & 0.764345 & 8.12389 & 0.0 \\
$6 \omega-4$ & $0.0235182-0.0116586 * I$ & 0.215818 & 2.7486 \\
$6 \omega-5$ & $-0.0228923+0.18598 * I$ & 49.0173 & 1.60094 \\
$3 \omega-7$ & $-0.00592294-0.00746531 * I$ & 0.179742 & 1.08657 \\
\hline
\end{tabular}

Tabelle 5.6: $A^{3}(n)$ und $L_{f}(s, \chi)$ für $a=0, b=0$ und $R=R_{1}^{+}$

\begin{tabular}{|c|c|c|r|}
\hline$n$ & $L_{f}(1 / 2, \chi)$ & $\left|L_{f}(1 / 2, \chi) / A^{3}(n)\right|$ & $\arg \left(L_{f}(1 / 2, \chi) / A^{3}(n)\right)$ \\
\hline-2 & 0.346876 & 0.617743 & 1.5708 \\
$3 \omega-2$ & $-0.0174655-0.0116177 * I$ & 2.03675 & -0.579393 \\
$3 \omega-4$ & $0.120612+0.119075 * I$ & 2.10863 & -2.45886 \\
$3 \omega-5$ & $-0.0470961+0.0101787 * I$ & 0.597969 & -2.16484 \\
-5 & 0.7034 & 143.745 & 1.5708 \\
$6 \omega-4$ & $0.00565533-0.0028035 * I$ & 0.789358 & 2.53388 \\
$6 \omega-5$ & $-0.0211033+0.171446 * I$ & 417.152 & -2.80075 \\
$3 \omega-7$ & $0.00562118+0.00708496 * I$ & 8.18148 & -1.38201 \\
\hline
\end{tabular}

Tabelle 5.7: $A^{3}(n)$ und $L_{f}(s, \chi)$ für $a=0, b=0$ und $R=R_{2}^{+}$

\begin{tabular}{|c|c|c|r|}
\hline$n$ & $L_{f}(1 / 2, \chi)$ & $\left|L_{f}(1 / 2, \chi) / A^{3}(n)\right|$ & $\arg \left(L_{f}(1 / 2, \chi) / A^{3}(n)\right)$ \\
\hline-2 & 0.230279 & 39.9072 & 1.5708 \\
$3 \omega-2$ & $0.111542+0.0741955 * I$ & 17.2076 & -0.706227 \\
$3 \omega-4$ & $0.0304893+0.0301008 * I$ & 1.27753 & 0.992207 \\
$3 \omega-5$ & $0.085167-0.0184069 * I$ & 2.04333 & 0.835204 \\
-5 & 0.182509 & 198.847 & 1.5708 \\
$6 \omega-4$ & $0.154005-0.0763443 * I$ & 45.3757 & 0.0217087 \\
$6 \omega-5$ & $0.00128856-0.0104684 * I$ & 18.6014 & 2.63334 \\
$3 \omega-7$ & $-0.000286623-0.000361261 * I$ & 0.0444567 & -3.11699 \\
\hline
\end{tabular}

Tabelle 5.8: $A^{3}(n)$ und $L_{f}(s, \chi)$ für $a=0, b=0$ und $R=R_{1}^{-}$ 


\section{Kapitel 6}

\section{Numerischer Anhang}

In diesem Anhang sollen einige Erklärungen zu den verwendeten numerischen Methoden gegeben werden. Nur nicht offensichtliche Vorgehensweisen sollen dabei berücksichtigt werden. Die angestrebte Genauigkeit in den ersten beiden Abschnitten beträgt 18 Stellen.

\subsection{Besselfunktionen}

Zur Berechnung der metaplektischen Funktionen werden die modifizierten Besselfunktionen $K_{\nu}(x)$ benötigt. Dabei ist $x$ eine beliebige positive reelle Zahl und $\nu$ komplex mit $0<\operatorname{Im} \nu<25$ und $\operatorname{Re} \nu=0,1$. Zur Berechnung wird für große $x$ die asymptotische Reihe

$$
K_{\nu}(x)=\sqrt{\frac{\pi}{2 x}} e^{-x} \sum_{n=1} d_{n} ; \quad d_{0}=1, d_{n}=\frac{4 \nu^{2}-(2 n-1)^{2}}{8 n x} d_{n-1}
$$

und für die übrigen $x$ die Potenzreihe

$$
\begin{aligned}
K_{\nu}(x) & =\frac{\pi}{2 \sin \pi \nu}\left(I_{-\nu}(x)-I_{\nu}(x)\right) \text { mit } \\
I_{\nu}(x) & =\sum_{n=0} d_{n} \quad d_{0}=\frac{1}{\Gamma(\nu+1)}\left(\frac{x}{2}\right)^{\nu}, d_{n}=\frac{x^{2}}{4 n(\nu+n)} d_{n-1}
\end{aligned}
$$

verwendet. Während $K_{\nu}(x)$, etwa nach (6.1), für große $x$ wie $e^{-x}$ abklingt, wächst $I_{\nu}(x)$ exponentiell wie $e^{x}$. Dementsprechend tritt in (6.2) ein großer Stellenverlust auf. Zur Berechnung von $K_{\nu}(x)$ nach (6.2) wurde daher ein $\mathrm{C}++$-Paket mit multipler Genauigkeit verwendet. Es zeigt sich, daß 50 Dezimalstellen ausreichend sind und man dann einen ausreichend großen Bereich erhält, in dem beide Darstellungen gültig sind. Einige Versuche zeigen, daß man für $x>30+2|\nu|$ 
die Darstellung (6.1) und für $x \leq 30+2|\nu|$ die Darstellung (6.2) wählen kann. Dabei sind höchstens 60 Terme in (6.1) und 160 Terme in (6.2) ausreichend. Zur Berechnung von (6.2) benötigt man auch noch die Gammafunktion $\Gamma(\nu)$ für komplexe $\nu$ auf 50 Stellen Genauigkeit.

Zur Berechnung der Gammafunktion $\Gamma(\nu)$ wurde die Stirlingsche Formel verwendet. Einige numerische Beispiele zeigen, daß das folgende Verfahren gute Ergebnisse liefert. Man addiert zunächst zu $\nu$ eine ganze Zahl $n$, so daß $\operatorname{Re}(\nu+n)>25$ ist. Dann berechnet man 30 Terme der Stirlingschen Formel auf 50 Stellen und verwendet die Funktionalgleichung, um aus $\Gamma(\nu+n)$ dann $\Gamma(\nu)$ zu berechnen.

\subsection{Integral der Besselfunktion}

Für die Berechnungen in Kapitel 5 wurde noch das Integral der Besselfunktion

$$
I K_{\nu}(x)=\int_{x}^{\infty} K_{\nu}(\xi) d \xi \quad \text { mit } \quad I K_{\nu}(0)=\frac{\pi}{2 \cosh \frac{\pi \nu}{2 i}}
$$

benötigt. Es erscheint naheliegend, in Analogie zu Abschnitt 6.1, aus den Gleichungen (6.1) und (6.2) durch Integration eine asymptotische Reihe und eine Potenzreihe herzuleiten. Dabei zeigt sich allerdings, daß die so gewonnenen Reihen für die interessierenden $\nu$-Werte keinen Überlappungsbereich mit Gültigkeit beider Darstellungen besitzen. Stattdessen wurde die Potenzreihe

$$
\begin{aligned}
I K_{\nu}(x) & =I K_{\nu}(0)+\frac{\pi}{2 \sin \pi \nu}\left(I I_{-\nu}(x)-I I_{\nu}(x)\right) \mathrm{mit} \\
I I_{\nu}(x) & =\sum_{n=0} \frac{d_{n}}{(2 n+\nu+1)} \quad d_{0}=\frac{1}{\Gamma(\nu+2)}\left(\frac{x}{2}\right)^{\nu+1}, d_{n}=\frac{x^{2}}{4 n(\nu+n)} d_{n-1}
\end{aligned}
$$

ausgewertet. Anstelle der asymptotischen Reihe wurde für große $x$ die Darstellung

$$
I K_{\nu}(x)=-(\nu-1) x K_{\nu}(x) S_{-1, \nu-1}(i x)+i x K_{\nu-1)}(x) S_{0, \nu}(i x)
$$

aus [7] ausgewertet, wobei $S_{\mu, \nu}(x)$ die Lommelfunktionen sind. Zur Berechnung der $S_{\mu, \nu}(x)$ kann man die asymptotische Darstellung

$$
S_{\mu, \nu}(x)=\sum_{n=0} d_{n} \quad d_{0}=x^{\mu-1}, d_{n}=-\frac{(\mu-2 n+1)^{2}-\nu^{2}}{x^{2}} d_{n-1}
$$

verwenden und erhält dann ein ausreichendes Überlappungsgebiet um $x \approx 47+\operatorname{Im} \nu$. Dann zeigt sich, daß 60 Terme in der asymptotischen Reihe und 160 Terme in der Potenzreihe in jedem Fall ausreichen. 


\subsection{Kubisches Restsymbol}

Die Berechnung des kubischen Restsymbols $\left(\begin{array}{l}k \\ n\end{array}\right)_{3}$ kann für die benötigten Aufgaben auf Fälle mit $N(n) \nmid 3$, und $n \equiv \pm 1 \quad(\bmod 3)$ beschränkt werden. Dann kann mit einer Primfaktorzerlegung von $n= \pm \prod_{i=1}^{m} p_{i}^{a_{i}}$ das Restsymbol durch

$$
\left(\begin{array}{l}
k \\
n
\end{array}\right)_{3}=\prod_{i=1}^{m}\left(\begin{array}{c}
k \\
p_{i}^{a_{i}}
\end{array}\right)_{3}
$$

bestimmt werden, wobei die $p$ alle der Bedingung $p \equiv-1(\bmod 3)$ genügen sollen. Mit dem Analogon zum kleinen Satz von Fermat gilt

$$
\left(\begin{array}{c}
k \\
p^{a}
\end{array}\right)_{3} \equiv k^{a \frac{N(p)-1}{3}}(\bmod \mathrm{p}) .
$$

Um dies durchführen zu können, muß die algebraische Primfaktorzerlegung von $n$ bestimmt werden.

Zur Zerlegung einer ganzen algebraischen Zahl $n$ in Primfaktoren kann zuerst die Norm $N(n)=\prod_{i=1}^{m} r_{i}^{a_{i}}$ in ein Produkt rationaler Primzahlen zerlegt werden. Dann können die rationalen Primzahlen $r$ mit $r \equiv 2(\bmod 3)$ direkt als Primfaktoren in $n$ mit der Vielfachheit $a / 2$ übernommen werden. Da $N(n) \nmid 3$ bleibt dann noch die Aufgabe, für die voll zerlegten rationalen Primzahlen deren algebraische Primfaktoren aus der Kenntnis ihrer Norm zu bestimmen. Das ist ein Problem aus dem Gebiet der binären Formen. Dazu kann man das in [17] beschriebene auf Lagrange zurückgehende Verfahren oder die auf Hermite zurückgehende, auf dem Euklidischen Algorithmus basierende Methode aus [3] benutzen. 


\section{Literaturverzeichnis}

[1] L. Bianchi, Geometrische Darstellung der Gruppen Linearer Substitutionen mit ganzen complexen Coefficienten nebst Anwendungen auf die Zahlentheorie, in: Math. Ann. 38 (1892), S. 313-333.

[2] F. Grunewald und W. Huntebrinker, A Numerical Study of Eigenvalues of the Hyperbolic Laplacian for Polyhedra with One Cusp, in: Exp. Math. 5 (1996), S. 57-80.

[3] K. Hardy, J. B. Muskat und K. S. Williams, A deterministic algorithm for solving $n=$ $f u^{2}+g v^{2}$ in coprime integers $u$ and $v$, in: Math. Comput. 55 (1990), S. 327-343.

[4] B. Harish-Chandra, Automorphic Forms on Semi-Simple Lie Groups. Springer Lecture Notes in Mathematics 62 (1968).

[5] K. Ireland und M. Rosen, A Classical Introduction to modern Number Theory. Springer Graduate texts in Mathematics 84 2. ed. New York u. a. (1990).

[6] W. Kohnen und D. Zagier, Values of $L$-Series of Modular Forms at the Center of the Critical Strip, in: Invent. math. 64 (1981), S. 175-198.

[7] Y. L. Luke, Integrals of Bessel functions. New York u. a. (1962).

[8] C. Batut, K. Belabas, D. Benardi, H. Cohen und M. Olivier, User's Guide to PARI-GP, durch anonymous ftp von ftp://megrez.math.u-bordeaux.fr/pub/pari (2002).

[9] S. J. Patterson, persönliche Mitteilung (2002).

[10] S. J. Patterson, A cubic analogue of the theta series, in: J. reine angew. Math. 296 (1977), S. $125-161$. 
[11] S. J. Patterson, The Cubic Shimura Correspondence, in: Asian. J. Math., Vol. 2, No. 4 (1998), S. 957-982.

[12] S. J. Patterson, The level structure of exceptional metaplectic representations, in: J. reine angew. Math. 494 (1998), S. 85-100.

[13] H. Poincaré, Mémoire sure les groupes kleinéens, in: Acta Mathematica 3 (1883), S. 49-92.

[14] W. H. Press, B. P. Flannery, S. A. Teukolsky, W. T. Vetterling, Numerical Recipes in C. Cambridge University Press (1988).

[15] N. Proskurin, Cubic metaplectic Forms and theta functions. Springer Lecture Notes in Mathematics 1677, Berlin u. a. (1998).

[16] P. Sarnak, Statistical Properties of eigenvalues of Hecke Operators, in: A. Adolphson, J. Conrey, A. Ghosh, R. Yager (Eds.), Analytic Number Theory and Diophantine Problems: Proceedings of a conference at Oklahoma State University (Progress in mathematics 70) Boston u. a. (1987), S. 321-331.

[17] A. Scholz, B. Schoeneberg, Einführung in die Zahlentheorie. Sammlung Göschen 5131, Berlin u. a. (1972).

[18] H. M. Stark, Fourier Coefficients of Maass Waveforms, in: R. A. Rankin (Ed.), Modular Forms. Symposium on modular forms of one and several variables Chichester (1984), S. 263269.

[19] G. Steil, Eigenvalues of the Laplacian for Bianchi Groups. Hamburger Beiträge zur Angewandten Mathematik, Reihe A, Preprint 119, Hamburg (1997).

[20] K. Stramm, Kleine Eigenwerte des Laplace-Operators zu Kongruenzgruppen. Schriftenreihe des Mathematischen Instituts und des Graduiertenkollgs der Universität Münster, 3. Ser. 11 Münster (1994).

[21] J. L. Waldspurger, La correspondence de Shimura, in: J. Math. pures et appl., 60 (1980), S. $1-132$. 